\title{
Evolutionary roads leading to low effective spins, high black hole masses, and 01/02 rates for LIGO/Virgo binary black holes ${ }^{\star}$
}

K. Belczynski ${ }^{1}$, J. Klencki ${ }^{2}$, C. E. Fields ${ }^{3}$, A. Olejak ${ }^{4,5}$, E. Berti ${ }^{6}$, G. Meynet ${ }^{7}$, C. L. Fryer ${ }^{8}$, D. E. Holz ${ }^{9}$, R. O’ Shaughnessy ${ }^{10}$, D. A. Brown ${ }^{11}$, T. Bulik ${ }^{4}$, S. C. Leung ${ }^{12,13}$, K. Nomoto ${ }^{12}$, P. Madau ${ }^{14}$, R. Hirschi ${ }^{15,12}$, E. Kaiser ${ }^{15}$, S. Jones ${ }^{8}$, S. Mondal $^{1}$, M. Chruslinska ${ }^{2}$, P. Drozda ${ }^{4}$, D. Gerosa ${ }^{16}$, Z. Doctor ${ }^{9}, 22$, M. Giersz ${ }^{1}$, S. Ekstrom ${ }^{7}$, C. Georgy ${ }^{7}$, A. Askar ${ }^{17}$, V. Baibhav ${ }^{6}$, D. Wysocki ${ }^{10}$, T. Natan ${ }^{18}$, W. M. Farr ${ }^{19}$, G. Wiktorowicz ${ }^{20}$, M. Coleman Miller ${ }^{21}$, B. Farr ${ }^{22}$, and J.-P. Lasota ${ }^{1,23}$

1 Nicolaus Copernicus Astronomical Center, Polish Academy of Sciences, ul. Bartycka 18, 00-716 Warsaw, Poland e-mail: chrisbelczynski@gmail.com

2 Department of Astrophysics/IMAPP, Radboud University, PO Box 9010, 6500 GL Nijmegen, The Netherlands

3 Department of Physics and Astronomy, Michigan State University, East Lansing, MI 48824, USA

4 Astronomical Observatory, Warsaw University, Al. Ujazdowskie 4, 00-478 Warsaw, Poland

5 Center for Theoretical Physics, Polish Academy of Sciences, Al. Lotnikow 32/46, 02-668 Warsaw, Poland

${ }^{6}$ Department of Physics and Astronomy, Johns Hopkins University, Baltimore, MD 21218, USA

7 Department of Astronomy, University of Geneva, Chemin des Maillettes 51, 1290 Versoix, Switzerland

8 CCS-2, MSD409, Los Alamos National Laboratory, Los Alamos, NM 87545, USA

9 University of Chicago, Chicago, IL 60637, USA

${ }^{10}$ Center for Computational Relativity and Gravitation, Rochester Institute of Technology, Rochester, NY 14623, USA

11 Department of Physics, Syracuse University, Syracuse, NY 13224, USA

12 Kavli Institute for the Physics and Mathematics of the Universe (WPI), The University of Tokyo, Kashiwa, Chiba 277-8583, Japan

13 TAPIR, Walter Burke Institute for Theoretical Physics, Mailcode 350-17, Caltech, Pasadena, CA 91125, USA

14 Department of Astronomy \& Astrophysics, University of California, 1156 High Street, Santa Cruz, CA 95064, USA

15 Lennard-Jones Laboratories, Keele University, Keele ST5 5BG, UK

16 School of Physics \& Astronomy \& Institute for Gravitational Wave Astronomy, University of Birmingham, Birmingham, UK

17 Lund Observatory, Department of Astronomy, and Theoretical Physics, Lund University, Box 43, 22100 Lund, Sweden

18 Physics Department, Kenyon College, 201 North College RD, Gambier, OH 43022, USA

19 Department of Physics and Astronomy, Stony Brook University, Stony Brook, NY 11794, USA

20 National Astronomical Observatories \& University of the Chinese Academy of Sciences, Beijing, PR China

21 Department of Astronomy and Joint Space-Science Institute University of Maryland, College Park, MD 20742-2421, USA

22 Department of Physics, University of Oregon, Eugene, OR 97403, USA

23 Institut d'Astrophysique de Paris, CNRS et Sorbonne Université, UMR 7095, 98bis Bd Arago, 75014 Paris, France

Received 20 August 2019 / Accepted 5 March 2020

\section{ABSTRACT}

All ten LIGO/Virgo binary black hole (BH-BH) coalescences reported following the O1/O2 runs have near-zero effective spins. There are only three potential explanations for this. If the BH spin magnitudes are large, then: (i) either both BH spin vectors must be nearly in the orbital plane or (ii) the spin angular momenta of the BHs must be oppositely directed and similar in magnitude. Then there is also the possibility that (iii) the BH spin magnitudes are small. We consider the third hypothesis within the framework of the classical isolated binary evolution scenario of the $\mathrm{BH}-\mathrm{BH}$ merger formation. We test three models of angular momentum transport in massive stars: a mildly efficient transport by meridional currents (as employed in the Geneva code), an efficient transport by the Tayler-Spruit magnetic dynamo (as implemented in the MESA code), and a very-efficient transport (as proposed by Fuller et al.) to calculate natal $\mathrm{BH}$ spins. We allow for binary evolution to increase the BH spins through accretion and account for the potential spin-up of stars through tidal interactions. Additionally, we update the calculations of the stellar-origin BH masses, including revisions to the history of star formation and to the chemical evolution across cosmic time. We find that we can simultaneously match the observed $\mathrm{BH}-\mathrm{BH}$ merger rate density and $\mathrm{BH}$ masses and $\mathrm{BH}-\mathrm{BH}$ effective spins. Models with efficient angular momentum transport are favored. The updated stellar-mass weighted gas-phase metallicity evolution now used in our models appears to be key for obtaining an improved reproduction of the LIGO/Virgo merger rate estimate. Mass losses during the pair-instability pulsation supernova phase are likely to be overestimated if the merger GW170729 hosts a BH more massive than $50 M_{\odot}$. We also estimate rates of black hole-neutron star (BH-NS) mergers from recent LIGO/Virgo observations. If, in fact. angular momentum transport in massive stars is efficient, then any (electromagnetic or gravitational wave) observation of a rapidly spinning BH would indicate either a very effective tidal spin up of the progenitor star (homogeneous evolution, high-mass X-ray binary formation through case A mass transfer, or a spin- up of a Wolf-Rayet star in a close binary by a close companion), significant mass accretion by the hole, or a BH formation through the merger of two or more BHs (in a dense stellar cluster).

Key words. stars: massive - black hole physics - gravitational waves

\footnotetext{
^ Our updated models of BH-BH, BH-NS and NS-NS mergers are now publicly available at www. syntheticuniverse.org under the tab "Download/2020: Double Compact Objects/Belczynski et al. 2020"
} 


\section{Introduction}

The LIGO/Virgo Collaboration has reported the detection of ten binary black hole $(\mathrm{BH}-\mathrm{BH})$ mergers during the $\mathrm{O} 1 / \mathrm{O} 2$ observations: GW150914, GW151012, GW151226, GW170104, GW170608, GW170729, GW170809, GW170814, GW170818, and GW170823 (Abbott et al. 2019a,b). We list the basic properties of these mergers in Table 1.

For our analysis, we only used the LIGO/Virgo-reported events; however, three additional BH-BH mergers - GW170121, GW170304, and GW170727 - have been detected with a high level of confidence $\left(P_{\text {astro }}>0.98^{1}\right)$ by Venumadhav et al. (2019). They have low effective spins that are consistent with the triggers reported by the LIGO/Virgo collaboration. The three other events reported by Venumadhav et al. (2019) are not likely to be of astrophysical origin. In particular, GW170403, with a highly negative effective spin, has been reported as the least secure event. Two more events were reported with high effective spins: one with $\chi_{\text {eff }}=0.7_{-0.3}^{+0.2}$ (Venumadhav et al. 2019) and the second with $\chi_{\text {eff }}=0.81_{-0.21}^{+0.15}$ (Zackay et al. 2019); however, these triggers have only a $56 \%$ and $71 \%$ chance of being of astrophysical origin, respectively.

The majority of the reported mergers contain "heavy" BHs with component masses $>20 M_{\odot}$ and they are consistent with being formed by isolated binary evolution of stars with metallicities $\lesssim 10 \% Z_{\odot}$ and initial masses in the range of $40-100 M_{\odot}$, while lower mass BHs may have formed from lower mass stars or at higher metallicity (Belczynski et al. 2010a, 2016a). A typical evolution involves the interaction of stars in a binary through mass transfer and a common envelope phase (Tutukov \& Yungelson 1993; Lipunov et al. 1997; Belczynski et al. 2002; Voss \& Tauris 2003; Dominik et al. 2012; Belczynski et al. 2016b; Eldridge \& Stanway 2016; Woosley 2016; Stevenson et al. 2017; Kruckow et al. 2018; Hainich et al. 2018; Marchant et al. 2019; Spera et al. 2019) and its outcome was predicted to produce the first LIGO/Virgo sources (Belczynski et al. 2010b).

The dynamical formation scenario of $\mathrm{BH}-\mathrm{BH}$ mergers is an evolutionary channel alternative to isolated binary evolution that could operate in globular clusters, nuclear clusters or open, young clusters (Miller \& Hamilton 2002a,b; Portegies Zwart et al. 2004; Gültekin et al. 2004, 2006; O'Leary et al. 2007; Sadowski et al. 2008; Downing et al. 2010; Benacquista \& Downing 2013; Bae et al. 2014; Chatterjee et al. 2017; Mapelli 2016; Hurley et al. 2016; Rodriguez et al. 2016a; VanLandingham et al. 2016; Askar et al. 2017; Arca-Sedda \& Capuzzo-Dolcetta 2019; Samsing 2018; Morawski et al. 2018; Banerjee 2018; Di Carlo et al. 2019; Zevin et al. 2019; Rodriguez et al. 2018; Perna et al. 2019). The dynamical channel can also explain the range of $\mathrm{BH}$ masses observed by LIGO/Virgo.

However, these two basic scenarios, dynamical and isolated, predict different spin-orbit misalignment distributions, with dynamical formation generating nearly isotropic distributions (Mandel \& O'Shaughnessy 2010; Rodriguez et al. 2016b), while binary evolution favors distributions that show mostly small to moderate misalignments, with only a small fraction of mergers reaching high misalignments (Wysocki et al. 2018; Gerosa et al. 2018). A hybrid BH-BH merger formation channel that involves isolated triple (dynamically interacting) star systems (Antonini et al. 2017) favors $\mathrm{BH}$ spin vectors that are located in the $\mathrm{BH}-\mathrm{BH}$ orbital plane, with $\sim 90^{\circ}$ misalignment with respect to the orbital angular momentum if the tertiary dominates the angular momentum of the system (Antonini et al. 2018).

\footnotetext{
1 LIGO/Virgo uses $>0.95$ probability to report events of astrophysical origin.
}

The spin orientations of the BHs can therefore provide important information about their formation (Farr et al. 2017, 2018; Vitale et al. 2017a). However, the effect of spin is subdominant in gravitational waveforms, so spins are more difficult to measure than masses. The waveform is most sensitive to the binary's effective spin,

$\chi_{\mathrm{eff}} \equiv \frac{M_{\mathrm{BH} 1} a_{\mathrm{spin} 1} \cos \Theta_{1}+M_{\mathrm{BH} 2} a_{\mathrm{spin} 2} \cos \Theta_{2}}{M_{\mathrm{BH} 1}+M_{\mathrm{BH} 2}}$,

with $a_{\text {spin } 1,2}$ being the $\mathrm{BH}$ spin magnitudes and $\Theta_{1,2}$ being the angles between the $\mathrm{BH}$ spins and the orbital angular momentum. The dimensionless $\mathrm{BH}$ spin magnitude is defined as:

$a_{\text {spin }} \equiv \frac{c J_{\mathrm{BH}}}{G M_{\mathrm{BH}}^{2}}$,

where $c$ is the speed of light, $G$ is the gravitational constant, and $M_{\mathrm{BH}}$ and $J_{\mathrm{BH}}$ are, respectively, the mass and angular momentum of the BH.

All ten of the BH-BH binaries observed by LIGO/Virgo are consistent with effective spins near zero. Indeed, within the $90 \%$ confidence levels reported by LIGO/Virgo all mergers are consistent with $-0.1 \lesssim \chi_{\text {eff }} \lesssim 0.1$ (see Table 1 ). If the BH spin magnitudes are large, then either (i) both black hole spin vectors are close to the orbital plane, so that $\cos \Theta_{1,2} \approx 0$, or (ii) the black hole angular momenta are nearly equal in magnitude but close to oppositely directed. Alternatively, small values of the effective spin parameter may be obtained if (iii) $\mathrm{BH}$ spin magnitudes are small.

In this study, we investigate the third possibility, namely, the approach that allows the spin magnitudes of $\mathrm{BH}$ in $\mathrm{BH}-\mathrm{BH}$ mergers detected by LIGO/Virgo to be small. Our study is limited to the classical isolated binary evolution $\mathrm{BH}-\mathrm{BH}$ formation scenario. We test several models of natal $\mathrm{BH}$ spins to predict the effective spin parameters of $\mathrm{BH}-\mathrm{BH}$ mergers in the local Universe and to compare them with LIGO/Virgo observations. Compared to Belczynski et al. (2016a,b,c), we incorporated updated mass loss by pair-instability pulsation supernovae, revised our model of accretion onto BHs in close binaries, allowed for effective tidal spin-up of Wolf-Rayet stars, and adopted a new model for the evolution of metallicity and the star formation rate across cosmic time.

Here we briefly summarize the most important ingredients and results of our study. Based on single stellar evolution calculations, we introduce three BH natal spin models (see Sect. 2.1) and we argue that these models can be reasonably used in binary evolution as implemented in our calculations (see Sect. 4.2). We show that initial star rotation does not play a significant role, while angular momentum transport plays crucial role in setting the BH natal spin (see Sect. 4.1). In binary evolution, accretion and mass transfer (see Sect. 2.4) do not play significant role, while tidal interactions (see Sect. 2.5) may increase $\mathrm{BH}$ spins for about $20-30 \%$ of the BH-BH mergers (see Sect. 3.4). Merger rates for $\mathrm{BH}-\mathrm{BH}$, neutron star-neutron star (NS-NS), and black hole-neutron star (BH-NS) binaries are sensitive to assumptions regarding the common envelope and natal kicks, but they also strongly depend on the assumed cosmic chemical evolution model, while the change of merger rates with redshift depends mostly on the cosmic star-formation history (see Sect. 3.2).

\section{Model}

\subsection{Natal black hole spin}

Various rotating star models differ in terms of the physics of their rotation and, in particular, the efficiency of angular 
Table 1. LIGO/Virgo O1/O2 BH-BH mergers.

\begin{tabular}{lccccc}
\hline \hline Event & $\chi_{\text {eff }}$ & $M_{\text {tot }}$ & $M_{1}$ & $M_{2}$ & $z$ \\
\hline GW150914 & -0.01 & 62.8 & 35.6 & 30.6 & 0.09 \\
& {$[-0.14,0.11]$} & {$[59.9,65.9]$} & {$[32.6,40.4]$} & {$[26.2,33.6]$} & {$[0.06,0.12]$} \\
GW151012 & 0.04 & 36.3 & 23.3 & 13.6 & 0.21 \\
& {$[-0.15,0.32]$} & {$[33.1,45.2]$} & {$[17.8,37.3]$} & {$[8.8,17.7]$} & {$[0.12,0.30]$} \\
GW151226 & 0.18 & 20.6 & 13.7 & 7.7 & 0.09 \\
& {$[0.06,0.38]$} & {$[19.3,25.7]$} & {$[10.5,22.5]$} & {$[5.1,9.9]$} & {$[0.05,0.13]$} \\
GW170104 & -0.04 & 50.4 & 31.0 & 20.1 & 0.19 \\
& {$[-0.24,0.13]$} & {$[46.7,55.0]$} & {$[25.4,38.2]$} & {$[15.6,25.0]$} & {$[0.11,0.26]$} \\
GW170608 & 0.03 & 17.7 & 10.9 & 7.6 & 0.07 \\
& {$[-0.04,0.22]$} & {$[17.1,20.7]$} & {$[9.2,16.2]$} & {$[5.5,8.9]$} & {$[0.05,0.09]$} \\
GW170729 & 0.36 & 86.3 & 50.6 & 34.3 & 0.48 \\
& {$[0.11,0.57]$} & {$[75.3,100]$} & {$[40.4,67.2]$} & {$[24.2,43.4]$} & {$[0.28,0.67]$} \\
GW170809 & 0.07 & 57.9 & 35.2 & 23.8 & 0.20 \\
& {$[-0.09,0.23]$} & {$[54.3,63.2]$} & {$[29.2,43.5]$} & {$[18.7,29.0]$} & {$[0.13,0.25]$} \\
GW170814 & 0.07 & 54.1 & 30.7 & 25.3 & 0.12 \\
& {$[-0.04,0.19]$} & {$[51.7,57.3]$} & {$[27.7,36.4]$} & {$[21.2,28.2]$} & {$[0.08,0.15]$} \\
GW170818 & -0.09 & 61.3 & 35.5 & 26.8 & 0.20 \\
& {$[-0.29,0.09]$} & {$[57.7,65.8]$} & {$[30.8,43.0]$} & {$[21.6,31.1]$} & $\mathrm{v}[0.13,0.27]$ \\
GW170823 & 0.08 & 69.1 & 39.6 & 29.4 & 0.34 \\
& {$[-0.14,0.28]$} & {$[62.3,78.4]$} & {$[33.0,49.6]$} & {$[22.3,28.6]$} & {$[0.20,0.47]$} \\
\hline
\end{tabular}

Notes. For all parameters, average and $90 \%$ confidence limits are listed, $\chi_{\text {eff }}$ : effective spin parameter, $M_{\mathrm{tot}}$ : total intrinsic BH-BH binary mass, $M_{1}$ : primary $\mathrm{BH}$ intrinsic mass, $M_{2}$ : secondary $\mathrm{BH}$ intrinsic mass, $z$ : event redshift.

momentum transport. Here we test three different models of angular momentum transport for massive stars. We adopted moderate angular momentum transport, along with Geneva stellar models (Eggenberger et al. 2008; Ekström et al. 2012) that are based on Zahn (1992) theory, in which angular momentum is mainly transported by meridional currents: the shellular model. Efficient angular momentum transport was adopted with standard MESA stellar models (calculated in this study), which use an efficient transport mechanism mediated by the action of the so-called Tayler-Spruit magnetic dynamo in the radiative zone (Spruit 1999, 2002). Super-efficient angular momentum transport is based on the Tayler-Spruit dynamo, which is subsequently modified to include stronger magnetic field generation that allows for more efficient angular momentum transport (Fuller et al. 2019; Ma \& Fuller 2019; Fuller \& Ma 2019).

To test the validity of each of these models through gravitational wave astrophysics, we compared their predictions with the LIGO/Virgo effective-spin estimates. For the Geneva (moderate) and MESA (efficient) angular momentum transport, we use models based on a wide range of metallicity: $Z=0.014$; 0.006; 0.002; 0.0004 (see Appendices A.1 and A.2). We assume that stars on the zero age main sequence (ZAMS) have an equatorial velocity equal to $40 \%$ of the critical velocity, defined as the velocity at which the centrifugal acceleration completely balances gravity ${ }^{2}$. To test the importance of the initial rotation rates, we ran several models based on different assumptions about this initial condition; for more, see Sect. 4.1 and Figs. 23 and A.1. We find that the final core rotation rate is almost inde-

\footnotetext{
2 GENEC and MESA use two slightly different definitions of the critical velocity. Maeder \& Meynet (2000) discusses the two definitions. MESA models use their Eq. (3.12), whereas GENEC models use the minimum between the values obtained from their Eqs. (3.14) and (3.19), respectively.
}

pendent of the initial rotation rate but depends strongly on the angular momentum transport process included in the simulation (i.e., non-magnetic or magnetic). For the Fuller (superefficient) model of angular momentum transport, we assume that the natal BH spin depends neither on initial stellar rotation nor on metallicity, since in each case almost all angular momentum is removed from the stellar core (Fuller et al. 2019; Ma \& Fuller 2019; Fuller \& Ma 2019).

The stellar models provide the angular momentum in each zone that corresponds to a spherical shell in the star. We assume that angular momentum is conserved in the collapse phase and we calculate the angular momentum of the compact remnant by summing the angular momentum of the zones with enclosed mass lower than the compact remnant mass.

From the amount of angular momentum contained in the collapsing core, we calculate the dimensionless BH spin magnitude $a_{\text {spin }}$. However, we limit the spin magnitude to 0.9: $a_{\text {spin }}=$ $\min \left(a_{\text {spin }}, 0.9\right)$ to account for any potential processes that might remove some angular momentum during $\mathrm{BH}$ formation. We arbitrarily picked 0.9 as a maximum for the spin rate. The difficulty in predicting the fastest spin values is that they require highangular momentum, disk-forming infalls. There are several spurious mechanisms which can remove angular momentum from these accretion disks, but for now we do not have means to perform predictive calculations of this effect. Disk winds are one example, yet the actual amount of material and angular momentum that is removed by such winds can vary significantly (Vlahakis \& Königl 2001; Surman et al. 2006; Janiuk 2017). Therefore, we have used $a_{\text {spin }}=0.9$ to denote any value between 0.9 and 1.0, depending on these accretion loss mechanisms.

In Figs. 1 and 2, we show the BH spins as a function of the progenitor's CO core mass for the Geneva and MESA models. We approximate the natal BH spin by simple fits. These rough 


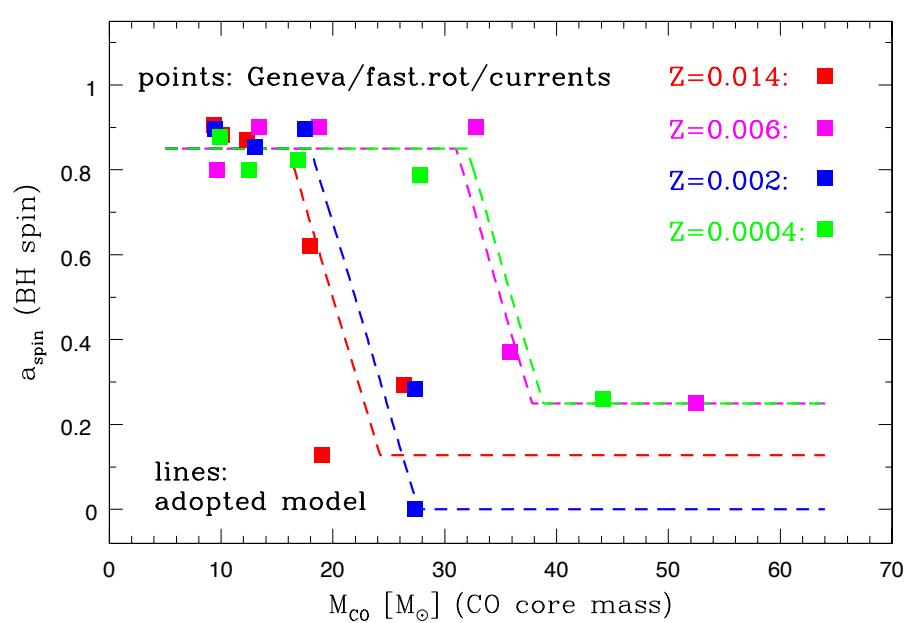

Fig. 1. Magnitude of natal $\mathrm{BH}$ spin as a function of the $\mathrm{CO}$ core mass of the collapsing star for the Geneva stellar models with $40 \%$ critical initial velocity and mild angular momentum transport by meridional currents. Lines mark our adopted model for natal BH spins and its dependence on metallicity (see Eq. (3)). The star's CO core mass may be used as a proxy for the $\mathrm{BH}$ mass (see Appendix A.3).

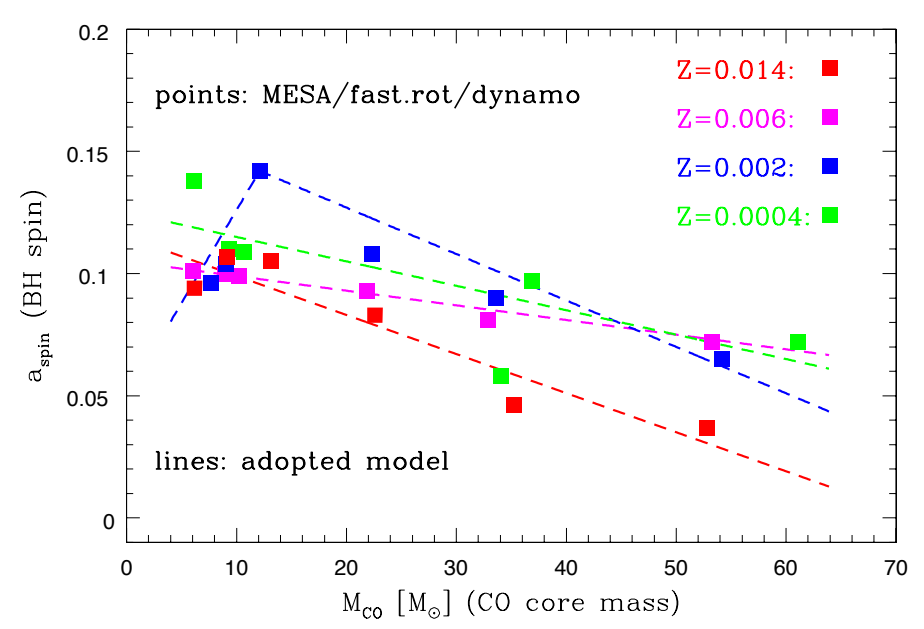

Fig. 2. Magnitude of natal $\mathrm{BH}$ spin as a function of the $\mathrm{CO}$ core mass of the collapsing star for the MESA stellar models with $40 \%$ critical initial velocity and the Tayler-Spruit magnetic dynamo (efficient) angular momentum transport. Lines mark our adopted model for natal BH spins and its dependence on metallicity (see Eq. (4)).

fits are meant to reproduce the general trends in the data and are used in our population synthesis calculations. We list the actual data in Tables A.1 and A.2, so other fits can be attempted if desired.

For the Geneva models the natal BH spin may be approximated by:

$a_{\text {spin }}=\left\{\begin{array}{ll}0.85 & M_{\mathrm{CO}} \leq m_{1} \\ a M_{\mathrm{CO}}+b & m_{1}<M_{\mathrm{CO}}<m_{2} \\ a_{\text {low }} & M_{\mathrm{CO}} \geq m_{2}\end{array}\right.$,

with $a=-0.088$ for all models; $b=2.258, m_{1}=16.0 M_{\odot}$, $m_{2}=24.2 M_{\odot}, a_{\text {low }}=0.13$ for $Z=0.014 ; b=3.578, m_{1}=$ $31.0 M_{\odot}, m_{2}=37.8 M_{\odot}, a_{\text {low }}=0.25$ for $Z=0.006 ; b=2.434$, $m_{1}=18.0 M_{\odot}, m_{2}=27.7 M_{\odot}, a_{\text {low }}=0.0$ for $Z=0.002$; and $b=3.666, m_{1}=32.0 M_{\odot}, m_{2}=38.8 M_{\odot}, a_{\text {low }}=0.25$ for $Z=0.0004$. We note that progenitor stars with CO cores less massive than $\sim 20 M_{\odot}$ (low- to intermediate-mass BHs) tend to produce high-spin BHs $\left(a_{\text {spin }} \sim 0.8-0.9\right)$, but higher mass stars (massive BHs) tend to produce low-spin BHs $\left(a_{\text {spin }} \sim 0-0.3\right)$. This general trend is easily understood. Stellar winds during the evolution of a massive star can carry away a considerable amount of angular momentum (Meynet et al. 2015). For the most massive stars, this mass loss is extensive, effectively removing angular momentum and producing low-spin BHs. The data points also show a non-monotonic dependence on metallicity, which is the result of a complex and metallicity-dependent interplay between the strength of stellar winds, the extent of the H-burning shell, and the model for the efficiency of element diffusion within the meridional current (see Appendix A.1). by:

For MESA models the natal $\mathrm{BH}$ spin may be approximated

$a_{\text {spin }}=\left\{\begin{array}{ll}a_{1} M_{\mathrm{CO}}+b_{1} & M_{\mathrm{CO}} \leq m_{1} \\ a_{2} M_{\mathrm{CO}}+b_{2} & M_{\mathrm{CO}}>m_{1}\end{array}\right.$,

with $a_{1}=-0.0016, b_{1}=0.115, m_{1}=\infty$ for $Z=0.014 ; a_{1}=$ $-0.0006, b_{1}=0.105, m_{1}=\infty$ for $Z=0.006 ; a_{1}=0.0076$, $b_{1}=0.050, a_{2}=-0.0019, b_{2}=0.165, m_{1}=12.09 M_{\odot}$ for $Z=0.002$; and $a_{1}=-0.0010, b_{1}=0.125, m_{1}=\infty$ for $Z=$ 0.0004 . The MESA models include the Tayler-Spruit magnetic dynamo and thus models of all masses and at all metallicities end up with low $\mathrm{BH}$ spin magnitudes in the range of $0.05 \lesssim a \lesssim 0.15$. There is a mild tendency for lower metallicity and lower initial mass models to end up with slightly higher BH spin magnitudes but the dependence is much weaker than in the Geneva models, which do not include magnetic field related transport of angular momentum.

Finally, for the Fuller model that employs super-efficient angular momentum transport, we adopt a single value of $\mathrm{BH}$ natal spin for stars of all masses and all metallicities:

$a_{\text {spin }}=0.01$.

We note that single stellar models (of different mass and metallicity) presented by Fuller \& Ma (2019) produce very low spins for BHs in the range of $a=0.003-0.035$, with a typical value of $a \sim 0.01$.

In our binary population synthesis calculations, we employ the above-presented natal BH spin estimates obtained from single stellar evolution (see Sect. 2.7). The discussion of binary interactions that can affect these natal $\mathrm{BH}$ spins (that are and are not taken into account) is given in Sect. 4.2.

\subsection{Black hole spin misalignment}

To calculate the effective spin of $\mathrm{BH}-\mathrm{BH}$ mergers we need to know the misalignment angles of the two $\mathrm{BH}$ spin vectors with respect to the orbital angular momentum vector: $\Theta_{1}$ and $\Theta_{2}$ (see Eq. (1)).

In our estimation of these angles, we ignore the potential effects of tides and mass transfer and accretion; see Gerosa et al. (2013) and Wysocki et al. (2018) for alternatives and further discussion. However, in our calculations, we take into account the binary components spin precession. We assume that the two stars are born with spins that are fully aligned with the ZAMS binary orbital angular momentum $\left(\boldsymbol{L}_{0}\right)$. At each $\mathrm{BH}$ formation, the natal kick may change the orbit and its orientation in space. After the first $\mathrm{BH}$ formation, the new orbital angular momentum is $\boldsymbol{L}_{1}$, whereas following the second $\mathrm{BH}$ formation it is $\boldsymbol{L}_{2}$. After the 
first $\mathrm{BH}$ formation, if there is a natal kick, the binary component spins $\boldsymbol{S}_{1}$ and $\boldsymbol{S}_{2}$ are not aligned with the binary angular momentum vector. We assume that after the first $\mathrm{BH}$ formation both the $\mathrm{BH}$ spin and the companion star spin are small compared to the binary orbital angular momentum. With this assumption, the total angular momentum of the binary is equal to the orbital angular momentum. Therefore, both the binary component spins precess around $\boldsymbol{L}_{1}$. The precession periods for the $\mathrm{BH}$ and the companion star spin are different. In this approximation the spin of the BH (or the star) can be described as a sum of two components $S_{1 / 2}=S_{1 / 2}^{\|}+S_{1 / 2}^{\perp}$, where $S_{1 / 2}^{\|}$is the spin component parallel to the orbital angular momentum and $S_{1 / 2}^{\perp}$ is the spin component perpendicular to the orbital angular momentum. During precession the former is constant and the latter has a constant value but rotates in the plane perpendicular to the direction of the angular orbital momentum. The precession period of each spin is different (Hamilton \& Sarazin 1982). Thus, at the time of the second $\mathrm{BH}$ formation we chose random positions of each component vector on its precessing trajectory and they become $S_{1}^{\prime}$ and $S_{2}^{\prime}$. The natal kick (if any) changes again the binary orbit orientation in space, which then becomes $\boldsymbol{L}_{2}$. We followed all these changes to calculate the effective spin:

$\chi_{\mathrm{eff}}=\frac{M_{\mathrm{BH} 1} \boldsymbol{S}_{1}^{\prime}+M_{\mathrm{BH} 2} \boldsymbol{S}_{2}^{\prime}}{M_{\mathrm{BH} 1}+M_{\mathrm{BH} 2}} \cdot \frac{\boldsymbol{L}_{2}}{\left|\boldsymbol{L}_{2}\right|}$.

We note that although (after the second $\mathrm{BH}$ formation) misaligned BH spins are subject to precession, the value of $\chi_{\text {eff }}$ is expected to remain constant during the long inspiral towards the LIGO/Virgo band (Gerosa et al. 2015).

\subsection{Black hole masses}

In our calculation of BH masses, we use formulas assuming both the rapid and delayed supernova engines (Fryer et al. 2012). The rapid development of the engine naturally creates a mass gap between NSs and BHs (dearth of compact objects in the range of $2-5 M_{\odot}$ ), while the delayed engine does produce compact objects in this range (Belczynski et al. 2012a). For singlestar evolution, the delayed model minimum BH mass is $2.5 M_{\odot}$, while it is $5 M_{\odot}$ for the rapid model. We note that binary evolution may create light BHs $\left(\sim 2.5-5 M_{\odot}\right)$ by accretion induced collapse of a NS to a $\mathrm{BH}$ independent of supernova model (Belczynski \& Taam 2004).

The maximum mass of a $\mathrm{BH}$ is set by the maximum mass of a star and wind mass loss rates and is sensitive to the potential explosive mass-loss during the final stages of the star's life. We adopted a rather conservative maximum initial mass for the stars: $M_{\text {ZAMS }}<150 M_{\odot}$, although there appears to be evidence that stars with masses of $\sim 200-300 M_{\odot}$ may exist even in the local Universe (Crowther et al. 2010). For stellar winds we adopt formulas based on theoretical predictions of radiation driven mass loss (Vink et al. 2001) with inclusion of Luminous Blue Variable mass loss (Belczynski et al. 2010a). These wind mass loss rates may be overestimated by as much as one order of magnitude (Oskinova et al. 2011; Ramachandran et al. 2019). Therefore, we allow (conservatively) for the reduction of stellar wind mass loss rates to $f_{\text {wind }}=0.3$ of their currently adopted values.

We also allow for pair-instability supernovas (PSNs) to entirely disrupt massive stars (stars with He core mass in the range of $M_{\mathrm{He}}=65-135 M_{\odot}$ ) and leaving no NS/BH remnant. For somewhat lower-mass stars (stars with He core mass in the range of $M_{\mathrm{He}} \approx 40-65 M_{\odot}$ ), we allow for pair-instability pulsation supernovas (PPSNs). This process may remove the outer

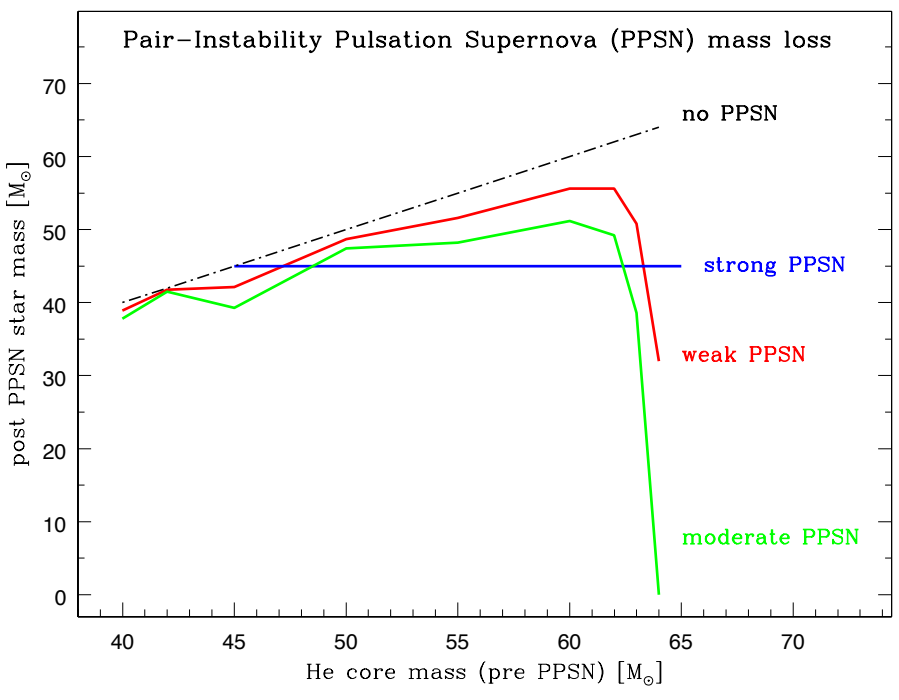

Fig. 3. Adopted models for pair-instability pulsation supernova mass loss. For a given He core mass we show the mass of a star after PPSN mass loss. Moderate PPSN mass loss is adopted directly from Leung et al. (2019), while its modified (50\% reduced mass loss) version is presented as weak PPSN model. Strong PPSN are adopted from Belczynski et al. (2016c).

layers of a star, but does not lead to its disruption and allows for the BH formation in core collapse. We adopt three models for PPSN mass loss. In the first model, we adopt strong PPSNs that are assumed to always remove the entire star mass above the inner $45.0 M_{\odot}$ (Belczynski et al. 2016c) for stars with $M_{\mathrm{He}}=45-65 M_{\odot}$. Therefore, the post PPSN star mass is:

$M_{\text {star }} / M_{\odot}=45.0 \quad 45.0 \leq M_{\mathrm{He}}<65.0 M_{\odot}$.

In the second model, we adopt recent PPSN calculations that allow for as much as $51.2 M_{\odot}$ of the star to remain bound after a PPSN (Leung et al. 2019). In this moderate PPSN model, the explosive mass loss depends on the He core mass. The post PPSN star mass is:

$$
M_{\text {star }} / M_{\odot}= \begin{cases}0.65 M_{\mathrm{He}}+12.2 & 40.0 \leq M_{\mathrm{He}}<60.0 M_{\odot} \\ 51.2 & 60.0 \leq M_{\mathrm{He}}<62.5 M_{\odot} \\ -14.3 M_{\mathrm{He}}+938.0 & 62.5 \leq M_{\mathrm{He}} \leq 65.0 M_{\odot} .\end{cases}
$$

Finally, we adopt a weak PPSN model that allows only for $50 \%$ of the mass loss calculated by Leung et al. (2019). In this scheme, the post PPSN star mass may reach 55.6 $M_{\odot}$, and we approximate the post PPSN star mass with:

$$
M_{\mathrm{star}} / M_{\odot}= \begin{cases}0.83 M_{\mathrm{He}}+6.0 & 40.0 \leq M_{\mathrm{He}}<60.0 M_{\odot} \\ 55.6 & 60.0 \leq M_{\mathrm{He}}<62.5 M_{\odot} \\ -14.3 M_{\mathrm{He}}+938.1 & 62.5 \leq M_{\mathrm{He}} \leq 65.0 M_{\odot} .\end{cases}
$$

All three models are shown in Fig. 3. For the two models based on calculations from Leung et al. (2019), we note a steep decrease in post PPSN star mass for He core masses above $\sim 60 M_{\odot}$. The masses of these He stars are very close to the boundary mass between the PPSN and the PSN. As the mass of the He star increases, the central temperature at the bounce increases and oxygen burning becomes more explosive and causes a larger amount of mass ejection. Eventually, explosive oxygen burning in the $\sim 65 M_{\odot}$ He star produces large 
enough nuclear energy to disrupt the star completely with no BH remnant, in other words, it induces PSN. The amount of mass ejection increases steeply as the He star mass increases from $\sim 60 M_{\odot}$ to $\sim 65 M_{\odot}$ because the oxygen burning rate is very sensitive to the temperature. Thus the remnant BH mass decreases steeply as the He star mass approaches $65 M_{\odot}$.

In the PPSN mass regime, massive BHs are formed and, according to our scheme (Fryer et al. 2012), these BHs form through collapse of the entire star into a $\mathrm{BH}$ (direct $\mathrm{BH}$ formation). However, we allow for some mass loss in neutrinos. In the original formulas we have adopted a high neutrino fractional mass loss ( $f_{\text {neu }}=0.1$; see e.g., Belczynski et al. 2016a), whereas, here, we also allow also for much smaller mass loss in some models $\left(f_{\text {neu }}=0.01\right)$. The final BH mass, in case of the direct $\mathrm{BH}$ formation, is calculated from:

$M_{\mathrm{BH}}=\left(1-f_{\text {neu }}\right) M_{\mathrm{star}}$,

where $M_{\text {star }}$ denotes the star's mass just prior the core collapse.

\subsection{Black hole accretion model}

Mass accretion may increase the $\mathrm{BH}$ mass and spin after its formation. Here we test two models of accretion from a stable Roche lobe overflow (RLOF; not common envelope [CE]) mass-transfer or from stellar winds. The first model (efficient $\mathrm{BH}$ accretion) is based on the results of global, axisymmetric simulations of accretion disks with $\alpha P$ viscosity, disk winds and photon trapping performed by Ohsuga (2007). Belczynski et al. (2008a) obtained a fit to the results of Ohsuga (2007):

$$
\log \left(\frac{\dot{M}_{\mathrm{BH}}}{\dot{M}_{\text {crit1 }}}\right)= \begin{cases}\log \left(\frac{\left|\dot{M}_{\text {don }}\right|}{\dot{M}_{\text {crit }}}\right) & \left|\dot{M}_{\text {don }}\right| \leq \dot{M}_{\text {crit1 }} \\ 0.544 \log \left(\frac{\left|\dot{M}_{\text {don }}\right|}{\dot{M}_{\text {crit }}}\right) & \dot{M}_{\text {crit1 } 1}<\left|\dot{M}_{\text {don }}\right| \leq 10 \times \dot{M}_{\text {crit1 }} \\ 0.934 \log \left(\frac{\left|\dot{M}_{\text {don }}\right|}{\dot{M}_{\text {critl }}}\right)-0.380 & \left|\dot{M}_{\text {don }}\right|>10 \times \dot{M}_{\text {crit1 }}\end{cases}
$$

where $\dot{M}_{\mathrm{BH}}$ is the mass accumulation rate onto the BH, $\dot{M}_{\text {crit1 }}=$ $2.6 \times 10^{-8}\left(M_{\mathrm{BH}} / 10 M_{\odot}\right) M_{\odot} \mathrm{yr}^{-1}$ is the critical mass transfer rate (obtained from numerical simulations) above which the accretion onto the BH is not fully efficient, and $\dot{M}_{\text {don }}$ is the mass transfer rate from the donor to the accretion disk around the $\mathrm{BH}$. We note that above the critical mass transfer rate some mass transferred from the donor is ejected from the system.

The second model (inefficient BH accretion) uses the analytical prescription of Shakura \& Sunyaev (1973). In this model, disk winds play a significant role effectively limiting the mass accumulation for super-Eddington mass transfer rates by limiting the local accretion rate to its Eddington value. This model is supported by the numerical results of strong outflow from the super-critical accretion disk (Abolmasov et al. 2009; Sadowski et al. 2016), as well as by observations of many ultra-luminous $\mathrm{X}$-ray sources in centers of unusually large and bright emission nebulae which are powered by the accumulated kinetic energy of the outflow (Pakull \& Mirioni 2003; King 2004), and also supported by the fact that the neutron star in Cygnus X-2 while being subject to high mass transfer rate $\left(\sim 10^{-5} M_{\odot} \mathrm{yr}^{-1}\right)$ has ejected most of the mass transferred from the donor star (King \& Ritter 1999).

In this model we assume that the mass accumulation rate onto the $\mathrm{BH}$ is given by:

$\dot{M}_{\mathrm{BH}}=f_{1} f_{2} \dot{M}_{\mathrm{don}}$,

where $\left(1-f_{1}\right)$ is the fraction of the mass transferred to the disk from the donor which is lost in wind from the outer part of the accretion disk. It is difficult to determine the wind mass loss rate from the outer part of the disk (see Sadowski et al. 2016), and we treat $f_{1}$ as a parameter in our model. For the current calculations we adopt $f_{1}=1$, indicating that there is no wind mass loss in the outer part of the disk, which maximizes accumulation of mass onto the BH. Similarly, $\left(1-f_{2}\right)$ represents the fraction of mass which is lost from the inner part of the accretion disk. The value of $f_{2}$ depends on the mass transfer rate as:

$f_{2}=\left\{\begin{array}{ll}1, & \text { for } \dot{M}_{\text {don }} \leq \dot{M}_{\text {crit2 }} \\ \frac{R_{\mathrm{ISCO}}}{R_{\text {sph }}}, & \text { for } \dot{M}_{\text {don }}>\dot{M}_{\text {crit2 }}\end{array}\right.$,

where the critical mass transfer rate is $\dot{M}_{\text {crit2 }}=\dot{M}_{\text {Edd }}=4.375 \times$ $10^{-8}(1+X)^{-1}\left(M_{\mathrm{BH}} / M_{\odot}\right) M_{\odot} \mathrm{yr}^{-1}$, where the hydrogen mass fraction $X$ is 0.7 for $\mathrm{H}$-rich donor stars and 0.0 for $\mathrm{H}$-deficient donor stars, $R_{\mathrm{ISCO}}$ is the innermost stable circular orbit radius, and $R_{\text {sph }}$ is the spherisation radius, where the disk's height becomes comparable to the radius. The $R_{\mathrm{ISCO}}$ depends on the spin value of the accreting $\mathrm{BH}$ and varies between $0.5 R_{\mathrm{S}}$ (for a maximally prograde spinning $\mathrm{BH}$ ) to $4.5 R_{\mathrm{S}}$ (for a maximally retrograde spinning $\mathrm{BH}$ ), where $R_{\mathrm{S}}=\frac{2 G M_{\mathrm{BH}}}{c^{2}}$ is the Schwarzchild radius of a BH. The $R_{\text {sph }}$ is given by (Shakura \& Sunyaev 1973) as:

$R_{\mathrm{sph}}=\frac{27}{4} \frac{\dot{M}_{\mathrm{don}}}{\dot{M}_{\mathrm{Edd}}} R_{\mathrm{S}}$

The two equations above make up a simplified description of the change of the accretion mode onto compact objects. In particular, they lead to a jump in mass accretion rate onto a compact object when mass transfer from a donor star is equal to the critical mass transfer rate $\left(M_{\text {don }}=\dot{M}_{\text {crit } 2}\right)$. This simplification does not influence the results of our evolutionary calculations.

In our calculations, we always assume a prograde $\mathrm{BH}$ spin, and the initial $\mathrm{BH}$ spin magnitude is adopted from a given stellar model (see Sect. 2.1) and then increased by accretion as detailed in Belczynski et al. (2008a). At sub-Eddington accretion rates $\left(\dot{M}_{\text {don }} \leq \dot{M}_{\text {Edd }}\right)$, we assume that there is no mass loss from the inner part of the disk $\left(f_{2}=1\right)$.

The mass accumulation onto a $\mathrm{BH}$ in the inefficient $\mathrm{BH}$ accretion model is always Eddington-limited $\left(\dot{M}_{\mathrm{BH}}<\dot{M}_{\mathrm{Edd}}\right)$, whereas in the efficient $\mathrm{BH}$ accretion model it increases monotonically with the mass transfer rate and may significantly exceed $\dot{M}_{\text {Edd }}$. These two models produce the same accumulation on a BH for mass transfer rates below $\dot{M}_{\text {crit } 1}=0.06 \times \dot{M}_{\text {Edd }}$ where both models give exactly the same prescription $\left(\dot{M}_{\mathrm{BH}}=\left|\dot{M}_{\mathrm{don}}\right|\right.$, noting that $\dot{M}_{\text {crit1 }}=0.06 \dot{M}_{\text {crit } 2}$ ).

As a result, the evolution of BH binaries which go through a phase of stable mass transfer with high (super-critical) mass transfer rate may be considerably different under different assumptions about the $\mathrm{BH}$ accumulation efficiency. However, the dominant formation channel for BH-BH mergers (also Figs. 8 and 9 in Belczynski et al. 2016a) contains no such phase. Along the way to formation for most $\mathrm{BH}-\mathrm{BH}$ mergers in our models, the accretion of matter by a $\mathrm{BH}$ takes place only during a short-lived $\mathrm{CE}$ event or as result of capturing a fraction of a stellar wind from the companion. Accretion during CE can be best related to the Bondi-Hoyle accretion and has been found to be rather insignificant (less than $\sim 1 M_{\odot}$ for a typical $30 M_{\odot} \mathrm{BH}$, see Appendix A.7 for a detailed discussion of recent calculations of accretion during $\mathrm{CE}$ ). The wind-fed accretion during the subsequent phase of a compact $\mathrm{BH}$ - Wolf-Rayet (BH-WR) binary evolution is even less significant, partially due to small wind-mass loss rates from low metallicity systems (which are the progenitors of most $\mathrm{BH}-$ BH mergers). 


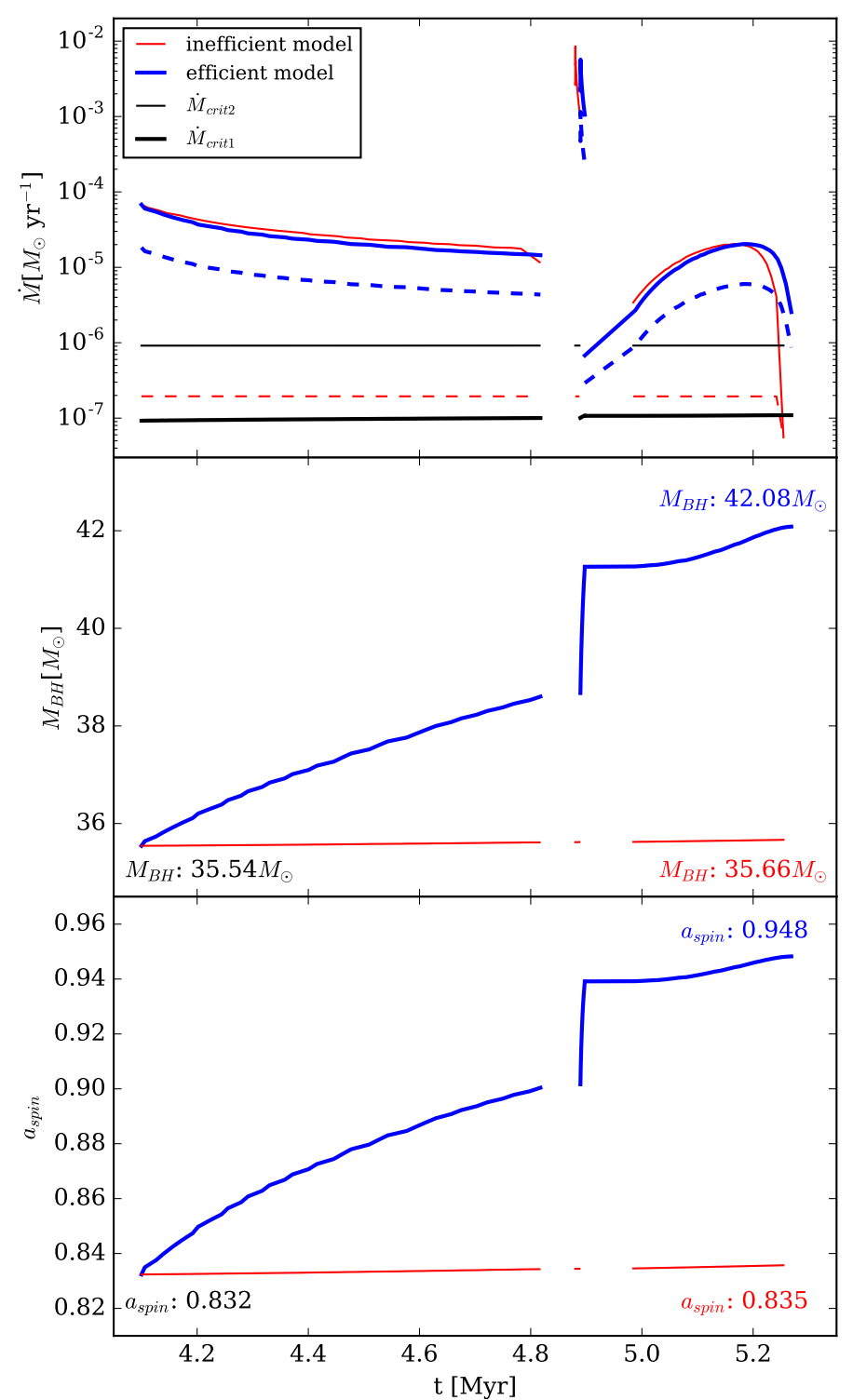

Fig. 4. Comparison of the two black hole accretion models employed in the StarTrack code. We present the evolution of the mass transfer rate from a donor star ( $\dot{M}_{\text {don }}$; top panel, solid lines), BH mass accumulation rate $\left(\dot{M}_{\mathrm{BH}}\right.$; top panel, dashed lines), $\mathrm{BH}$ mass $\left(M_{\mathrm{BH}}\right.$; middle panel), and BH spin $\left(a_{\text {spin }}\right.$; bottom panel) during the RLOF stable mass transfer phases. Critical mass transfer rates (above which mass ejection from a system is expected) are provided for reference. During the first part of the RLOF ( $t \approx 4-4.8 \mathrm{Myr}$ ) the donor is a MS star, during the short-duration peak it is a HG star $(t \approx 4.9 \mathrm{Myr})$, and for the remaining RLOF it is a helium-core burning star. See Sect. 2.4 for a description of the full evolutionary path and both accretion models.

That said, the $\mathrm{BH}$ accretion models presented in this section can play an important role in certain sub-dominant channels for the BH-BH merger formation. In Fig. 4 we showcase the time evolution of the mass transfer and accretion rates as well as the $\mathrm{BH}$ spin and mass in a system in which the $\mathrm{BH}$ accretion is particularly significant. The system began its evolution as two ZAMS stars with masses of $84.6 M_{\odot}$ and $48 M_{\odot}$ formed at a very low metallicity $Z=0.0002$ in a binary with separation of about $1900 R_{\odot}$. At the age of $3.8 \mathrm{Myr}$ the primary, now a $66 M_{\odot}$ helium-core burning star with a radius of $800 R_{\odot}$, goes into RLOF and initiates a CE phase. As a result, the primary becomes a $36 M_{\odot}$ WR star and the binary separation decreases down to $30 R_{\odot}$. With the orbit already being quite compact, the companion MS star $\left(\sim 47 M_{\odot}\right)$ initiates another RLOF and starts stable mass transfer back onto the WR primary. The WR star grows to about $39 M_{\odot}$, before collapsing directly into a $35.54 M_{\odot}$ $\mathrm{BH}$ at the age of $4 \mathrm{Myr}$ (with no natal kick, $10 \%$ of mass being lost in neutrinos, and the natal spin of $a_{\text {init }}=0.832$ adopted from Geneva BH natal spin model). Soon thereafter, the companion MS initiates a RLOF again and the first phase of stable, super-critical mass transfer onto the newly-formed $\mathrm{BH}$ begins $\left(\dot{M}_{\text {don }} \sim\right.$ a few $\left.\times 10^{-5} M_{\odot} \mathrm{yr}^{-1}\right)$. At that moment the system may be potentially observable as an ultraluminous X-ray source (see e.g., Wiktorowicz et al. 2019, for the recent analysis of these objects). The mass transfer continues up until about $t=4.8 \mathrm{Myr}$ (Fig. 4), at which point the donor is at the very end of its MS evolution and contracts a bit, causing a temporary detachment. The mass transfer starts again when the companion begins to expand on its $\mathrm{HG}$ at the age of about $t=5.9 \mathrm{Myr}$ having mass of $28 M_{\odot}$. The rate is high $\left(\sim 10^{-3} M_{\odot} \mathrm{yr}^{-1}\right)$, but the phase is shortlived (7.7 Myr). Finally, the last phase of mass transfer occurs when the companion is a slowly expanding core helium burning star and terminates at about 5.25 Myr.

The net result of the subsequent stable mass transfer phases shown in Fig. 4 is an increase of the binary separation which, at the point of the final detachment, is about $160 R_{\odot}$ (similar in both accretion models). At that point the donor star has been stripped almost down to its helium core and has a remaining mass of only about $10 M_{\odot}$.

Since accumulation of mass onto the $\mathrm{BH}$ (dashed lines in the top panel of Fig. 4) is different in the two models for supercritical mass transfer rates, the final $\mathrm{BH}$ mass is also different: $42.08 M_{\odot}$ for the efficient $\mathrm{BH}$ accretion model and $35.66 M_{\odot}$ for the inefficient $\mathrm{BH}$ accretion model (Fig. 4, middle panel). We also note that the BH spin, was increased to 0.948 for the efficient model and to 0.835 for the inefficient model.

This particular binary ends its evolution at $t \approx 5.6 \mathrm{Myr}$ forming a BH-BH with masses of $35.7 M_{\odot}$ and $6.2 M_{\odot}$ for the inefficient model, or $42.1 M_{\odot}$ and $6.0 M_{\odot}$ for the efficient model. The delay time to merger is about $3 \mathrm{Myr}$. The second $\mathrm{BH}$ is formed through a supernova explosion with a natal kick. As the separation before the supernova explosion was relatively large in both accretion models $\left(\sim 160 R_{\odot}\right)$, the formation of a binary that could merge within the Hubble time was only possible thanks to the preferentially oriented natal kick, which decreased the separation down to $\sim 90 R_{\odot}$ and, more importantly, induced a high eccentricity of $e>0.988$ (from a $e=0.0$ pre-supernova value). Such an influence of a natal kick on the binary orbit is very rare (Andrews \& Zezas 2019), so the above example is an extreme example of a $\mathrm{BH}-\mathrm{BH}$ merger formation. In the vast majority of systems, the impact of the accretion model on the $\mathrm{BH}-\mathrm{BH}$ formation is much smaller.

\subsection{Tidal interactions}

In this section, we discuss the efficiency of tidal spin-up in close binaries. Our standard implementation of tides follows from Hurley et al. (2002) and is based on the standard Zahn (1977, 1989) theory as updated by more recent calibrations (Claret 2007). These prescriptions, which are laid out in Belczynski et al. (2008b), result in rather weak tidal interactions between stars in binary systems. This is particularly true for very massive stars (e.g., progenitors of BHs) that evolve so fast that the tides do not affect significantly their rotation.

In our classical BH-BH formation scenario (Belczynski et al. 2016a), the only evolutionary phase at which tides could spin-up 
either of the $\mathrm{BH}$ progenitors happens after the $\mathrm{CE}$ phase when the initially wide binary $\left(a \gtrsim 1000 R_{\odot}\right)$ is reduced to a rather tight orbit $\left(a \lesssim 100 R_{\odot}\right)$. Such a configuration will consist of at least one stripped stellar core (WR star): BH-WR, WR-BH or WR-WR binaries.

Recently Kushnir et al. (2016, 2017), Zaldarriaga et al. (2018), Hotokezaka \& Piran (2017), Qin et al. (2018) investigated the strength and treatment of tides and they argued that WR stars may potentially be significantly spun up if placed in very close binaries with massive companions (e.g., immediate progenitors of $\mathrm{BH}-\mathrm{BH}$ mergers).

To determine the upper limit to the spins produced by tidal locking, we ignore the orbital evolution and assume that the entire star is tidally locked to the orbit (see the discussion below), meaning that it is rigidly rotating. The orbital period then provides the spin period of the WR star and implementing these spin periods into our stellar models, we can calculate the angular momentum in the star and use this angular momentum to obtain the spin period of the collapsed star, assumed to be the BH spin. Figure 5 shows the resultant black-hole spin magnitudes for our $Z=0.014$ and $Z=0.0004$ MESA models. For most of the models with orbital periods in the range of $P_{\text {orb }}=0.1-1.3 \mathrm{~d}$, the resultant $\mathrm{BH}$ spin magnitude can be fit by the exponential:

$a_{\text {spin }}=e^{-0.1\left(P_{\text {orb }} / P_{0}-1\right)^{1.1}}+0.125$,

where $P_{\text {orb }}$ is the orbital period in seconds and $P_{0}=4000 \mathrm{~s}$. For systems with orbital periods below $0.1 \mathrm{~d}$, the resultant $\mathrm{BH}$ spin is maximal. For systems with orbital periods longer than $\sim 1.3 \mathrm{~d}$, tidal locking takes longer than the duration of the relevant evolutionary phase and there is no significant spin-up (in which case the tidal spin-up is ignored). The fit with Eq. (15) is shown as the black curve in Fig. 5 .

We note that the proposal by Kushnir et al. (2016, 2017), Zaldarriaga et al. (2018), Hotokezaka \& Piran (2017), according to which every BH formed from a WR star subject to tidal interactions is spun-up to maximum $\left(a_{\text {spin }}=1\right)$, is subject to three caveats. First, for systems that are undergoing tidal synchronization it is assumed that the $\mathrm{BH}$ is formed with a maximal spin without close scrutiny of the WR star structure and its evolution under strong tidal interactions. Second, the scheme ignores the fact that WR wind mass-loss (depending on the metallicity of the WR star) may widen the system pushing it into a regime where synchronization is not maintained. Third, the tidal locking of the WR star pumps orbital energy into the WR star spin and thus causes the orbit to decay. This may lead to a merger of a WR star with its companion barring the formation of a $\mathrm{BH}-\mathrm{BH}$ binary. A detailed analysis of this complex interplay of stellar structure, stellar winds, and tidal interactions in the context of long Gamma Ray Bursts and BH-BH formation was presented by Detmers et al. (2008) and by Qin et al. (2018). In particular, Detmers et al. (2008) found that the majority of close BH-WR systems that are subject to strong tidal interactions either evolve to long periods (for high metallicity) or undergo a component merger before the $\mathrm{BH}-\mathrm{BH}$ formation (for low metallicity). On the other hand, Qin et al. (2018) found that most close BH-WR systems will increase their periods resulting in a wide range of secondary BH spins $\left(a_{\text {spin }}=0-1\right)$. In the light of these detailed calculations, our adopted simple model (see Eq. (15)) resembles the Qin et al. (2018) scheme and allows for a broad range of $\mathrm{BH}$ spin magnitudes if the WR star that is forming a $\mathrm{BH}$ is subject to strong tidal interactions. We should stress that we use this model only as an alternative to our standard assumption that the effect of tidal spin-up in close binaries on the $\mathrm{BH}$ natal spin magnitude

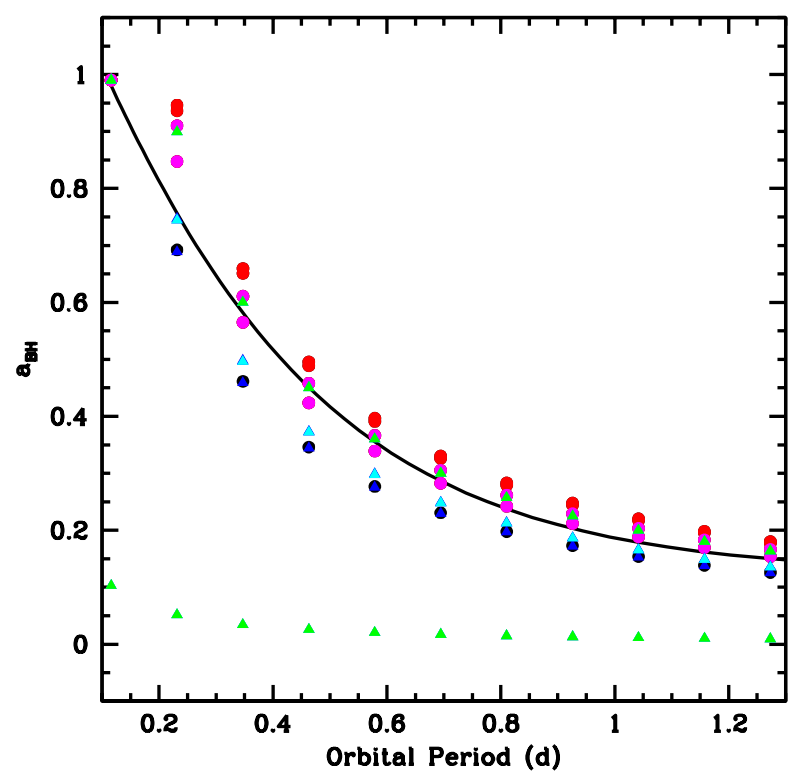

Fig. 5. Black hole spin magnitudes as a function of the orbital period for our $Z=0.014$ (circles) and $Z=0.0004$ (triangles) MESA models. The color coding corresponds to the black hole remnants with masses: $M_{\mathrm{BH}}<15 M_{\odot}$ (black/blue), $15 M_{\odot}<M_{\mathrm{BH}}<30 M_{\odot}($ cyan/red), $M_{\mathrm{BH}}>30 M_{\odot}$ (green/magenta). Binary systems with short orbital periods: $0.1-1.3 \mathrm{~d}$ and WR stars will produce BHs with broad range of spin magnitudes: $0.15-1$. Systems with orbital periods below $0.1 \mathrm{~d}$ form black holes from tidally locked WR stars, and the BH spins are maximal. Binaries with an orbital period above $1.3 \mathrm{~d}$ produce $\mathrm{BHs}$ with spins below 0.2 and typically are not tidally locked at all. A set of the most massive, lowest-metallicity stars have such dense cores that tidal spinup does not dramatically increase their angular momentum and these stars require orbital periods of less than $0.1 \mathrm{~d}$ to have spin values above 0.1 (lower set of green triangles). However, we ignore this fact, and let these cores to be spun-up to estimate the maximal effect of tidal interactions in our models (higher set of green triangles). The black curve shows our fit to the $\mathrm{BH}$ spin magnitude as a function of orbital period.

is ignored (as we use single stellar models to estimate natal $\mathrm{BH}$ spins; see Sect. 2.1).

Recently, Bavera et al. (2020) updated some of the Qin et al. (2018) calculations. We should note that these studies follow the angular momentum transport in stars only during the WR stage that will form the second-born $\mathrm{BH}$ in $\mathrm{BH}-\mathrm{BH}$ merger. Additionally, it is assumed that the first $\mathrm{BH}$ is born with zero spin $(a=0)$ and that the WR star that will form the second $\mathrm{BH}$ is also born spinless. Such simplifying assumptions allow to match LIGO/Virgo BH-BH effective spins which are mostly consistent with zero. However, it was only tested for initially non-spinning WR stars, and therefore did not take into account the fact that for inefficient angular momentum transport and high initial stellar rotation, WR stars can be born with high spins. Then for high-spinning WR stars and for inefficient angular momentum transport a significant fraction of the second-born BHs would have high spins independent of tidal interactions. This is demonstrated by our models that employ inefficient (Geneva) angular momentum transport, in which BHs (both first-born and secondborn) may have high spins (see Figs. 1 and 18).

\subsection{Cosmic star formation history and metallicity evolution}

Since 2016 (e.g., Belczynski et al. 2016a), we have been using the cosmic star formation density (SFRD) determinations from 


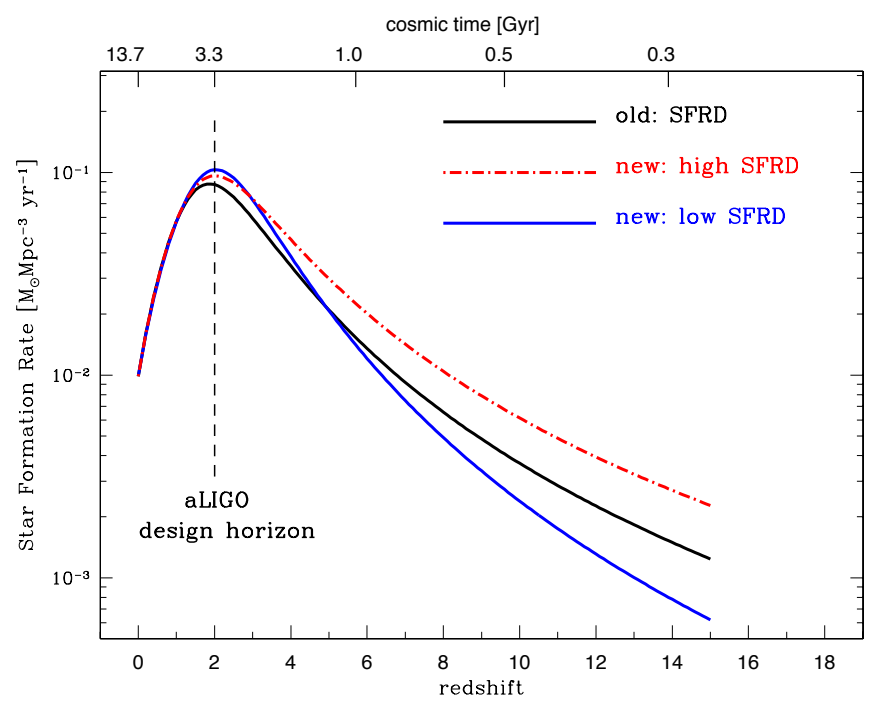

Fig. 6. Cosmic star formation histories adopted in our modeling (see Sect. 2.6 for details). We note how the different SFRDs agree at low redshifts $(z \lesssim 2)$, while they disagree by as much as a factor of three at earlier epochs.

Madau \& Dickinson (2014), which are based on a number of deep UV and infrared galaxy surveys. We refer to this SFRD as the "old SFRD" formula. Here, we adopt two best-fitting comoving SFRDs from Madau \& Fragos (2017), which update the previous formula by better reproducing a number of recent $4<z<10$ results:

$$
\operatorname{sfr}(z)=\mathcal{K}_{\mathrm{IMF}} 0.015 \frac{(1.0+z)^{2.6}}{1.0+((1.0+z) / 3.2)^{6.2}} M_{\odot} \mathrm{Mpc}^{-3} \mathrm{yr}^{-1}
$$

The correction factor $\mathcal{K}_{\mathrm{IMF}}$ adjusts the SFRD for the assumed IMF to the Salpeter IMF (i.e., $\mathcal{K}_{\text {IMF;Salpeter }}=1.0$ ). In this work we adopt a three component broken power-law Kroupa IMF with $\alpha_{3}=-2.35$ (see Appendix A.7 for details), for which the correction factor is $\mathcal{K}_{\text {IMF;Kroupa }} \approx 0.66$ (Madau \& Fragos 2017).

We refer to the SFRD given by Eq. (16) as to the "low SFRD", since it is based on a conversion from luminosity density to SFRD in which all published galaxy luminosity functions (LFs) have been conservatively integrated down to the same limiting luminosity of $0.03 L_{*}$, where $L_{*}$ is the characteristic luminosity of a Schechter function. For completeness, in the following we shall also provide results for a "high" model in which the comoving SFRD is increased at high redshifts to account for a steep faint end of the LF:

$\operatorname{sfr}(z)=\mathcal{K}_{\mathrm{IMF}} 0.015 \frac{(1.0+z)^{2.7}}{1.0+((1.0+z) / 3.0)^{5.35}} M_{\odot} \mathrm{Mpc}^{-3} \mathrm{yr}^{-1}$.

Hereafter, we refer to this formula as the "high SFRD". These expressions bracket uncertainties in the contribution to the early SFRD by galaxies fainter than $-16 \mathrm{mag}$, but agree at $z<2$. Our adopted SFRDs are shown in Fig. 6.

Our modeling of the metallicity of the star-forming gas has also been updated as follows. In previous calculations, we used the evolving mean cosmic metallicity $Z(z)$ from Madau $\&$ Dickinson (2014), which is the ratio between the total mass density of heavy elements produced over cosmic time and the cosmic baryon density. This quantity had to be increased by 0.5 dex to better account for various supernovae and GRB observations (Belczynski et al. 2016a). At each redshift, $z=0-15$, we further assumed that the distribution of $\log \left(Z / Z_{\odot}\right)$ was a Gaussian centered at the average metallicity and with a dispersion of $\sigma=0.5 \mathrm{dex}$. Stellar populations (Population I/II stars) with various metallicities were subsequently evolved, to check whether they produce NS-NS, BH-NS or BH-BH mergers detectable by LIGO/Virgo for a given instrument's sensitivity (see Appendix A.8).

This approach does not properly describe the metallicity evolution of the star-forming gas within galaxies, as only a small fraction of the baryons in the Universe are polluted with heavy elements and take part in the baryon cycle of galaxy evolution (e.g., Chruslinska et al. 2019). Our improved calculations follow Madau \& Fragos (2017). The gas-phase oxygen abundance is known to correlate strongly with the total stellar mass of starforming galaxies: this "mass-metallicity relation" (MZR) has been shown to extend down to low-luminosity galaxies with stellar masses $\sim 10^{6} M_{\odot}$ (Berg et al. 2012) and out to a redshift of 3.5 (Maiolino et al. 2008). Numerical modeling by Zahid et al. (2014) suggests that the MZR originates from a more fundamental, universal relationship between the metallicity and the stellarto-gas mass ratio that is followed by all galaxies as they evolve. We have assumed that the Zahid et al. (2014) MZR holds at all redshifts, and integrated this relation over the evolving galaxy stellar mass function at $0<z<7$ to compute a mean stellar mass-weighted gas-phase metallicity (see Madau \& Fragos 2017 for details and references) as:

$\log \left(Z / Z_{\odot}\right)=0.153-0.074 z^{1.34}$

As before, we assume the same distribution of $\log \left(Z / Z_{\odot}\right)$, but centered around this new average. We note that this is a simplification and this distribution may not be symmetric (Chruslinska et al. 2019). Recently, Chruslinska \& Nelemans (2019) contributed an observation-based distribution of the star formation rate density over metallicities and redshifts and discussed the uncertainty of this distribution. We note that the metallicity distribution used in our paper peaks at similar metallicities as in the high-metallicity extreme reported by those authors at $z \lesssim 3$ and at noticeably higher metallicities at $z>3$. However, at such high redshifts, the distribution is poorly constrained by current observations.

There is no consensus on the value of solar metallicity, and no value within the range of $Z_{\odot}=0.012-0.02$ can be rejected at the moment (Vagnozzi 2019). In our models we adopt either $Z_{\odot}=0.014$ or $Z_{\odot}=0.02$. A comparison between the adopted old and new mean gas-phase metallicities versus redshift is shown in Fig. 7.

\subsection{Calculations}

Our binary evolution calculations were performed with the upgraded population synthesis code StarTrack (Belczynski et al. 2002, 2008b). Improvements to the code include updates to the treatment of the common envelope (CE) evolution, compact object mass calculations including the effect of pair-instability pulsation supernovae and pair-instability supernovae, and new BH natal spin prescriptions (Sect. 2.1), among other upgrades (see Appendix A.7).

We considered fourteen different realizations (models) of our classical isolated binary evolution to test whether it is possible to form LIGO/Virgo BH-BH mergers with the observed rates, masses, and effective spins. The first two models correspond to our previous calculations with fallback-decreased, $\mathrm{BH}$ mass-dependent (M10), and high, mass-independent (M13) BH 


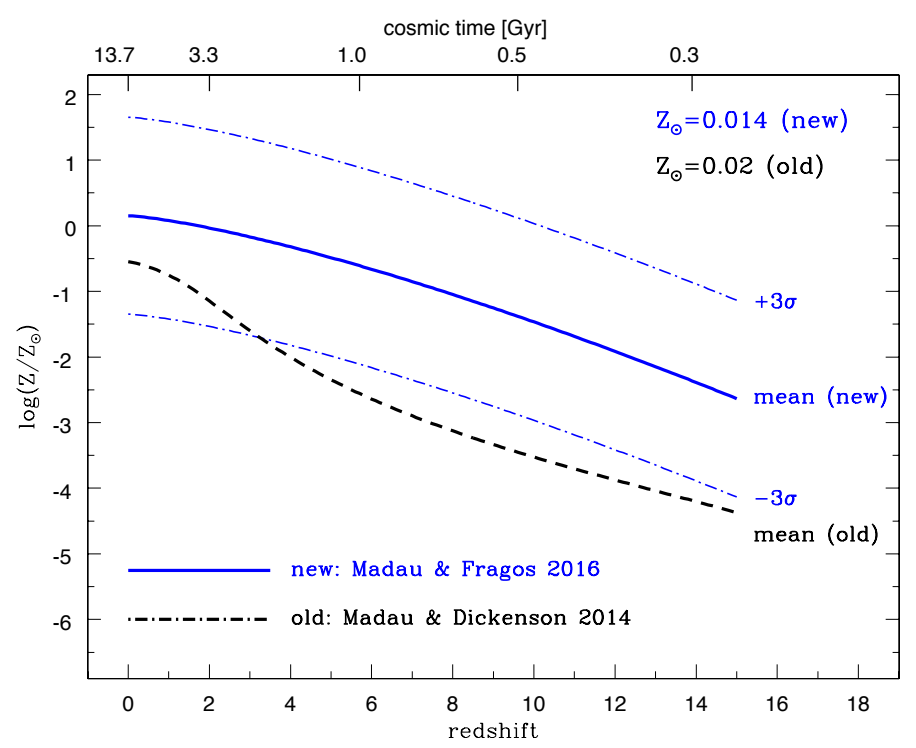

Fig. 7. Stellar mass-weighted gas-phase metallicity versus redshift, $Z(z)$. At every epoch, we adopted a Gaussian distribution of $\log \left(Z / Z_{\odot}\right)$, centered at the mean metallicity and with dispersion $\sigma=0.5$ dex. We note that the new metallicity for the star forming gas is noticeably higher than the mean cosmic metallicity adopted in the past (see Sect. 2.6).

natal kicks with input physics listed in Table 2 and detailed in (Belczynski et al. 2016a,c).

The next four models include different input physics on mass transfer, $\mathrm{BH}$ accretion in the $\mathrm{CE}$ phase, and the approximation of effects of stellar rotation on the BH mass (see Appendix A.7). These models include natal kicks which are fallback-decreased, BH mass-dependent (M20); small, BH mass-independent (M26; $\sigma=70 \mathrm{~km} \mathrm{~s}^{-1}$ ); intermediate, $\mathrm{BH}$ mass-independent (M25; $\sigma=130 \mathrm{~km} \mathrm{~s}^{-1}$ ); and high, BH mass-independent (M23; $\sigma=$ $265 \mathrm{~km} \mathrm{~s}^{-1}$ ).

The above six models employ $\mathrm{BH}$ natal spins adopted from the Geneva model with mild angular momentum transport in massive stars (Eq. (3)), SFRD and average metallicity evolution with redshift from Madau \& Dickinson (2014), and high value of solar metallicity $Z=0.02$. The next eight models represent our current update (2019) and tests of input physics.

M30 employs the rapid supernova engine model for NS/BH mass from Fryer et al. (2012) supplemented with weak PPSN (Eq. (9)), 1\% neutrino mass loss at the $\mathrm{BH}$ formation and $10 \%$ neutrino mass loss at the NS formation (Eq. (10)), fallbackdecreased, BH/NS mass-dependent, natal kicks (1D $\sigma=$ $265 \mathrm{~km} \mathrm{~s}^{-1}$ ), $50 \%$ non-conservative RLOF, 5\% Bondi-Hoyle accretion during $\mathrm{CE}$, inefficient accretion onto $\mathrm{BH} / \mathrm{NS}$ during stable mass transfer and capture from stellar winds (Sect. 2.4), BH natal spins from MESA (Eq. (4)), SFRD and average metallicity evolution from Madau \& Fragos (2017) (Eqs. (16) and (18)), and we adopt a low value for the solar metallicity $Z=0.014$. In this model, we do not take into account additional effects of rotation as is done in model M20, we use the standard stellar winds from Vink et al. (2001) with addition of LBV winds from Belczynski et al. (2010a), and we employ the initial binary parameters from Sana et al. (2012) as discussed in de Mink \& Belczynski (2015).

Models M33 and M35 are the same as model M30, but with mass independent NS/BH natal kicks (not affected by fallback): high natal kicks with 1D $\sigma=265 \mathrm{~km} \mathrm{~s}^{-1}$ (M33) and intermediate natal kicks with $1 \mathrm{D} \sigma=130 \mathrm{~km} \mathrm{~s}^{-1}$ (M35).
Table 2. Binary evolution models.

\begin{tabular}{|c|c|}
\hline Model & Main features \\
\hline M10 & $\begin{array}{l}2016 \text { standard input physics: } \\
\text { - Rapid SNa BH masses Fryer et al. (2012) } \\
\text { - With strong PPSN and with PSN } \\
\text { - } 10 \% \text { neutrino mass loss at BH/NS formation } \\
\text { - Low-to-no BH natal kicks (set by fallback) } \\
\text { - High NS kicks: } \sigma=265 \mathrm{~km} \mathrm{~s}^{-1} \text { with fallback } \\
\text { - 50\% non-conservative RLOF } \\
\text { - } 10 \% \text { Bondi-Hoyle accretion onto NS/BH in CE } \\
\text { - Efficient accretion onto BH in stable MT/winds } \\
\text { - No effects of rotation on stellar evolution }{ }^{(a)} \\
\text { - Initial binary parameters: Sana et al. }(2012) \\
\text { - Massive star winds: Vink et al. }(2001)+\text { LBV }^{(b)} \\
\text { - BH spins: Geneva models (Eq. }(3)) \\
\text { - SFRD }(z) \text { and } Z(z): \text { Madau \& Dickinson }(2014) \\
\text { - Solar metallicity: } Z_{\odot}=0.02\end{array}$ \\
\hline M13 & $\begin{array}{l}\text { As in M10, but with: } \\
\text { - High BH/NS natal kicks: } \sigma=265 \mathrm{~km} \mathrm{~s}^{-1}\end{array}$ \\
\hline M20 & $\begin{array}{l}\text { Modified input physics, as in M10, but with: } \\
-80 \% \text { non-conservative RLOF (Appendix A.7) } \\
-5 \% \text { Bondi-Hoyle accretion onto NS/BH in CE } \\
\text { - Rotation increases CO core mass (by } 20 \% \text { ) }\end{array}$ \\
\hline M26 & $\begin{array}{l}\text { As in M20, but with: } \\
- \text { Small BH/NS natal kicks: } \sigma=70 \mathrm{~km} \mathrm{~s}^{-1}\end{array}$ \\
\hline M25 & $\begin{array}{l}\text { As in M20, but with: } \\
\text { - Intermediate BH/NS natal kicks: } \sigma=130 \mathrm{~km} \mathrm{~s}^{-1}\end{array}$ \\
\hline M23 & $\begin{array}{l}\text { As in M20, but with: } \\
\text { - High BH/NS natal kicks: } \sigma=265 \mathrm{~km} \mathrm{~s}^{-1}\end{array}$ \\
\hline M30 & $\begin{array}{l}2019 \text { standard input physics: } \\
\text { - Rapid SNa BH masses Fryer et al. (2012) } \\
\text { - With weak PPSN and with PSN } \\
\text { - } 1 \% \text { neutrino mass loss at BH formation } \\
\text { - } 10 \% \text { neutrino mass loss at NS formation } \\
\text { - Low-to-no BH natal kicks (set by fallback) } \\
\text { - High NS kicks: } \sigma=265 \mathrm{~km} \mathrm{~s}^{-1} \text { with fallback } \\
\text { - 50\% non-conservative RLOF } \\
\text { - 5\% Bondi-Hoyle accretion onto NS/BH in CE } \\
\text { - Inefficient accretion onto BH in stable MT/winds } \\
\text { - No effects of rotation on stellar evolution }{ }^{(a)} \\
\text { - Initial binary parameters: Sana et al. }(2012) \\
\text { - Massive star winds: Vink et al. }(2001)+\mathrm{LBV}{ }^{(b)} \\
\text { - BH spins: MESA models (Eq. (4)) } \\
\text { - SFRD }(z) \text { and } Z(z): \text { Madau \& Fragos }(2017) \\
\text { - Solar metallicity: } Z_{\odot}=0.014\end{array}$ \\
\hline M33 & $\begin{array}{l}\text { As in M30, but with: } \\
\text { - High BH/NS natal kicks: } \sigma=265 \mathrm{~km} \mathrm{~s}^{-1}\end{array}$ \\
\hline M35 & $\begin{array}{l}\text { As in M30, but with: } \\
\text { - Intermediate BH/NS natal kicks: } \sigma=130 \mathrm{~km} \mathrm{~s}^{-1}\end{array}$ \\
\hline M40 & $\begin{array}{l}\text { As in M30, but with: } \\
\text { - BH spins: Fuller model (Eq. (5)) }\end{array}$ \\
\hline M43 & $\begin{array}{l}\text { As in M40, but with: } \\
\text { - High BH/NS natal kicks: } \sigma=265 \mathrm{~km} \mathrm{~s}^{-1}\end{array}$ \\
\hline M50 & $\begin{array}{l}\text { As in M30, but with: } \\
-30 \% \text { of wind mass loss rates for all stars }\end{array}$ \\
\hline M60 & $\begin{array}{l}\text { As in M30, but with: } \\
\text { - Strong PPSN, } 10 \% \text { neutrino mass loss for BH/NS }\end{array}$ \\
\hline M70 & $\begin{array}{l}\text { As in M30, but with: } \\
\text { - Moderate PPSN }\end{array}$ \\
\hline
\end{tabular}

Notes. ${ }^{(a)}$ Stellar spins are followed (tides, magnetic braking, change of inertia), but rotation does not alter the star properties (He/CO core mass). ${ }^{(b)}$ Luminous Blue Variable winds: $1.5 \times 10^{-4} M_{\odot} \mathrm{yr}^{-1}$.

Model M40 is the same as model M30, but with a different model for BH spins: the Fuller model (Eq. (5)). Model M43 is the same as model M40, but with mass-independent NS/BH natal kicks with 1D $\sigma=265 \mathrm{~km} \mathrm{~s}^{-1}$. 
Model M50 is the same as model M30, but with mass loss rates reduced to $30 \%$ for all the stars (see Appendix A.7 for a justification).

Model M60 is the same as model M30, but with strong PPSN (Eq. (7)) with $10 \%$ neutrino mass loss for both BH and NS.

Model M70 is the same as model M30, but with moderate PPSN (Eq. (8)).

Models that allow CE with HG donors are marked as submodels MXX.A, while models that do not allow CE with HG donors are marked as as MXX.B (Belczynski et al. 2007).

In all models, we assume $\mathrm{BH}$ natal kicks to be randomly oriented, thus generating $\mathrm{BH}$ spin misalignments with respect to the orbital angular momentum. We note that our models are by no means exhaustive in terms of probing the evolutionary uncertainties. However, they allow us to test the key parameters that set $\mathrm{BH}$ spin magnitudes and misalignments, and thus the effective spin: angular momentum transport in massive stars, accretion onto the $\mathrm{BH}$ and its progenitor, tidal interactions and $\mathrm{BH}$ natal kicks. Table 2 gives an overview of the models, and in Appendix A.7 we describe the details of our binary population synthesis calculations.

The StarTrack population synthesis code is used to generate populations of BH-BH/BH-NS/NS-NS systems. The star's initial properties (mass and metallicity) are used to calculate stellar evolution. Binary interactions (mass gain and loss in RLOF and $\mathrm{CE}$ events) are taken into account through rejuvenation and de-rejuvenation in estimating the final stellar properties (mass and $\mathrm{CO}$ core mass) at the time of core-collapse, which are then used to obtain the NS/BH natal mass (see Appendix A.3). In binary calculations, we use non-rotating stellar models (Hurley et al. 2000). We record whether any of the binary components accreted significant amounts of mass ( $\gtrsim 10 \%$ of its own mass). If accretion occurred during main sequence and if the accreting star was of low metallicity $(Z<0.002)$, then we assume in models M20, M23, M25, and M26 that the star will produce a more massive CO core (greater by $20 \%$ ) and, thus, a more massive $\mathrm{NS} / \mathrm{BH}$ to mimic the effects of increased mixing due to rapid rotation induced by accretion (see Appendix A.7).

At $\mathrm{BH}$ formation we use single stellar models to estimate the $\mathrm{BH}$ natal spin magnitude through the $\mathrm{CO}$-core mass-spin relations proposed in Sect. 2.1. In these estimates, we assume that a star with a given $\mathrm{CO}$ core mass (as estimated from a binary evolution) forms a $\mathrm{BH}$ with spin given by single stellar models. This scheme ignores the effects of mass accretion on the stellar spin of the BH progenitor that may increase the BH's natal spin. Obviously, this is far from perfect, and stellar rotation models will need to be fully integrated into binary population synthesis in the future. However, this is impossible at the moment due to the lack of stellar models with consistent input physics that would appropriately sample mass, metallicity and rotation for massive stars and naked stellar cores (as stellar cores are much more often exposed in binary evolution than is single star evolution).

We note also that we use moderately high initial stellar spins that assume $40 \%$ of break-up velocity in single stellar models. Depending on a mass and stellar structure of a model, this gives a range of $250-450 \mathrm{~km} \mathrm{~s}^{-1}$ initial rotation speeds at the equator (see Tables A.1 and A.2). For comparison, the observed spins of massive stars show a bimodal distribution (Ramírez-Agudelo et al. 2013), with one large peak at $\sim 100 \mathrm{~km} \mathrm{~s}^{-1}$ and another small peak at $\sim 400 \mathrm{~km} \mathrm{~s}^{-1}$.

A first-born BH can accrete mass from its unevolved companion either during the RLOF/CE stages or from the companion's winds. These effects are included in our calculations, and the $\mathrm{BH}$ spin is increased accordingly. The second-born BH preserves its natal spin as it does not accrete mass. However, we note that we allow for the possibility of the tidal spin up of $\mathrm{BH}$ progenitors and allow for significant increase of the $\mathrm{BH}$ spin as compared to $\mathrm{BH}$ spins that result from single stellar models (Sect. 2.5).

In all cases, we assume that the stellar spins are initially aligned $\left(\Theta_{1}=\Theta_{2}=0\right)$ with the orbital angular momentum of the main-sequence star binary. At BH formation we estimate the spin vector misalignment due to natal kicks. We allow for $\mathrm{BH}$ spin realignment neither during the mass transfer phases, nor by tidal interaction between the stars in a binary. We note that with this approach we may be overestimating the $\mathrm{BH}$ spin misalignment, but only for models with high to moderate BH natal kicks (see Sect. 2.2 for details).

\section{Results}

We estimated the double compact object merger rate densities, merger detection rates, merger masses, and $\mathrm{BH}-\mathrm{BH}$ effective spins using the methods presented in Belczynski et al. (2016b,a) along with updates and revisions presented in the present study (see Sect. 2). In the following sections, we discuss some particular properties of our models. In our study, we do not exhaust the information that can be extracted from our models. Focusing on BH-BH mergers, we instead show some particular examples of what can be obtained with population synthesis modeling in context of the LIGO/Virgo sources. For now, we compare our models to the LIGO/Virgo observations showing that some models fit the data better than other, but only in terms of the observed allowed ranges of rates, masses, and effective spins. We note that we do not yet attempt to match particular distributions' shapes (for $\mathrm{BH}$ masses and effective spins) or the rate of increase of merger rates with redshift. However, anyone interested in such comparisons can easily perform them on our models as we make them publicly accessible through our website ${ }^{3,4}$.

\subsection{Binary evolution of $\mathrm{BH}-\mathrm{BH}$ mergers}

In this section, we present several examples of binary evolution leading to the formation of $\mathrm{BH}-\mathrm{BH}$ mergers. In the framework of the Geneva model of angular momentum transport, it is challenging to explain mergers with very low effective spin parameters as lots of BHs are formed with high or moderate spin magnitudes (see Fig. 1). Yet, it is not impossible. Therefore, we show examples of evolution that can lead to the formation of merger resembling GW170104, which has one of the lowest measured effective spin parameters: $-0.24<\chi_{\text {eff }}<0.13$ (90\% confidence limits). In the framework of MESA angular momentum transport, it is challenging to explain mergers with moderate and high effective spin parameters as lots of BHs are formed with low spin magnitudes (see Fig. 2). Yet, we show that we can form mergers that are consistent even with GW170729, which is the merger that has the highest effective spin yet measured: $0.11<\chi_{\text {eff }}<0.57$ (90\% confidence limits).

\subsubsection{Case of GW170104}

Here we present a proof-of-principle scenario demonstrating that isolated binary evolution with the Geneva model of angu-

\footnotetext{
3 wWw. syntheticuniverse.org

4 The models will appear on this website at the moment of the astro-ph submission.
} 
lar momentum transport can form a $\mathrm{BH}-\mathrm{BH}$ merger with $\mathrm{BH}$ masses and effective spin compatible with LIGO's observation of GW170104; in particular, given its very low effective spin.

Within our models with the Geneva angular momentum transport (M10, M13, M20, M23, M25, M26), we search for systems with BH masses and effective spins within LIGO's $90 \%$ credible limits: $25.4<M_{\mathrm{BH} 1}<38.2 M_{\odot}, 15.6<M_{\mathrm{BH} 2}<$ $25.0 M_{\odot},-0.24<\chi_{\text {eff }}<0.13$. The upper bound on $\chi_{\text {eff }}$ may actually be as high as $\chi_{\text {eff }} \approx 0.2$ (Appendix A.5). For example, within model M20, it is indeed possible to form a BH-BH merger resembling GW170104: $M_{\mathrm{BH} 1}=33.3 M_{\odot}, M_{\mathrm{BH} 2}=24.7 M_{\odot}$, $\chi_{\text {eff }}=0.09$. The evolutionary history of such a merger is presented in Fig. 8. We note that model M20 is rather conservative regarding assumptions on natal kicks, which are strongly suppressed by the fallback material. Massive $\mathrm{BH}$ spins are mostly aligned with the binary angular momentum $\left(\cos \Theta_{1}=\cos \Theta_{2}=\right.$ 1), thus maximizing the value of the effective spin. For all the other models (with the exception of M10) the BH spins will tend to be misaligned, decreasing the value of the effective spin and making it even easier to produce systems with low effective spin values (as observed in GW170104).

Although the $\mathrm{BH}$ spin can be modified by accretion from a binary companion, the amount of matter accreted in our calculations is very modest, and the accretion-induced spin-up of BHs is not significant. In the example shown in Fig. 8, the first-born massive $\mathrm{BH}$ forms with no spin $\left(a_{\text {spin } 1}=0\right)$, then it accretes very little mass from its MS companion wind increasing its spin only to $a_{\text {spin1 }}=0.02$. Most of the accretion occurs during the $\mathrm{CE}$ phase $\left(0.4 M_{\odot}\right)$ and the $\mathrm{BH}$ increases its spin to $a_{\text {spin } 1}=0.05$. Finally, the BH accretes very little mass from its Wolf-Rayet star companion wind, increasing its spin to its final value of $a_{\text {spin1 }}=0.053$. The second-born BH forms with a natal spin of $a_{\text {spin1 }}=0.14$ and it is not spun up, as it does not accrete any material. In other words, we predict that LIGO/Virgo observations of $\mathrm{BH}-\mathrm{BH}$ mergers will probe the natal $\mathrm{BH}$ spin distribution, up to evolutionary effects of order 0.05 in dimensionless spin.

We also note that this particular system is not subject to a potential WR-star tidal spin-up in a BH-WR binary (the last evolutionary stage before the $\mathrm{BH}-\mathrm{BH}$ formation). The system separation at this stage $\left(a \approx 35 R_{\odot}\right)$ is too large for the tides to effectively spin-up WR star $\left(P_{\text {orb }}>1.3 \mathrm{~d}\right.$, see Sect. 2.5$)$. The full details of this evolutionary example are given in Appendix A.4.

\subsubsection{Case of GW170729}

In a subset of our binary evolution models M30.A/B, M33.A/B, M35.A/B, M50.A/B, M60.A/B, and M70.A/B, the natal BH spins are obtained from stellar models calculated with the MESA code under the assumption of efficient angular momentum transport in the stellar interiors (see Sect. 2.1 and Fig. 2). In this framework, the initial BH spins are always small $\left(a_{\text {spin }} \lesssim 0.15\right)$, mostly independent of the progenitor mass and metallicity. Small natal $\mathrm{BH}$ spin values can, in principle, be increased during further evolution as result of mass accretion. However, in the isolated binary evolution channel for $\mathrm{BH}-\mathrm{BH}$ mergers, the first formed $\mathrm{BH}$ can only accrete mass during the $\mathrm{CE}$ inspiral and through accretion of the wind from its stellar companion. We find that in our simulations neither of those two processes leads to a significant increase of the $\mathrm{BH}$ spin which is primarily the consequence of: (i) inefficient (5-10\%) Bondi-Hoyle accretion rate onto $\mathrm{BH}$ in the short-lived $\mathrm{CE}$ phase (e.g., MacLeod et al. 2017) and (ii) small wind mass loss rates from low metallicity stars (Vink et al. 2001). This means that the small natal BH spins in the framework of efficient angular momentum transport

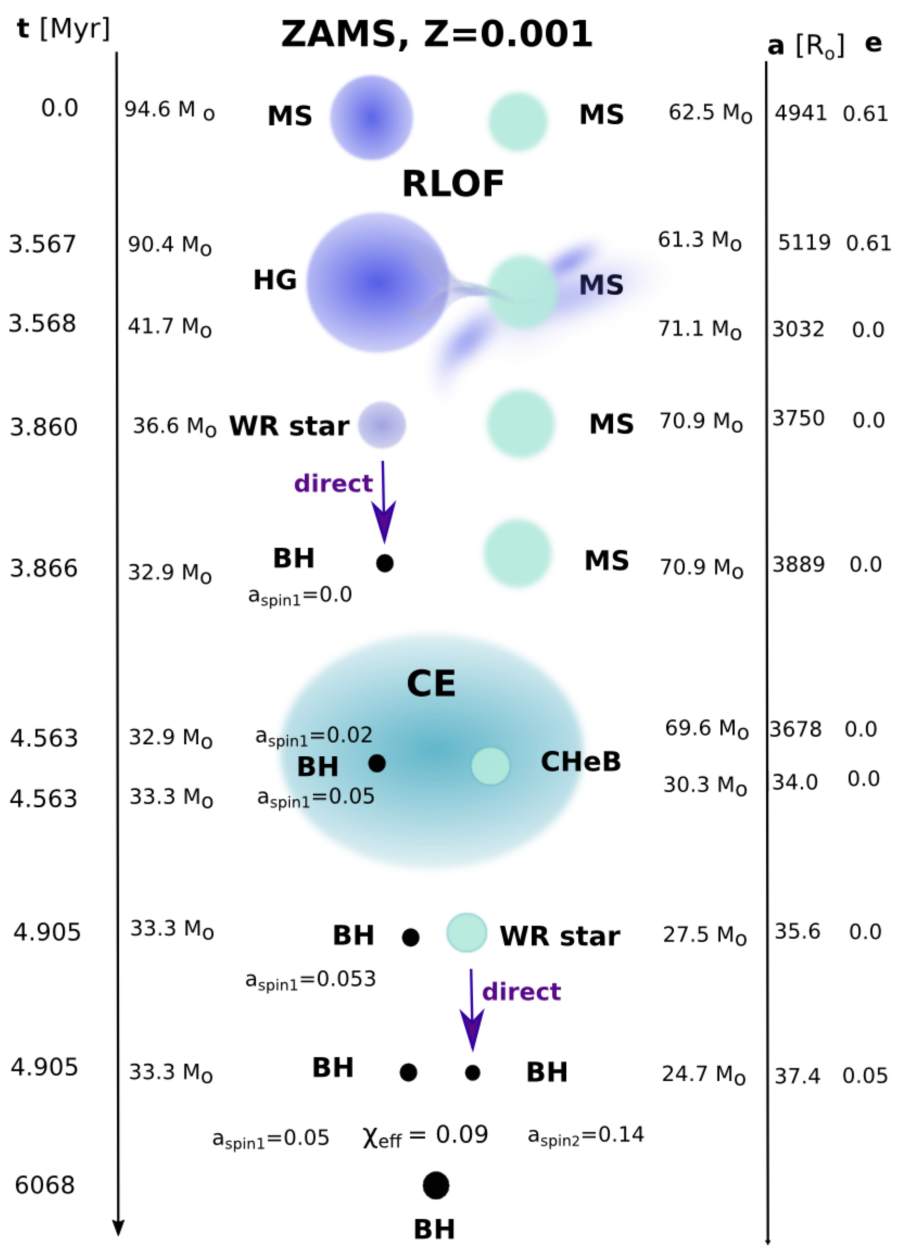

Fig. 8. Example of a possible route leading to the formation of a $\mathrm{BH}$ BH merger similar to GW170104. This example follows the classical isolated binary evolution channel. In this model (M20.B) we employ the Geneva BH natal spins, assume that massive $\mathrm{BHs}$ do not receive natal kicks and that their spins are aligned with the binary angular momentum $\left(\Theta_{1}=\Theta_{2}=0^{\circ}\right)$, producing an upper limit on the effective spin parameter $\left(\chi_{\text {eff }}\right)$. Yet, this system has $\chi_{\text {eff }}=0.09$, within LIGO's $90 \%$ credible limits for GW170104 [-0.24:0.13]. Both BH masses are also within the limits: $M_{\mathrm{BH} 1}=31.0 M_{\odot}[25.4: 38.2]$ and $M_{\mathrm{BH} 2}=20.1 M_{\odot}[15.6: 25.0]$.

in stellar interiors do result in small effective spin values of $\mathrm{BH}$ BH mergers (typically $\chi_{\text {eff }} \lesssim 0.25$ ).

Here we present a proof-of-principle scenario demonstrating that even the $\mathrm{BH}-\mathrm{BH}$ merger event with the largest effective spin value reported to date $\left(\mathrm{GW} 170729, \chi_{\text {eff }}=0.36_{-0.25}^{+0.21}\right)$ can be reconstructed in the isolated-binary evolution channel with small natal BH spin values given by Eq. (4). Within our models with efficient (MESA) angular momentum transport we search for systems with BH masses and effective spin within LIGO's 90\% credible limits for GW170729: $40.4<M_{\mathrm{BH} 1}<67.2 M_{\odot}$, $24.2<M_{\mathrm{BH} 2}<43.4 M_{\odot}, 0.11<\chi_{\mathrm{eff}}<0.57$. For example, within the model M30.B it is indeed possible to form a $\mathrm{BH}-$ BH merger resembling GW170729: $M_{\mathrm{BH} 1}=55.0 M_{\odot}, M_{\mathrm{BH} 2}=$ $32.8 M_{\odot}, \chi_{\text {eff }}=0.137$. The evolutionary history of such a merger is presented in Fig. 9. Both the BHs where formed in direct collapse with small but non-zero natal spins $\left(a_{\text {spin1 }}=0.093\right.$ and $a_{\text {spin2 }}=0.073$ ). For reasons discussed above, the $\mathrm{BH}$ formed first accreted only a very modest amount of mass during binary evolution $\left(<1.0 M_{\odot}\right)$, which led to an increase of its spin to $a_{\text {spin1 }}=0.176$. The final effective spin of the merging $\mathrm{BH}-\mathrm{BH}$ 


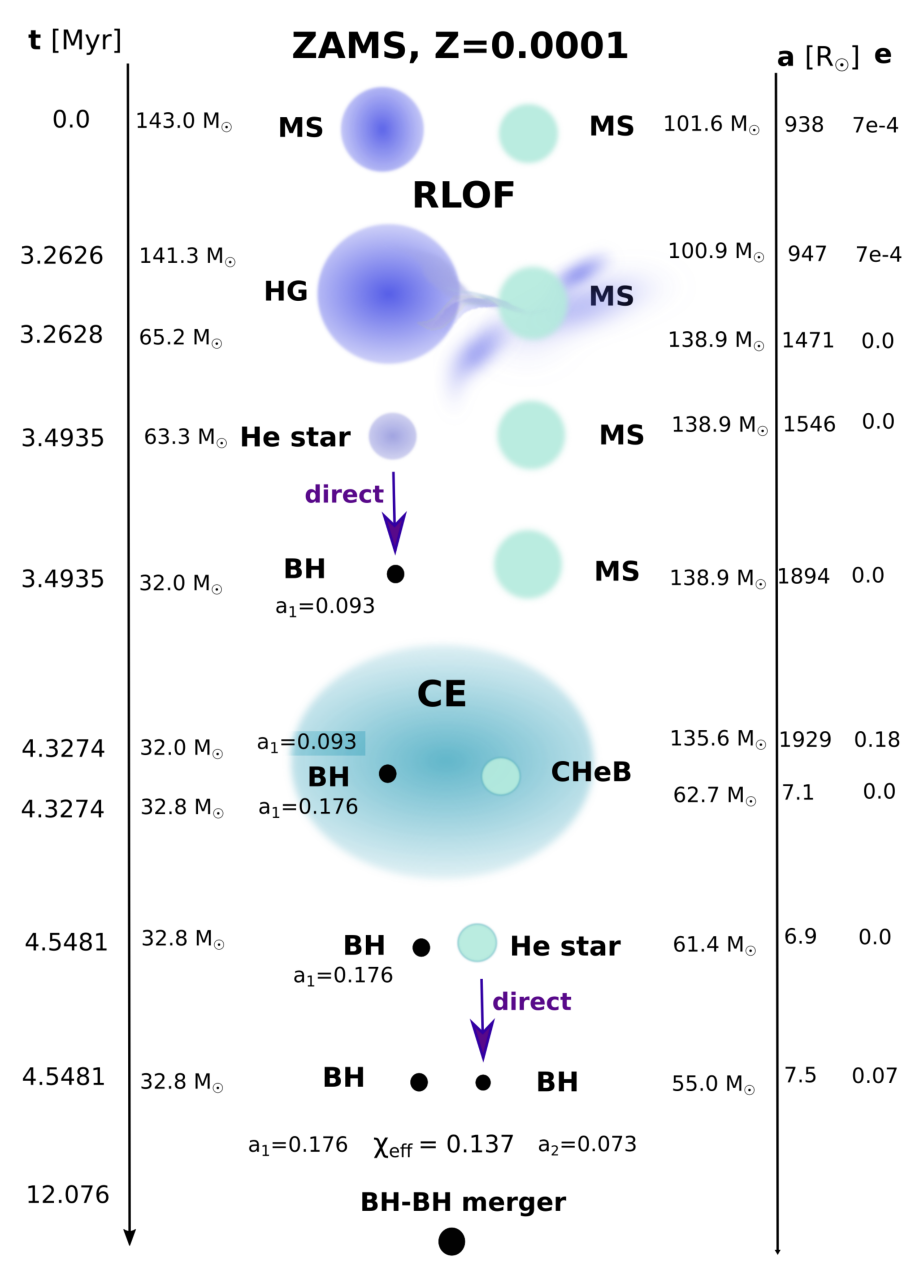

Fig. 9. Example of a possible route leading to the formation of a $\mathrm{BH}-$ BH merger similar to GW170729. This example follows the classical isolated binary evolution channel. In this model (M30.B) we employ the MESA BH natal spins, assume that massive $\mathrm{BHs}$ do not receive natal kicks and that their spins are aligned with the binary angular momentum $\left(\Theta_{1}=\Theta_{2}=0^{\circ}\right)$, producing an upper limit on the effective spin parameter $\left(\chi_{\text {eff }}\right)$. We note that given the small binary separation at the BH-WR stage $\left(a \approx 7 R_{\odot}\right)$, the WR star is most likely going to become tidally synchronized (see Sect. 2.5). If we assume that the rapidly rotating WR star collapses into rapidly spinning $\mathrm{BH}\left(a_{\text {spin2 }}=1\right)$ then the effective spin of the presented system would increase from $\chi_{\mathrm{eff}}=0.137$ (no tidal spin-up; presented in the figure) to $\chi_{\text {eff }}=0.484$ (full tidal spin-up). Both values are within the LIGO/Virgo 90\% credible limits for GW170729 [0.11:0.57]. Both BH masses are also within the limits: $M_{\mathrm{BH} 1}=50.6 M_{\odot}$ [40.4:67.2] and $M_{\mathrm{BH} 2}=34.3 M_{\odot}[24.2: 43.4]$.

system is $\chi_{\text {eff }}=0.137$, and thus it is in (marginal) agreement with the $90 \%$ credible limits for GW170729.

It should be noted that the spin value for the secondary $\mathrm{BH}$ presented in the above example (Fig. 9; $a_{\text {spin2 }}=0.073$ ) is most likely underestimated because this example neglects the potential tidal spin-up of the secondary WR star (direct progenitor of the second $\mathrm{BH}$ ) during the BH-WR stage (see Sect. 2.5). Since the orbital separation during the BH-WR phase is very small $\left(a \approx 7 R_{\odot}\right)$, tidal interactions are expected to be efficient in spinning-up the WR star. In fact, in the case of this system the timescale of WR tidal synchronization as approximated by Zaldarriaga et al. (2018, see our Eq. (15)) is only $t_{\text {sync }} \approx 11 \mathrm{yr}$, which is a few orders of magnitude shorter than the duration of BH-WR stage $\left(\sim 2 \times 10^{5} \mathrm{yr}\right)$. Thus, the secondary WR star has most likely become fully synchronized with the orbit by the time it collapsed to form the second BH. If we assume that such a rapidly rotating WR star collapses into an also rapidly spinning $\mathrm{BH}\left(a_{\text {spin2 }}=1.0\right)$ then the effective spin of the BH-BH merger in Fig. 9 would become $\chi_{\text {eff }}=0.484$, still well within the $90 \%$ credible limits for GW170729. The large range of $\chi_{\text {eff }}$ values that spans from 0.137 to 0.484 encloses all the possible results of the uncertain process of tidal spin-up in BH-WR binaries and its effect on the secondary BH spin. As a word of caution, we note that the simple fact that a particular BH-BH system can be reproduced in a given binary evolution channel does not in itself guarantee consistency between population synthesis results and observations. Such consistency can only be tested by comparing the entire populations. We discuss the distributions of $\mathrm{BH}-\mathrm{BH}$ mergers parameters obtained in our simulation in the following sections.

\subsection{Merger rate density and detection rate}

Table 3 summarize our predictions for the local (redshift $z \sim 0$ ) merger rate density, the detection rate for LIGO/Virgo's midhigh sensitivity curve (that may be taken as an approximation of $\mathrm{O} 3$ observing run) along with the maximum horizon redshift for the best located and oriented source in a given merger type category: NS-NS, BH-NS, BH-BH.

The predicted $\mathrm{BH}-\mathrm{BH}$ merger rate densities vary between 1.24 and $1368 \mathrm{Gpc}^{-3} \mathrm{yr}^{-1}$ across our models. A number of models (M13.A, M23.A/B, M25.B, M26.B, M30.B, M33.A, M40.B, M43.A, M50.B, M60.B, M70.B) produce rates within the allowable range determined by the first $10 \mathrm{LIGO} /$ Virgo detections (9.7-101 Gpc $\mathrm{Gr}^{-3} \mathrm{yr}^{-1}$ : Abbott et al. 2019b). The predicted NSNS merger rate densities vary between 49.3 and $524 \mathrm{Gpc}^{-3} \mathrm{yr}^{-1}$ for the tested models. A number of models (M10.A,M13.A, M20.A, M23.A, M25.A, M26.A, M30.A/B, M33.A, M35.A/B, M40.A/B, M43.A, M50.A, M60.A/B, M70.A/B) produce rates within allowable range determined by the first LIGO/Virgo detection (110-3840 $\mathrm{Gpc}^{-3} \mathrm{yr}^{-1}$ : Abbott et al. 2019b). The predicted BH-NS merger rate densities vary between 0.48 and $297 \mathrm{Gpc}^{-3} \mathrm{yr}^{-1}$ for the tested models. So far, all the models produce rates within an upper limit determined by the non-detection in the O1/O2 LIGO/Virgo runs $\left(<610 \mathrm{Gpc}^{-3} \mathrm{yr}^{-1}\right.$ : Abbott et al. 2019b).

Figure 10 shows the intrinsic $\mathrm{BH}-\mathrm{BH}$ merger rate density (not weighted by LIGO/Virgo detection probability) evolution with redshift for models M10.B and M30.B that employ different star formation rate and cosmic evolution of metallicity. We note that model M10.B which employs $Z(z)$ measured from stars generates higher (by factor of $\sim 5$ at low redshifts $z<2$ ) rates than the model M30.B that employs $Z(z)$ as measured by the metallicity of star forming gas at any given redshift. The choice of $Z(z)$ is one of the most important factors affecting local (low z) merger rate densities for BH-BH mergers. This is because with decreasing metallicity we expect (i) an increase of the $\mathrm{BH}$ mass (Belczynski et al. 2010a) and (ii) an increase of the BH$\mathrm{BH}$ merger formation efficiency per unit mass (Belczynski et al. 2010 b). Therefore, models with lower $Z(z)$ result in higher $\mathrm{BH}-$ BH merger rates.

We note that the difference in $\operatorname{SFRD}(z)$ between the two models does not affect significantly the rates as the differences in star formation are relatively small (see Fig. 6). We also note that for the two updated (new) models of the $\operatorname{SFRD}(z)$, there is virtually no difference in the $\mathrm{BH}-\mathrm{BH}$ merger rates at low redshifts $(z<2)$, while differences ( $\sim$ factor of a few) begin to appear only at higher redshifts. For our updated models, we use a $\operatorname{SFRD}(z)$ 
Table 3. Local merger rate densities and LIGO/Virgo detection rates.

\begin{tabular}{|c|c|c|c|c|}
\hline Model & Merger & $\begin{array}{c}\text { Rate density }{ }^{(a)} \\
{\left[\mathrm{Gpc}^{-3} \mathrm{yr}^{-1}\right]}\end{array}$ & $\begin{array}{c}\text { Detection rate } \\
{\left[\mathrm{yr}^{-1}\right]}\end{array}$ & $z_{\text {hor }}{ }^{(c)}$ \\
\hline \multirow[t]{3}{*}{ M10.B } & NS-NS & 65.2 & 0.289 & 0.081 \\
\hline & BH-NS & 28.8 & 2.419 & 0.404 \\
\hline & BH-BH & 274 & 536.2 & 0.961 \\
\hline \multirow[t]{3}{*}{ M13.B } & NS-NS & 49.3 & 0.226 & 0.082 \\
\hline & BH-NS & 2.43 & 0.246 & 0.397 \\
\hline & ВH-BH & 8.63 & 16.60 & 1.031 \\
\hline \multirow[t]{3}{*}{ M20.B } & NS-NS & 84.3 & 0.358 & 0.081 \\
\hline & BH-NS & 96.6 & 6.525 & 0.405 \\
\hline & BH-BH & 469 & 783.7 & 0.955 \\
\hline \multirow{3}{*}{ M26.B } & NS-NS & 107 & 0.453 & 0.082 \\
\hline & BH-NS & 42.4 & 2.880 & 0.401 \\
\hline & BH-BH & 132 & 192.8 & 0.958 \\
\hline \multirow{3}{*}{ M25.B } & NS-NS & 105 & 0.447 & 0.081 \\
\hline & BH-NS & 17.2 & 1.486 & 0.426 \\
\hline & BH-BH & 62.8 & 105.4 & 0.953 \\
\hline \multirow{3}{*}{ M23.B } & NS-NS & 53.0 & 0.225 & 0.080 \\
\hline & BH-NS & 4.17 & 0.337 & 0.397 \\
\hline & ВН-BH & 16.6 & 26.29 & 1.024 \\
\hline \multirow{3}{*}{ M30.B } & NS-NS & 122 & 0.514 & 0.081 \\
\hline & BH-NS & 11.1 & 0.800 & 0.433 \\
\hline & ВН-BH & 43.7 & 50.95 & 1.152 \\
\hline \multirow[t]{3}{*}{ M35.B } & NS-NS & 143 & 0.608 & 0.081 \\
\hline & BH-NS & 3.10 & 0.206 & 0.444 \\
\hline & BH-BH & 4.69 & 6.627 & 1.152 \\
\hline \multirow[t]{3}{*}{ M33.B } & NS-NS & 79.6 & 0.333 & 0.080 \\
\hline & BH-NS & 0.48 & 0.031 & 0.392 \\
\hline & BH-BH & 1.37 & 1.714 & 1.147 \\
\hline \multirow[t]{3}{*}{ M40.B } & NS-NS & 122 & 0.508 & 0.081 \\
\hline & BH-NS & 10.2 & 0.752 & 0.391 \\
\hline & BH-BH & 39.2 & 42.98 & 1.152 \\
\hline \multirow{3}{*}{ M43.B } & NS-NS & 76.7 & 0.319 & 0.080 \\
\hline & BH-NS & 0.61 & 0.044 & 0.381 \\
\hline & BH-BH & 1.24 & 1.578 & 1.150 \\
\hline \multirow[t]{3}{*}{ M50.B } & NS-NS & 93.2 & 0.395 & 0.081 \\
\hline & BH-NS & 8.54 & 0.702 & 0.489 \\
\hline & ВН-BH & 64.2 & 133.4 & 1.153 \\
\hline \multirow[t]{3}{*}{ M60.B } & NS-NS & 118 & 0.499 & 0.080 \\
\hline & BH-NS & 12.1 & 0.817 & 0.363 \\
\hline & BH-BH & 46.2 & 41.88 & 0.949 \\
\hline \multirow[t]{3}{*}{ M70.B } & NS-NS & 120 & 0.505 & 0.081 \\
\hline & BH-NS & 10.7 & 0.765 & 0.396 \\
\hline & ВН-BH & 43.9 & 51.37 & 1.107 \\
\hline \multirow[t]{3}{*}{ M10.A } & NS-NS & 230 & 1.022 & 0.083 \\
\hline & BH-NS & 99.6 & 6.289 & 0.404 \\
\hline & BH-BH & 1368 & 3464 & 0.973 \\
\hline \multirow[t]{3}{*}{ M13.A } & NS-NS & 193 & 0.858 & 0.083 \\
\hline & BH-NS & 23.3 & 1.179 & 0.396 \\
\hline & ВН-BH & 52.6 & 77.19 & 1.031 \\
\hline \multirow[t]{3}{*}{ M20.A } & NS-NS & 260 & 1.094 & 0.082 \\
\hline & BH-NS & 272 & 15.85 & 0.405 \\
\hline & BH-BH & 1285 & 2765 & 0.973 \\
\hline \multirow[t]{3}{*}{ M26.A } & NS-NS & 374 & 1.604 & 0.082 \\
\hline & BH-NS & 297 & 15.78 & 0.402 \\
\hline & ВН-BH & 531 & 706.6 & 0.958 \\
\hline M25.A & NS-NS & 310 & 1.333 & 0.082 \\
\hline & BH-NS & 152 & 8.272 & 0.426 \\
\hline & ВН-ВН & 226 & 292.0 & 0.953 \\
\hline
\end{tabular}

Table 3. continued.

\begin{tabular}{llccc}
\hline \hline Model & Merger & $\begin{array}{c}\text { Rate density }^{(a)} \\
{\left[\mathrm{Gpc}^{-3} \mathrm{yr}^{-1}\right]}\end{array}$ & $\begin{array}{c}\text { Detection rate } \\
{\left[\mathrm{yr}^{-1}\right]}\end{array}$ & $z_{\text {hor }}{ }^{(c)}$ \\
\hline M23.A & NS-NS & 204 & 0.858 & 0.082 \\
& BH-NS & 45.4 & 2.280 & 0.397 \\
& BH-BH & $\mathbf{5 0 . 8}$ & 63.02 & 1.024 \\
\hline M30.A & NS-NS & 426 & 1.789 & 0.083 \\
& BH-NS & 113 & 6.451 & 0.433 \\
& BH-BH & $\mathbf{6 4 1}$ & 1023 & 1.152 \\
\hline M35.A & NS-NS & 524 & 2.237 & 0.082 \\
& BH-NS & 73.3 & 3.526 & 0.444 \\
& BH-BH & $\mathbf{1 0 9}$ & 108.2 & 1.152 \\
\hline M33.A & NS-NS & 321 & 1.329 & 0.082 \\
& BH-NS & 21.1 & 0.935 & 0.392 \\
& BH-BH & $\mathbf{1 9 . 5}$ & 19.41 & 1.149 \\
\hline M40.A & NS-NS & 434 & 1.822 & 0.082 \\
& BH-NS & 114 & 6.531 & 0.422 \\
& BH-BH & $\mathbf{6 2 7}$ & 947.3 & 1.152 \\
\hline M43.A & NS-NS & 323 & 1.346 & 0.082 \\
& BH-NS & 21.5 & 0.944 & 0.381 \\
& BH-BH & $\mathbf{1 9 . 5}$ & 20.07 & 1.151 \\
\hline M50.A & NS-NS & 408 & 1.691 & 0.082 \\
& BH-NS & 101 & 5.595 & 0.489 \\
& BH-BH & $\mathbf{1 1 1 4}$ & 2843 & 1.153 \\
\hline M60.A & NS-NS & 429 & 1.805 & 0.082 \\
& BH-NS & 108 & 5.727 & 0.363 \\
& BH-BH & $\mathbf{6 4 0}$ & 837.0 & 0.949 \\
\hline M70.A & NS-NS & 428 & 1.805 & 0.082 \\
& BH-NS & 108 & 6.107 & 0.408 \\
& BH-BH & $\mathbf{6 3 7}$ & 1017 & 1.107 \\
\hline
\end{tabular}

Notes. In bold we highlight BH-BH merger rate densities for all the models for easy comparison with LIGO/Virgo data. ${ }^{(a)}$ Local merger rate density at redshift $z=0$ (note rate increase with $z$ ). ${ }^{(b)}$ Detection rate for LIGO/Virgo mid-high range (O3 proxy). ${ }^{\left({ }^{c}\right)}$ Redshift for the most distant source detectable (best located/oriented).

with low star formation at high redshifts. This particular choice does not affect any of our conclusions for LIGO/Virgo as these instruments are not expected to probe sources at high redshifts $(z>2)$.

There are different PPSN models employed in models M10.B (strong PPSN; low maximum BH mass) and M30.B (weak PPSN; high maximum BH mass). However, this does not affect significantly the intrinsic merger rate density in a given volume that is presented in Fig. 10. This is because the number of $\mathrm{BH}-\mathrm{BH}$ binaries is about the same in each of the PPSN models. However, the differences in $\mathrm{BH}$ masses affect notably the detection rates. For example, compare the detection rate of $\mathrm{BH}-$ BH mergers in models M30.A/B and M60.A/B that differ only by PPSN input physics (see Table 3).

Figure 11 demonstrates the effects of the $\mathrm{CE}$ phase and natal kicks on the intrinsic BH-BH merger rate density. We use a sequence of four models with exactly the same input physics that differ only with regard to their various assumptions about the CE phase and natal kicks. With the optimistic approach to the $\mathrm{CE}$ and natal kicks (that allows for survival of the CE phase with HG donors and very low or zero BH natal kicks; model M30.A) the local $(z=0)$ merger rate density is relatively high: $R_{\mathrm{BHBH}}=$ $641 \mathrm{Gpc}^{-3} \mathrm{yr}^{-1}$. When we apply a more restrictive approach to the CE phase, but keep the same natal kicks as above (CE survival not allowed for HG donors; model M30.B), then we note a 


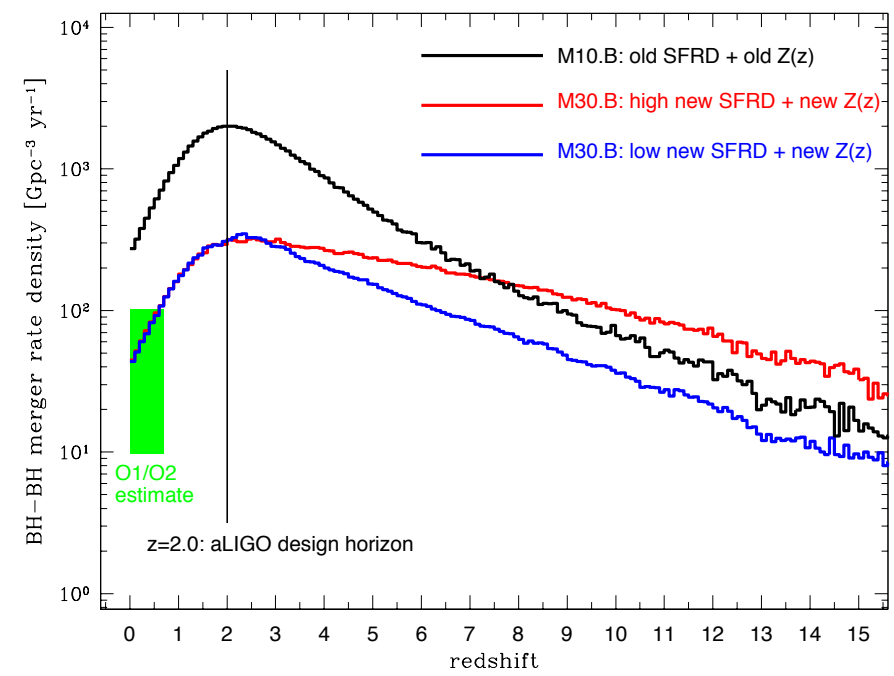

Fig. 10. Merger rate density of BH-BH mergers from Population I/II stars. We employ models M10.B and M30.B to illustrate the effects of our assumptions on the star formation rate and cosmic metallicity evolution on the BH-BH mergers. LIGO/Virgo O1/O2 constraint on the $\mathrm{BH}-\mathrm{BH}$ merger rate in local Universe is also shown. We note that the decrease of the BH-BH merger rate density at low-redshift (from old to new models) is due to the average metallicity of stars at any given redshift in old models, as it is much lower than the average metallicity of stars in new models (see Sect. 3.2 for details).

significant decrease in the rate: $R_{\mathrm{BHBH}}=43.7 \mathrm{Gpc}^{-3} \mathrm{yr}^{-1}$. Addition of the moderate natal kicks while keeping the restrictive CE-phase approach (model M35.B) decreases the rate further: $R_{\mathrm{BHBH}}=4.69 \mathrm{Gpc}^{-3} \mathrm{yr}^{-1}$. An additional increase of natal kicks (model M33.B) with the same restrictive CE-phase approach, brings the rate to a very low value: $R_{\mathrm{BHBH}}=1.37 \mathrm{Gpc}^{-3} \mathrm{yr}^{-1}$. A comparison with the LIGO/Virgo rate estimate shows that models M30.A, M35.B, M33.B are excluded. It indicates that within our limited sample of evolutionary models, some combinations of CE-phase approach and natal kicks can be excluded as not likely. For example, moderate to high natal kicks and a restrictive CE-phase treatment are an unlikely combination. Note, however, that we cannot draw conclusions about the rates based solely on the natal kicks or solely on the CE-phase approach because the results are degenerate with respect to these two major factors. For example, if we apply the optimistic CE-phase approach to models with moderate to high natal kicks then both these models fit within (or very close to) the LIGO/Virgo rate estimate. In particular, we find $R_{\mathrm{BHBH}}=109 \mathrm{Gpc}^{-3} \mathrm{yr}^{-1}$ for model M35.A (moderate natal kicks and optimistic $\mathrm{CE}=$ phase) and $R_{\mathrm{BHBH}}=19.5 \mathrm{Gpc}^{-3} \mathrm{yr}^{-1}$ for model M33.A (high natal kicks and optimistic CE-phase).

Figure 12 shows the intrinsic merger rate density for all types of mergers: NS-NS, BH-NS and BH-BH. As an example, we use model M30.A/B. As discussed above, the local BH$\mathrm{BH}$ intrinsic merger rate density for model M30. $\mathrm{B}\left(R_{\mathrm{BHBH}}=\right.$ $43.7 \mathrm{Gpc}^{-3} \mathrm{yr}^{-1}$ ) is within the LIGO/Virgo estimate. The predicted local $\mathrm{BH}-\mathrm{NS}$ intrinsic merger rate density for model M30.B $\left(R_{\mathrm{BHNS}}=11.1 \mathrm{Gpc}^{-3} \mathrm{yr}^{-1}\right)$ is within the LIGO/Virgo upper limit. The local intrinsic merger rate density for NS-NS systems is only just above LIGO/Virgo $90 \%$ level lower limit for model M30.B $\left(R_{\mathrm{NSNS}}=122 \mathrm{Gpc}^{-3} \mathrm{yr}^{-1}\right)$, while it is well within the LIGO/Virgo estimate for model M30.A $\left(R_{\mathrm{NSNS}}=\right.$ $426 \mathrm{Gpc}^{-3} \mathrm{yr}^{-1}$ ).

Detection rates (Table 3 is based on each source merger redshift, its mass (we use mass dependent waveforms for each

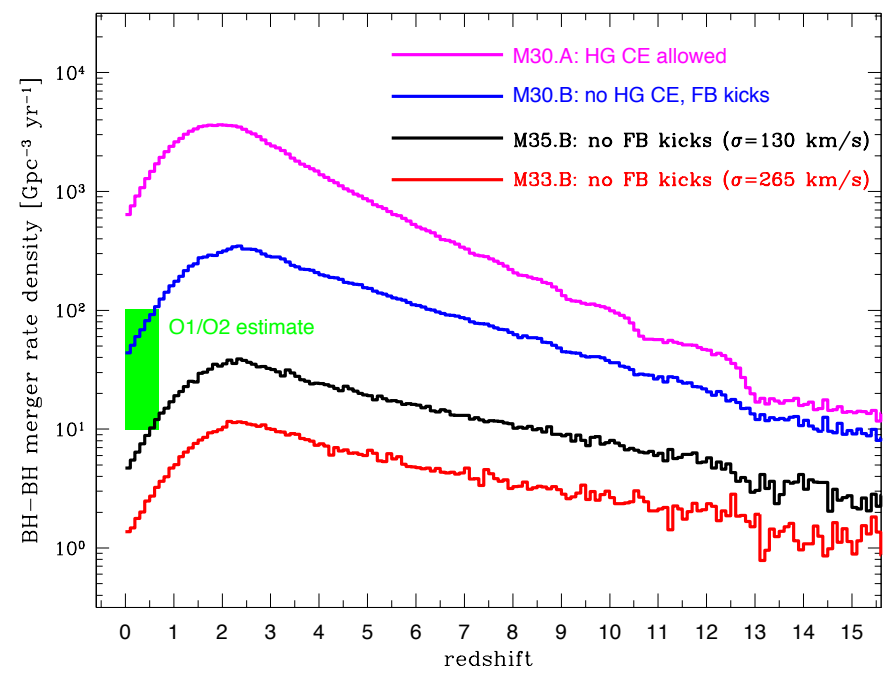

Fig. 11. Merger rate density of $\mathrm{BH}-\mathrm{BH}$ mergers for various assumptions about the common envelope and BH natal kicks. The possibility of development and survival of the CE phase with a Hertzsprung gap donor, increases the low-redshift $\mathrm{BH}-\mathrm{BH}$ merger rate density by $\sim 1$ order of magnitude: compare models M30.A and M30.B (a similar effect is found for other models). Natal kicks tend to decrease the $\mathrm{BH}-\mathrm{BH}$ merger rate density by $\sim 1.5$ order of magnitude: from fall-back attenuated natal kicks (almost no BH natal kicks: model M30.B) to full scale BH natal kicks (as high as observed for Galactic single pulsars: model M33.B). We note that the degeneracy between the tested input physics; by applying high natal kicks we can bring model M30.A down to agree with the LIGO/Virgo estimate, or by allowing for HG CE we can bring models M35.B and M33.B up to also match the LIGO/Virgo constraint.

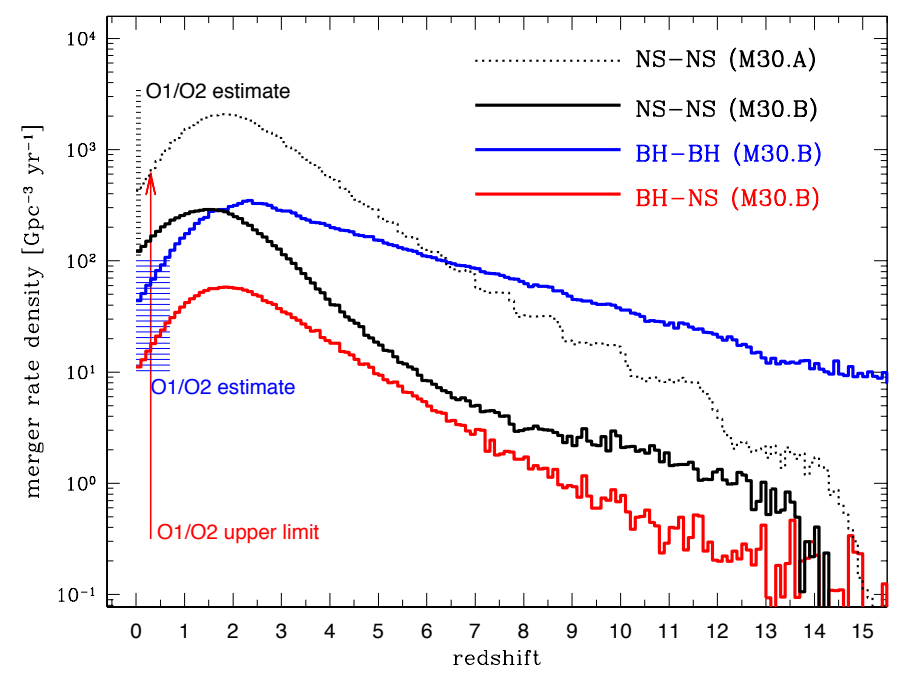

Fig. 12. Merger rate density of NS-NS, BH-NS and BH-BH mergers for model M30. For comparison, we show LIGO/Virgo O1/O2 constraints on merger rate densities. While actual estimates are available for the $\mathrm{BH}-\mathrm{BH}$ and NS-NS mergers, only an upper limit is available for the BH-NS mergers from O1/O2 data (but see Appendix A.9). We note that the presented merger rate densities are consistent with the LIGO/Virgo estimates.

merger) and take into account the LIGO detector antenna (peanut-shaped) pattern. For this particular estimate we employ LIGO sensitivity labeled "mid-high" that approximately corresponds to the current (O3) LIGO sensitivity (for details, see Appendix A.8). The detection rate is then a convolution of the merger rate density (and its change with redshift within the 


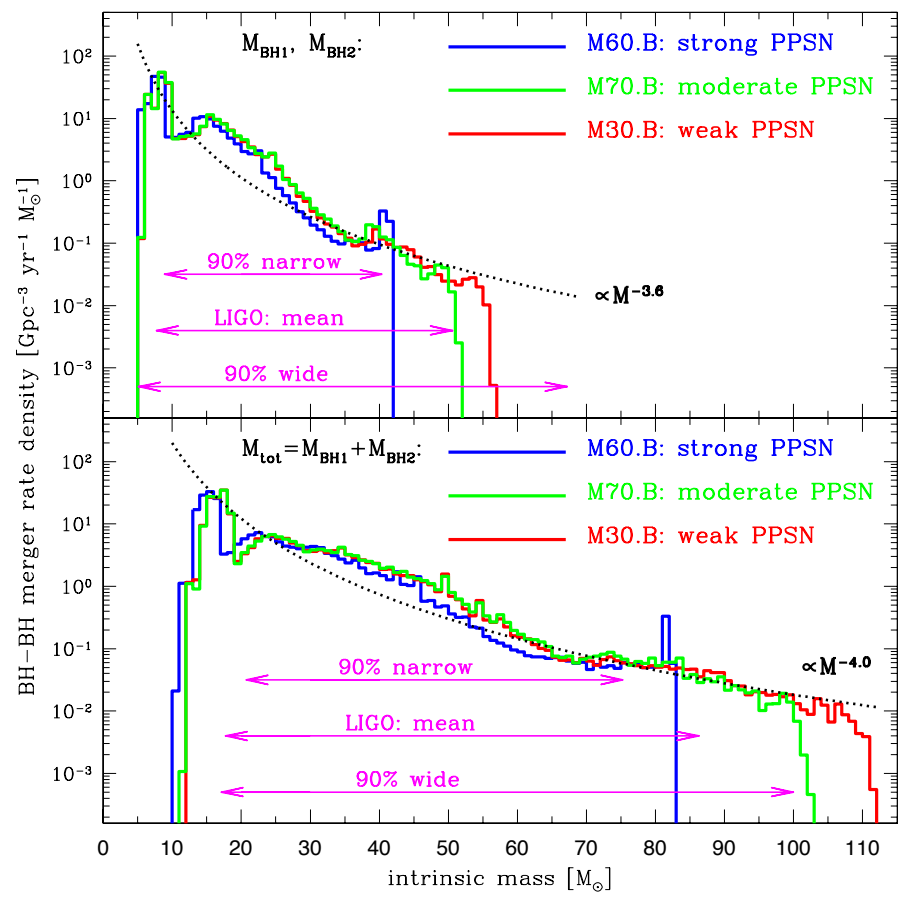

Fig. 13. BH masses in BH-BH mergers within the design advancedLIGO horizon $(z<2)$ for models M60.B (strong pair-instability pulsation supernovae), M70.B (moderate PPSN), and M30.B (weak PPSN). Top panel: distribution of individual $\mathrm{BH}$ masses, bottom panel: total $\mathrm{BH}-\mathrm{BH}$ system mass. All distributions are intrinsic (neither redshifted nor weighted by detection probability). For comparison, we also indicate the range of LIGO/Virgo O1/O2 mean mass estimates and their most narrow and the widest allowed range within $90 \%$ confidence limits. Individual BH masses may reach $\sim 40 M_{\odot}$ (M60.B) and $\sim 55 M_{\odot}(\mathrm{M} 30 . \mathrm{B})$, while the total BH-BH mass may reach $\sim 80 M_{\odot}$ (M60.B) and $\sim 110 M_{\odot}(\mathrm{M} 30 . \mathrm{B})$. Note that these distributions only very approximately resemble power-laws: $\sim M^{-3.6}$ (for individual $\mathrm{BH}$ masses) and $\sim M^{-4.0}$ (for total BH-BH mass). Power-law fits (dashed black lines) were performed for model M30.B in the log-log space.

LIGO horizon for a given source type) and the mass distribution of given source type (NS-NS, BH-NS, BH-BH). For clarity, we list the horizon redshift for the best located/oriented (directly overhead source with an orbital plane perpendicular to the line of sight) source within each merger type. This naturally corresponds to the furthest detectable redshift (distance) of the most massive source within each merger type found in our simulations.

\section{3. $\mathrm{BH}$ masses}

In this section we discuss the masses of BHs in BH-BH mergers, focusing on either the individual component masses, $M_{\mathrm{BH} 1}$ (more massive component) and $M_{\mathrm{BH} 2}$ (less massive component), or the total merger mass, $M_{\mathrm{tot}}=M_{\mathrm{BH} 1}+M_{\mathrm{BH} 2}$.

Figure 13 shows the intrinsic (source frame) distributions of the individual $\mathrm{BH}$ masses $\left(M_{\mathrm{BH} 1}\right.$ and $M_{\mathrm{BH} 2}$ shown together in one distribution) and the total $\mathrm{BH}-\mathrm{BH}$ merger masses for our three models of PPSN: M30.B (weak PPSN), M70.B (moderate PPSN), and M60.B (strong PPSN). These distributions are weighted by the intrinsic merger rate density for all $\mathrm{BH}-\mathrm{BH}$ mergers (independent of mass) within a given redshift $(z<2)$. As clearly seen, the different PPSN treatments in the models shown here impact the maximum $\mathrm{BH}$ mass generated. In our calculations, the individual $\mathrm{BH}$ masses in merging binaries extend

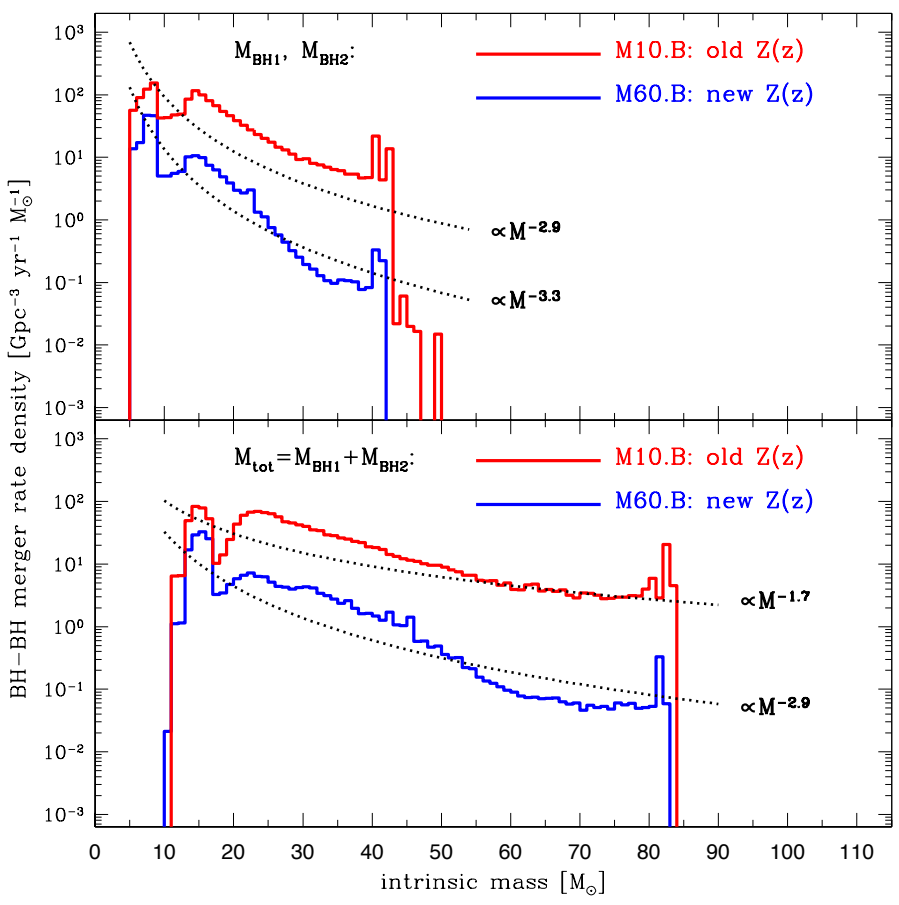

Fig. 14. $\mathrm{BH}$ masses in BH-BH mergers within design advanced-LIGO sensitivity $(z<2)$ for models M10.B (that employs old average metallicity cosmic evolution) and M60.B (new average metallicity evolution). BH masses are calculated with the same formulas in both models. Top panel: distribution of individual $\mathrm{BH}$ masses, bottom panel: total $\mathrm{BH}-\mathrm{BH}$ system mass. All distributions are intrinsic (not redshifted nor weighted by detection probability). We note that application of new metallicity evolution with redshift (less low- $Z$ stars: less high mass BHs) results in somewhat steeper BH mass distributions as contrasted with application of old metallicity evolution (more low- $\mathrm{Z}$ stars: more high mass $\mathrm{BHs}$ ). Power-law fits (dashed black lines) were performed for both models in the log-log space.

to $M_{\mathrm{BH}, \text { max }} \sim 40 M_{\odot}$ in M60.B, $M_{\mathrm{BH}, \text { max }} \sim 50 M_{\odot}$ in M70.B, and $M_{\mathrm{BH}, \max } \sim 55 M_{\odot}$ in M30.B. For comparison, among all the candidates reported by LIGO/Virgo (see Table 1), the largest mean mass for any individual $\mathrm{BH}$ is $50.6 M_{\odot}$ (for GW170729); even most optimistically (within the $90 \%$ credible limits), the largest $\mathrm{BH}$ reported in a binary is only 67.2 $M_{\odot}$ (again for GW170729). The total $\mathrm{BH}-\mathrm{BH}$ merger mass reaches $M_{\mathrm{BH}, \max } \sim 80 M_{\odot}$ in M60.B, $M_{\mathrm{BH}, \max } \sim 100 M_{\odot}$ in M70.B, and $M_{\mathrm{BH}, \max } \sim 110 M_{\odot}$ in M30.B. For comparison, LIGO/Virgo total mean BH-BH mass estimates reach $86.3 M_{\odot}$, while the $90 \%$ confidence level on the most massive $\mathrm{BH}-\mathrm{BH}$ merger is as high as $100 M_{\odot}$. The distributions approximately resemble steep power-laws: $\sim M^{-3.6}$ for individual $\mathrm{BH}$ mass, and $\sim M^{-4.0}$ for total $\mathrm{BH}-\mathrm{BH}$ mass. [Unless otherwise noted, all exponents refer to a power law fit to all merging binaries, covering the full mass range]. The power law index depends weakly on the PPSN model.

Figure 14 illustrates the effect of different $Z(z)$ assumptions on source frame $\mathrm{BH}$ mass distributions. We choose models M10.B and M60.B that utilize the same strong PPSN model, but employ slow $Z(z)$ evolution based on an older estimate and faster $Z(z)$ evolution based on a more recent estimate, respectively. For both models the range of $\mathrm{BH}$ masses (whether individual $\mathrm{BH}$ masses or total $\mathrm{BH}-\mathrm{BH}$ merger masses) is very similar. The same PPSN model leads to a similar cutoff $\mathrm{BH}$ mass at the high mass end. However, we note the change in steepness of the power-laws which approximate these distributions. For individual $\mathrm{BH}$ masses the slope changes from $\sim M^{-2.9}$ 


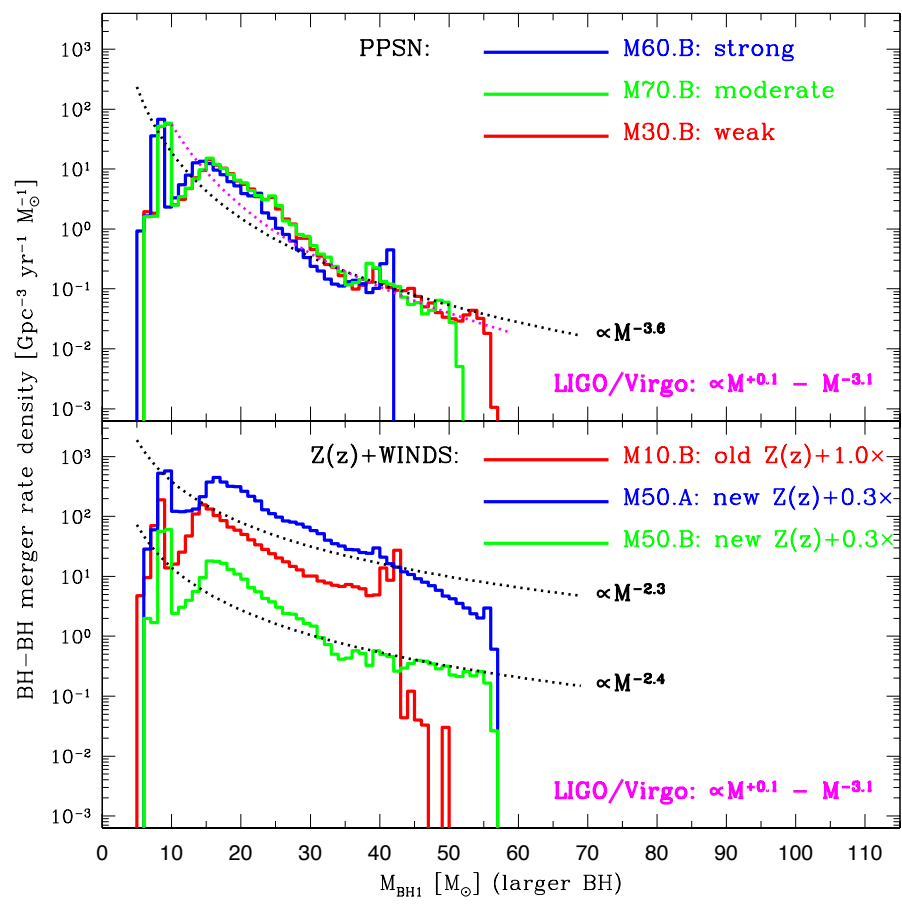

Fig. 15. Larger (more massive) BH mass distributions in BH-BH mergers within design advanced-LIGO sensitivity $(z<2)$ for models M60.B, M70.B, M30.B, M10.B, M50.A, M50.B. Power-law fits (dashed black lines) were performed for model M30.B in the top panel $\left(\propto M^{-3.6}\right)$, and for models M50.A $\left(\propto M^{-2.3}\right)$ and M50.B $\left(\propto M^{-2.4}\right)$ in the bottom panel in the log-log space. These distributions can be compared with other theoretical predictions (e.g., Fig. 1 of Mapelli et al. 2019, or Fig. 4 of Stevenson et al. 2019 that seem to be close to $\propto M^{-2}-M^{-3}$; we note that power-laws shown in the latter work are not correct) or with the LIGO/Virgo observational estimate of $\propto M^{-1.6}$ with large uncertainty on the power-law index: $\propto M^{+0.1}-M^{-3.1}$ (Fig. 1 in Abbott et al. 2019a). Some of our models (e.g., top panel) show somewhat steeper relations, while other models (bottom panel) are in agreement with LIGO/Virgo estimate.

for model M10.B to $\sim M^{-3.3}$ for model M60.B. For total BHBH masses the slope changes from $\sim M^{-1.7}$ for model M10.B to $\sim M^{-2.9}$ for model M60.B. This comes from the fact that in models with high numbers of low metallicity stars (e.g., M10.B) BH masses are (on average) higher and therefore the distributions are flatter than in models with small number of low metallicity stars (e.g., M60.B).

Figure 15 shows the intrinsic (source frame) distributions of the more massive $\mathrm{BH}$ mass $\left(M_{\mathrm{BH} 1}\right)$ weighted by the intrinsic merger rate density for all $\mathrm{BH}-\mathrm{BH}$ mergers (independent of mass) within a given redshift $(z<2)$. Here we present two groups of models. In one, we alter the amount of PPSN massloss: M60.B (strong mass-loss), M70.B (moderate mass-loss) and M30.B (weak mass-loss). All these models tend to have similar and rather steep power-law distribution of larger BH mass $\left(\propto M^{-3.6}\right)$. In the second group we show models that tend to produce shallower power-law distributions $\left(\propto M^{-2.4}\right)$ : M10.B (old $Z(z)$ relation with standard stellar winds and restrictive $C E$ treatment), M50.A (new $Z(z)$ relation with $30 \%$ reduced stellar winds and optimistic CE), and M50.B (new $Z(z)$ relation with $30 \%$ reduced stellar winds and restrictive $\mathrm{CE}$ ). Clearly models that tend to produce more low metallicity stars (e.g., M10) and models with reduced stellar winds (e.g., M50) generate more massive BHs and produce shallower distributions. However, our mass distributions are not perfect power laws, particularly at low mass.

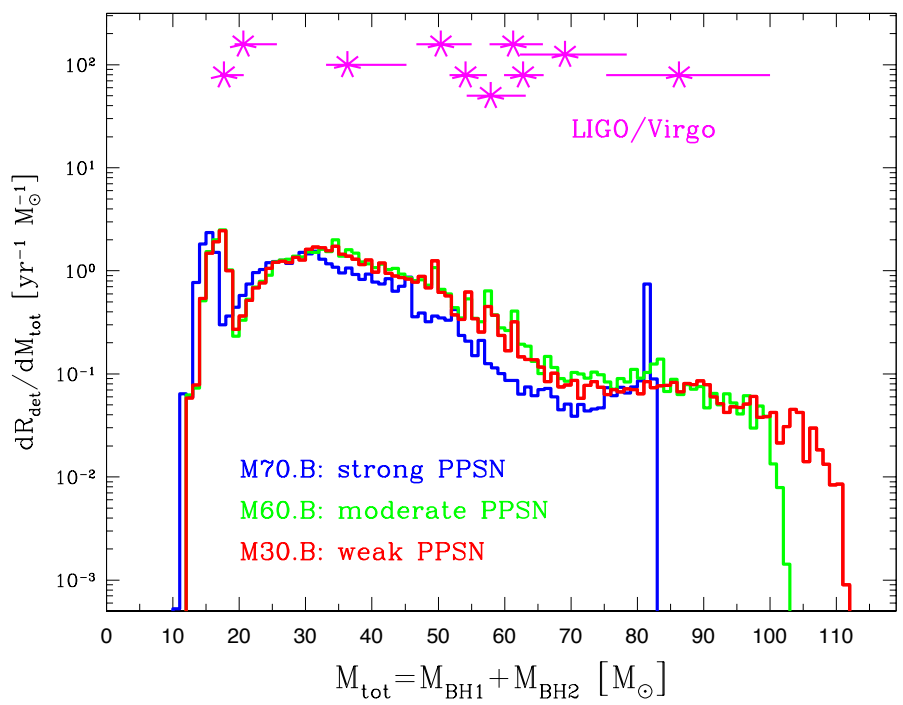

Fig. 16. Detection-weighted BH-BH merger total intrinsic (not redshifted) mass for models with different assumptions on the pairinstability pulsation supernovae: M70.B (strong PPSN), M60.B (moderate PPSN), M30.B (weak PPSN). For comparison, we also show LIGO/Virgo O1/O2 mean total mass estimates and their $90 \%$ confidence limits.

For example, a power law approximation to our simulation data restricted to the mass range of reported LIGO/Virgo observations will recover a steeper power law exponent. For comparison, LIGO/Virgo infers a phenomenological pure power law distribution of $\propto M^{+0.1}-M^{-3.1}$ (Abbott et al. 2019a based on observations spanning $M_{\mathrm{BH} 1}$; see their Fig. 1). When comparing our calculations to LIGO/Virgo observations in this way, the estimated power law exponent depends sensitively on the choice of the low-mass cutoff $M_{\mathrm{BH} 1}$, where our models predict a substantial structure in the mass distribution.

Figure 16 shows the total $\mathrm{BH}-\mathrm{BH}$ merger mass distributions weighted by the LIGO detection probability (dependent on mass). We show models with different assumptions on PPSN (M30.B, M60.B, M70.B) and contrast them with LIGO/Virgo observations. These distributions are flatter than the intrinsic mass distributions as more massive mergers are detected in larger volumes (and thus in larger numbers) than lower mass mergers. Naturally, the maximum BH-BH mass cut-offs are the same as for the intrinsic distributions. W note that within the observational uncertainties, all three of these models can reproduce the range of the detected $\mathrm{BH}-\mathrm{BH}$ total masses. However, a closer inspection of the distribution of observational points shows an overabundance of points near $M_{\mathrm{tot}} \sim 50-70 M_{\odot}$ with respect to the models. At this point, we do not attempt to match this apparent peak in the observed distribution (this can wait for another published set of detections from LIGO/Virgo) but we have only tested a single potential change to the input physics (see below) that may affect the shape of the total mass distribution for massive $\mathrm{BH}-\mathrm{BH}$ mergers.

In Fig. 17, we re-plot the model M30.B (with weak PPSN and restrictive CE-phase treatment) employing the standard theoretical estimates of wind mass-loss rates (rather high wind mass-loss rates; Vink et al. 2001). Additionally, we show the distributions for models M50.A and M50.B for which we reduce all stellar wind mass loss to $30 \%$ of the values used in all other models, but the rest of the input physics is exactly the same as in model M30.A/B. We note the appearance of a very prominent peak in the total BH-BH mass distribution at $M_{\text {tot }} \sim 80-100 M_{\odot}$ 


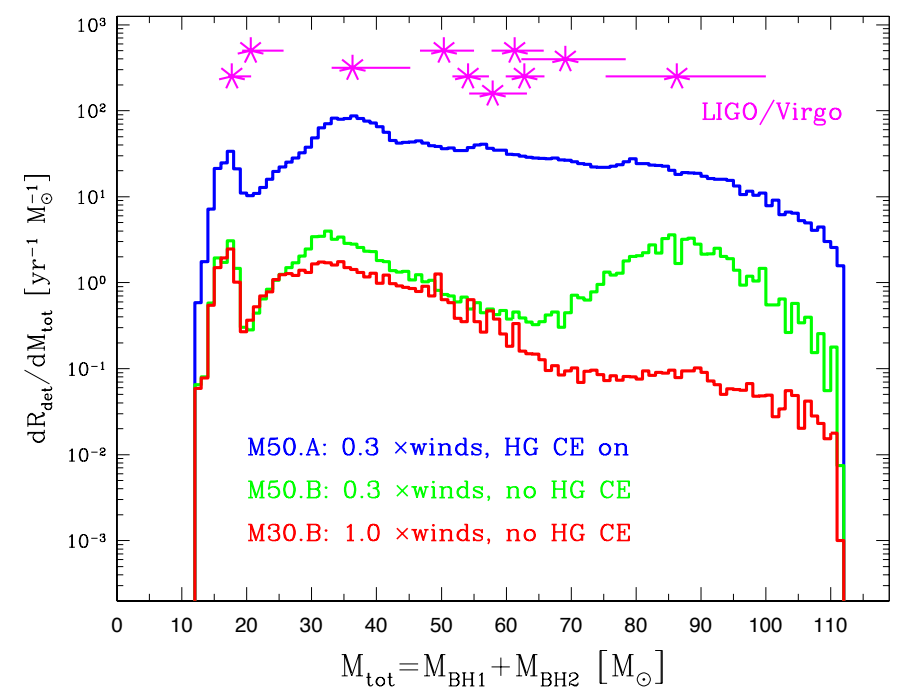

Fig. 17. Detection-weighted BH-BH merger total intrinsic (not redshifted) mass for models with different assumptions on wind mass loss and common envelope development: M50.B (weak stellar winds and optimistic CE), M50.B (weak stellar winds and pessimistic CE), M30.B (strong stellar winds and pessimistic CE). For comparison, we also show LIGO/Virgo O1/O2 mean total mass estimates and their $90 \%$ confidence limits. We note that although no model seems to reproduce the observed LIGO/Virgo BH-BH total mass distribution, we can expect that some interplay of several parameters (winds, CE, PPSN, Z(z)) may, in the future, possibly reproduce the shape of the observed distribution. For example, stronger PPSN and higher $Z(z)$ (not shown here) will tend to shift the highest BH masses to lower values. This calls for further parameter study calculations.

in model M50.B (weak PPSN and restrictive CE-phase treatment) as compared to the model M30.B distribution. That peak disappears in model M50.A (weak PPSN and optimistic CEphase treatment), but the distribution becomes much flatter than for model M30.B. These models seem to better reproduce the distribution of observational points. In Sect. 4.4 we present a brief discussion comparing synthetic StarTrack data with LIGO/Virgo data on BH masses.

\section{4. $B H-B H$ effective spins}

In this section, we present our predictions for the effective spins of BH-BH mergers. Having shown that we can reproduce the effective spins (whether low or high) with some of our adopted BH spin models (Geneva or MESA), we wish to see whether we can also reproduce the effective spin distribution (or, rather, the range of observed values) of all of the reported LIGO/Virgo BH-BH mergers.

In Fig. 18, we show the measurements of the effective spins in the LIGO/Virgo BH-BH merger observations, superimposed with the $\chi_{\text {eff }}$ predictions calculated using the $\mathrm{BH}$ natal spins from the Geneva evolutionary calculations. All of the LIGO/Virgo observations cluster around zero $\left(\chi_{\mathrm{eff}} \sim 0\right)$. For the comparison, we use two models: model M20.B, with fallback-decreased $\mathrm{BH}$ natal kicks (effectively no kicks for massive BHs, and small kicks for low mass BHs); and the model M23.B, with high BH kicks that are independent of the $\mathrm{BH}$ mass (with 1-dimensional $\sigma=265 \mathrm{~km} \mathrm{~s}^{-1}$ ). Our model distributions peak at high values $\left(\chi_{\mathrm{eff}} \sim 0.9\right)$ and then quickly fall off toward small values (see the logarithmic scale on Fig. 18).

Mergers with high values of the effective spin host BHs with high spins. In the Geneva model, these BHs can form with either

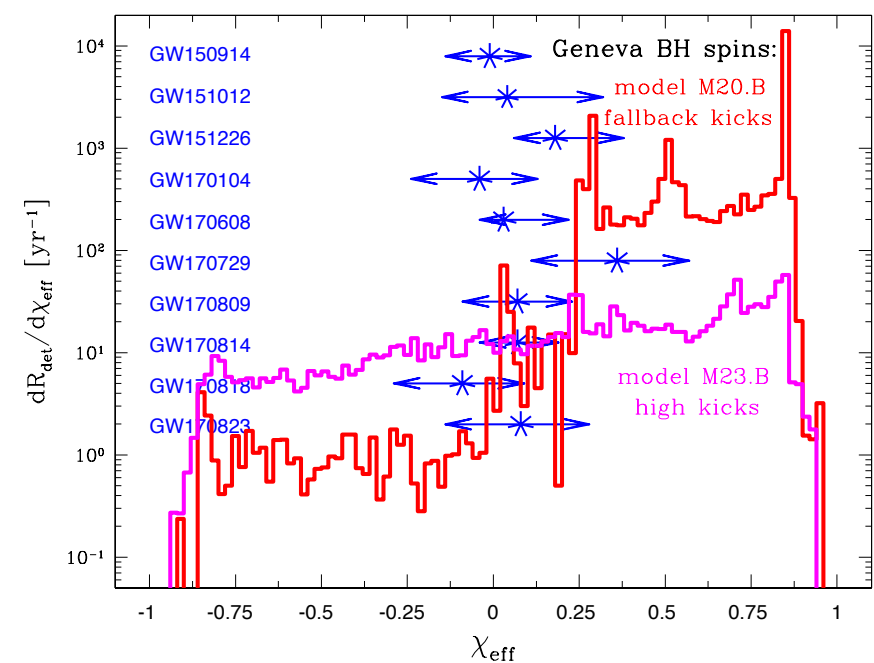

Fig. 18. Detection-weighted distribution of effective spin parameter of BH-BH mergers for model M20.B (fallback decreased BH kicks; no $\mathrm{BH}$ kicks for massive $\mathrm{BHs}$ ) and M23.B (high $\mathrm{BH}$ natal kicks with 1D $\sigma=265 \mathrm{~km} \mathrm{~s}^{-1}$ for all BHs) with Geneva mildly efficient angular momentum transport. We note that both distributions peak at high values $\left(\chi_{\text {eff }} \sim 0.9\right)$. Natal kicks decrease the effective spin parameter (average $\chi_{\text {eff }}=0.3$; M23.B) as compared to model with almost no BH kicks (average $\chi_{\mathrm{eff}}=0.7 ;$ M20.B). For comparison, we show the $90 \%$ credible limits (blue arrows) and the most likely values (blue stars) of the effective spin parameter for ten LIGO/Virgo BH-BH mergers. Although we can recover all the reported values, the predicted peak of $\chi_{\text {eff }}$ distribution is not coincident with the current LIGO/Virgo data. It indicates that BHs have typically lower spins than resulting from the Geneva model for $\mathrm{BH}$ natal spins, or that the detected $\mathrm{BH}-\mathrm{BH}$ mergers are not formed in the classical isolated binary evolution.

low or high masses, depending on the chemical composition of the progenitor stars (Fig. 1). Mergers with low effective spins originate predominantly from systems with high-mass BHs. Strong natal kicks (as in model M23.B) produce higher misalignment of the BH spins with respect to the binary's angular momentum vector during the $\mathrm{BH}$ formation, and this decreases the value of the effective spin. We note that although both distributions are similar and peak at high values, natal kicks decrease the effective spin parameter (average $\chi_{\text {eff }}=0.3 ;$ M23.B) as compared to model with almost no BH kicks ( $\chi_{\text {eff }}=0.7$; M20.B). The fraction of $\mathrm{BH}-\mathrm{BH}$ mergers with negative $\chi_{\mathrm{eff}}$ is sizeable in model M23.B (27.4\%), while it is negligible in model M20.B $(\sim 0.3 \%)$.

We can recover all the reported values within both models (more easily with model M23.B than with M20.B), but our predicted $\chi_{\text {eff }}$ distributions using Geneva spins as inputs are not consistent with the current LIGO/Virgo data. For example, for each of our models and for $N$ observations, we can ask whether we would expect at least one measurement with a maximum value of $\chi_{\text {eff }}$ above what has been observed thus far; that is, $1-P\left(<\chi_{\text {eff }}\right)^{N}$, where $P\left(<\chi_{\mathrm{eff}}\right)$ is the cumulative distribution implied by the detection-weighted $\chi_{\mathrm{eff}}$ distribution reported in Fig. 18. Even considering only subsets of events, such as the high-mass or low-mass events, we find a small $\left(1.1 \times 10^{-4}\right.$ for M23.B $)$ to infinitesimal $\left(6.7 \times 10^{-10}\right.$ for M20.B) probability that the predicted spin distribution is compatible with observations reported to date.

We note that to reach this conclusion, we have used two extreme natal kick models. In particular, we tested a model with high $\mathrm{BH}$ natal kicks that tends to maximize the $\mathrm{BH}$ 


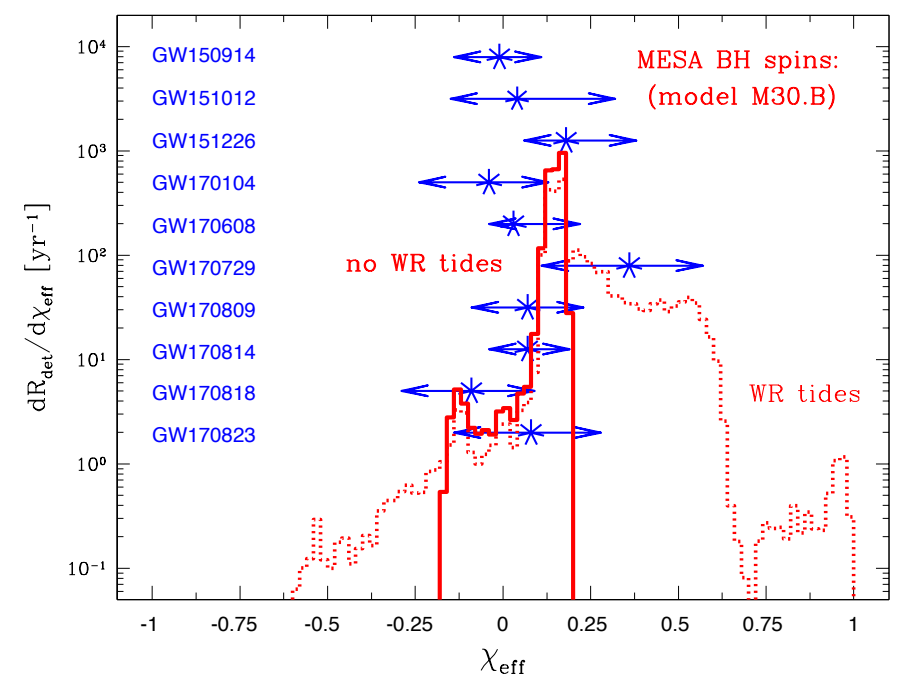

Fig. 19. Detection-weighted distribution of the effective spin parameter of BH-BH mergers for model M30.B with MESA efficient angular momentum transport and fallback decreased BH kicks (no BH kicks for massive BHs). We either do not allow for efficient tidal spin-up of WR stars that are the most common immediate progenitors of $\mathrm{BHs}$ in our models (natal BH spin is calculated directly from MESA stellar models) or we take it into account (natal BH spin is then calculated as described in Sect. 2.5 if the WR star progenitor was subject to an efficient tidal spin-up). For the "no WR tides" approach we find a rather narrow distribution of effective spins $\left(-0.2 \lesssim \chi_{\text {eff }} \lesssim 0.2\right)$ that is peaked at positive values (average $\chi_{\text {eff }}=0.15$ ). For efficient "WR tides" the distribution is broad $\left(-0.5 \lesssim \chi_{\text {eff }} \lesssim 1.0\right)$ with a peak at $\chi_{\text {eff }} \sim 0.15(\sim 73 \%)$ and a tail with $\chi_{\text {eff }} \gtrsim 0.25(27 \%)$. For comparison we show the $90 \%$ credible limits (blue arrows) and the most likely values (blue stars) of the effective spin parameter for ten LIGO/Virgo BH-BH mergers.

spin-orbit misalignment and therefore decrease our predicted effective spins. Although $\mathrm{BH}$ natal kicks as high as those adopted in model M23.B (with average 3-dimensional velocity of $\sim 400 \mathrm{~km} \mathrm{~s}^{-1}$ ) cannot yet be observationally excluded (Belczynski et al. 2016b), it is unlikely for BHs to receive larger natal kicks than the ones that have been adopted in this model (Mandel 2016; Repetto et al. 2017). We note that in these models, we allow neither for any processes that could realign the $\mathrm{BH}$ spins, nor for an effective tidal spin-up of stars in the binary progenitors of $\mathrm{BH}-\mathrm{BH}$ mergers. These processes might increase the effective spin parameter for some $\mathrm{BH}-\mathrm{BH}$ progenitors, thus shifting our results for Geneva model of $\mathrm{BH}$ natal spins even further away from the LIGO/Virgo observations.

In Fig. 19, we present the effective spin distribution for the model M30.B that employs the MESA BH natal spins. We show two versions of this model: one in which we do not allow and one in which we do allow for efficient tidal interactions in close binaries (that host WR stars; see Sect. 2.5). The version with no efficient tides shows a narrow distribution of effective spins: $-0.2 \lesssim \chi_{\text {eff }} \lesssim 0.2$ that is peaked at positive values: average $\left(\chi_{\text {eff }}=0.15\right)$. This limited range of effective spins follows directly from the underlying $\mathrm{BH}$ natal spin model that allows only for narrow range of BH spin magnitudes $a_{\text {spin }} \sim 0.05-0.15$ (see Fig. 2). The BH spins are, at most, moderately increased by accretion from the binary companion (see Figs. 8 and 9) and this tends to slightly extend the distribution to higher positive values. The distribution is only moderately affected by small-to-no BH natal kicks (fall-back decreased kicks are employed in this model) producing a small population of $\mathrm{BH}-\mathrm{BH}$ mergers with negative effective spins. On the other hand the variation with the

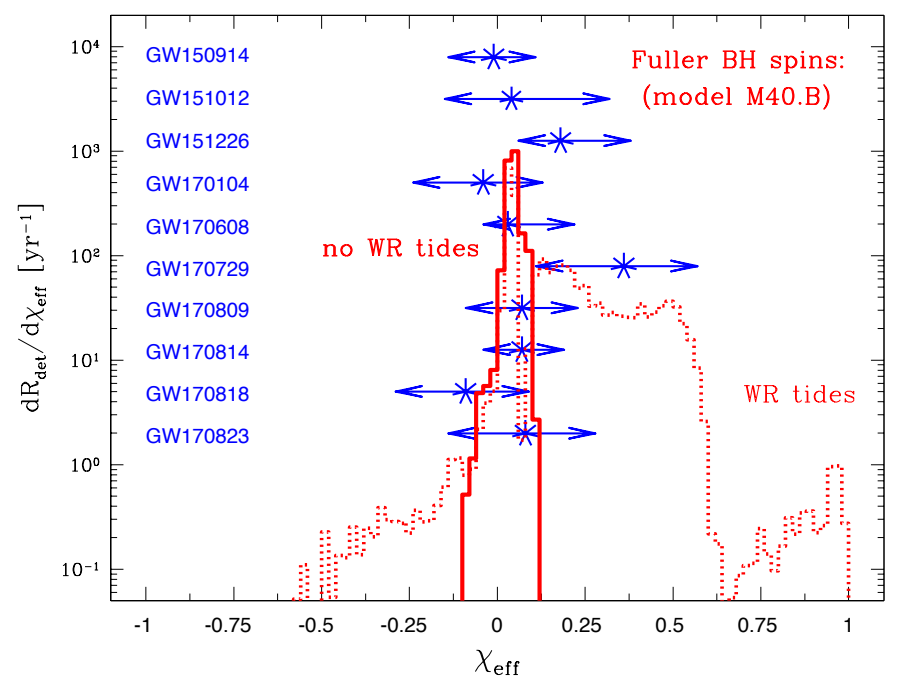

Fig. 20. Detection-weighted distribution of the effective spin parameter of BH-BH mergers for model M40.B with Fuller super-efficient angular momentum transport and fallback decreased BH kicks (no BH kicks for massive BHs). We either do not allow for the efficient tidal spin-up of WR stars that are the most common immediate progenitors of BHs in our models (natal BH spin is calculated directly from MESA stellar models) or we take it into account (natal $\mathrm{BH}$ spin is then calculated as described in Sect. 2.5 if the WR star progenitor was subject to an efficient tidal spin-up). For the "no WR tides" approach we find very narrow distribution of effective spins $\left(-0.1 \lesssim \chi_{\text {eff }} \lesssim 0.1\right)$ that is peaked at positive values (average $\chi_{\text {eff }}=0.05$ ). For efficient "WR tides" the distribution is broad $\left(-0.5 \lesssim \chi_{\text {eff }} \lesssim 1.0\right)$ with a peak at $\chi_{\text {eff }} \sim 0.05$ $(\sim 78 \%)$ and a tail with $\chi_{\text {eff }} \gtrsim 0.25(22 \%)$. For comparison, we show $90 \%$ credible limits (blue arrows) and the most likely values (blue stars) of effective spin parameter for ten LIGO/Virgo BH-BH mergers.

efficient WR tides generates a rather broad effective spin distribution: $-0.5 \lesssim \chi_{\text {eff }} \lesssim 1.0$ with a peak at: $\chi_{\text {eff }} \sim 0.15(\sim 80 \%)$ and a tail with $\chi_{\text {eff }} \gtrsim 0.25(27 \%)$. This is because in this variation about $1 / 3$ of systems are subject to significant tidal spin-up of at least one binary component and they produce large $\mathrm{BH}$ spins. The tail shows a significant drop beyond $\chi_{\text {eff }} \gtrsim 0.6$. Systems with $0.25 \lesssim \chi_{\text {eff }} \lesssim 0.6$ are these in which only one binary component was subject to tidal spin-up, while systems with $\chi_{\text {eff }} \gtrsim 0.6$ are those with both binary components being subject to tidal spinup in close WR-WR binaries.

In Fig. 20, we present the effective spin distribution for model M40.B that employs Fuller BH natal spins. We also show two versions of this model: one without efficient WR tides and one with the tides. The distribution is very narrow: $-0.1 \lesssim \chi$ eff $\lesssim$ 0.1 and is peaked at positive values: average $\chi_{\text {eff }}=0.05$. This comes directly from the assumption of very low natal $\mathrm{BH}$ spins: $a_{\text {spin }}=0.01$ (see Eq. (5)). For such a low value of natal $\mathrm{BH}$ spins, the effective spin of $\mathrm{BH}-\mathrm{BH}$ mergers is $\chi_{\text {eff }} \sim 0$. Binary accretion onto the second-born $\mathrm{BH}$ spreads effective spins to $\chi_{\text {eff }} \sim 0.1$, while small natal kicks applied to some (low-mass) $\mathrm{BHs}$ in this model create a tail extending to low negative values $\chi_{\text {eff }} \sim-0.1$. For efficient "WR tides" the distribution is broad: $-0.5 \lesssim \chi_{\text {eff }} \lesssim 1.0$ with a peak at $\chi_{\text {eff }} \sim 0.05(\sim 78 \%)$ and a tail with $\chi_{\text {eff }} \gtrsim 0.25(22 \%)$.

In Figs. 21 and 22, we present the effect of high $\mathrm{BH}$ natal kicks on the effective spin distributions in models that employ MESA and Fuller BH natal spins. For MESA BH natal kicks we use two models: M30.B (fallback-decreased natal kicks: lowto-no BH kicks), and M33.B (high natal kicks, independent of $\mathrm{BH}$ mass, drawn from a 1D Maxwellian distribution with 


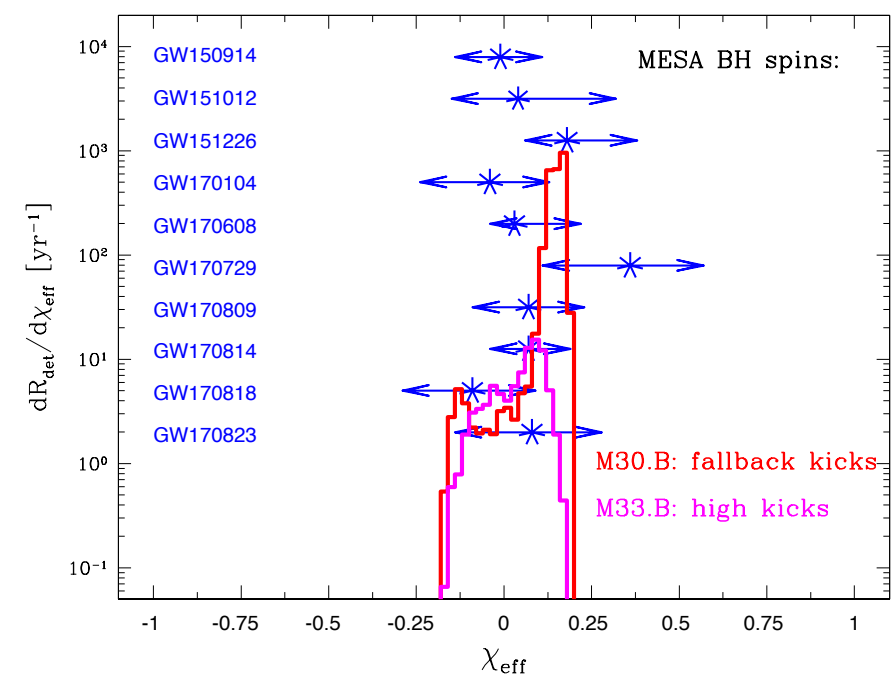

Fig. 21. Detection-weighted distribution of the effective spin parameter of BH-BH mergers for model M30.B with fallback decreased natal kicks (low natal BH kicks for low-mass BHs and no natal kicks for highmass BHs) and for model M33.B with high natal kicks (all BHs, independent of mass, are subject to natal kicks drawn from a 1D Maxwellian distribution with $\sigma=265 \mathrm{~km} \mathrm{~s}^{-1}$ ). We note that the average effective spin decreases from model M30.B $\left(\chi_{\text {eff }}=0.15\right)$ to model M33.B $\left(\chi_{\text {eff }}=0.04\right)$ as an effect of natal kicks that tend to misalign BH spins and lower the effective spin (see Eq. (1)). For comparison, we show the $90 \%$ credible limits (blue arrows) and the most likely values (blue stars) of the effective spin parameter for ten LIGO/Virgo BH-BH mergers.

$\sigma=265 \mathrm{~km} \mathrm{~s}^{-1}$ ). We note that the average effective spin decreases from model M30.B: $\chi_{\text {eff }}=0.15$ to model M33.B: $\chi_{\text {eff }}=0.04$. This is an effect of the natal kicks that tend to misalign BH spins and lower the effective spin. For Fuller BH natal kicks, we use two models: M40.B (fallback-decreased natal kicks) and M43.B (high natal kicks). We also note that the average effective spin decreases with increasing natal kicks: from model M40.B: $\chi_{\mathrm{eff}}=0.05$ to model M43.B: $\chi_{\mathrm{eff}}=0.004$.

\section{Discussion}

\subsection{Angular momentum transport in massive stars}

In this section, we discuss the dependence of the final angular momentum of the star at core collapse on angular momentum transport prescriptions and initial (ZAMS) rotation rate.

In order to evaluate the dependence of the final angular momentum on angular momentum transport prescriptions and initial rotation rate, we ran three additional $32 M_{\odot}$ models at a metallicity of $Z=0.002$ : a slow initial rotation $\left(V_{\mathrm{ini}}=\right.$ $100 \mathrm{~km} \mathrm{~s}^{-1}$ ) non-magnetic ("noTS", where TS stands for the Tayler-Spruit dynamo) model with both the Geneva and MESA code as well as a slow initial rotation $\left(V_{\text {ini }}=100 \mathrm{~km} \mathrm{~s}^{-1}\right)$ magnetic ("TS") model with the MESA code. The specific angular momentum profile of these models at the end of core He-burning are shown in Fig. 23. The helium core, which will form the bulk of the black hole, extends from the center to about $12 M_{\odot}$ in all the models plotted. For comparison, we also show the specific angular momentum profiles for our two fast initial rotation models $\left(V_{\text {ini }} / V_{\text {crit }}=40 \%: V_{\text {ini }} \approx 340 \mathrm{~km} \mathrm{~s}^{-1}\right)$ for $32 M_{\odot}$ star with $Z=0.002$ calculated with MESA-TS and Geneva no-TS assumptions. We note that these two fast models are used to calculate $\mathrm{BH}$ spin magnitudes employed in our population synthe-

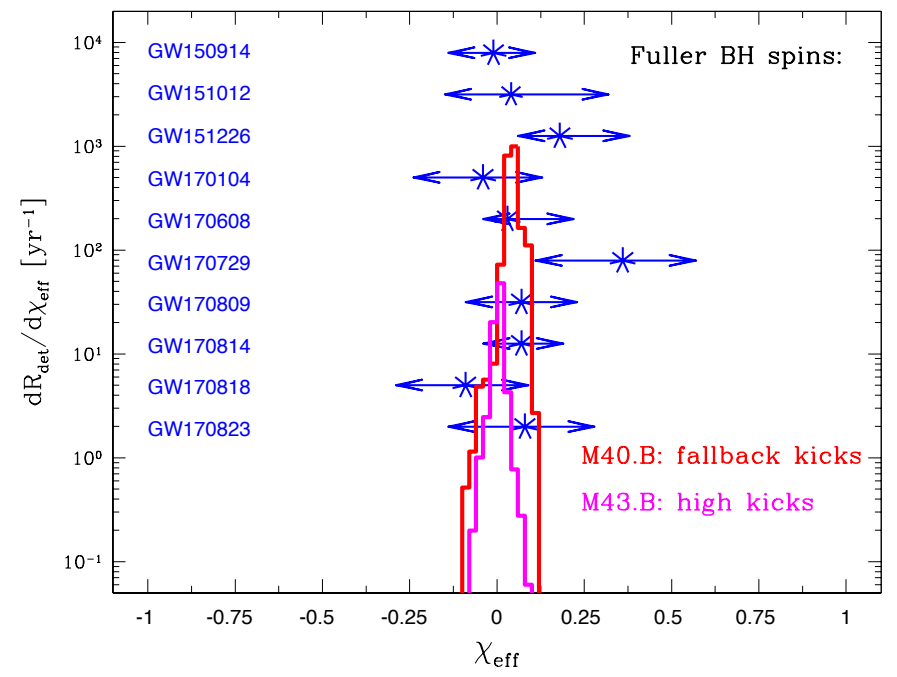

Fig. 22. Detection-weighted distribution of the effective spin parameter of BH-BH mergers for model M40.B with fallback decreased natal kicks (low natal BH kicks for low-mass BHs and no natal kicks for highmass BHs) and for model M43.B with high natal kicks (all BHs, independent of mass, are subject to natal kicks drawn from a 1D Maxwellian distribution with $\left.\sigma=265 \mathrm{~km} \mathrm{~s}^{-1}\right)$. We note that the average effective spin decreases from model M40.B $\left(\chi_{\text {eff }}=0.05\right)$ to model M43.B $\left(\chi_{\text {eff }}=0.004\right)$ as an effect of the natal kicks that tend to misalign $\mathrm{BH}$ spins and lower the effective spin (see Eq. (1)). For comparison, we show the $90 \%$ credible limits (blue arrows) and the most likely values (blue stars) of the effective spin parameter for ten LIGO/Virgo BH-BH mergers.

sis calculations (and that are referred to as MESA BH spins and Geneva BH spins).

In comparing various models, we observe the following dependencies. First, comparing the magnetic slow and fast rotation MESA models (dashed red and purple lines, respectively), we see that they end with a very similar final angular momentum $\left(a_{\text {spin }}=0.084\right.$ and $a_{\text {spin }}=0.087$ for the slow and fast rotation models, respectively ${ }^{5}$ ). There is, thus, very little dependence on the initial velocity in magnetic models. This is due to a weaker magnetic instability (and thus less efficient angular momentum transport) in slower rotation models. We would thus expect slower rotation magnetic models to have a very similar final angular momentum content as the fast models listed in Table A.2. Second, in comparing the magnetic (MESA slow and fast rotation; dashed red and purple lines) and nonmagnetic models (MESA and GENEVA no-TS slow rotation and GENEVA fast rotation no-TS models; blue, green and orange lines), we see that the non-magnetic models have final angular momenta that are more than a factor of 10 higher than the magnetic models (the angular momentum profile of the MESA fast rotation TS model multiplied by a factor of 10 is shown to facilitate the comparison; brown line). This means that for the "lower" end of the massive star range, non-magnetic models predict BHs rotating near or above critical rotation $\left(a_{\text {spin }} \gtrsim 0.9\right)$. Third, comparing the slow rotation, non-magnetic Geneva and MESA models (solid orange and green lines, respectively), the final angular momentum is very similar. The small differences in angular momentum profiles arise from small differences of the core sizes between the two codes (a result of the different treatment of the convective boundary mixing). We thus see

5 The values of dimensionless spun parameter, correspond to a specific angular momentum of Fig. $23 \sim 4.54 \times 10^{15} \mathrm{~cm}^{2} \mathrm{~s}^{-1}$; see Eq. (2). 


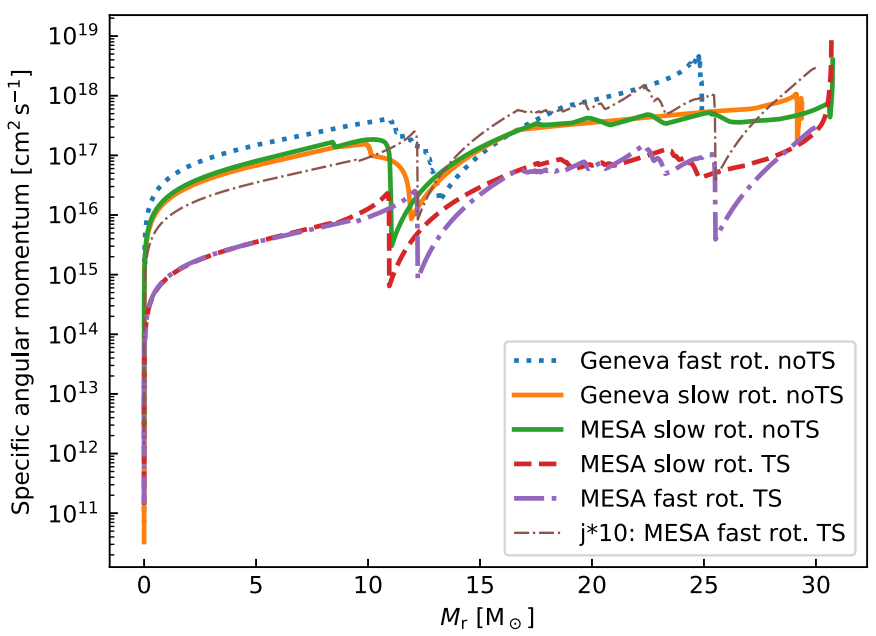

Fig. 23. Specific angular momentum profile at the end of core Heburning for the $32 M_{\odot}$ models at $Z=0.002$ calculated with a range of physical ingredients and initial rotation rates: the Geneva fast rotation model (model listed in Table A.1 with $V_{\text {ini }} / V_{\text {crit }}=40 \%, V_{\text {ini }}=$ $341.9 \mathrm{~km} \mathrm{~s}^{-1}$, dotted blue line), the Geneva slow rotation model $\left(V_{\text {ini }}=\right.$ $100 \mathrm{~km} \mathrm{~s}^{-1}$, solid orange line). Both these Geneva models do not include the Tayler-Spruit magnetic dynamo ("noTS"). We also show: the MESA slow rotation non-magnetic ("noTS") model $\left(V_{\text {ini }}=100 \mathrm{~km} \mathrm{~s}^{-1}\right.$, solid green line), the MESA slow rotation magnetic ("TS") model ( $V_{\mathrm{ini}}=$ $100 \mathrm{~km} \mathrm{~s}^{-1}$, dashed red line), and the MESA fast rotation magnetic ("TS") model (model listed in Table A.2, dot-dashed purple line). The brown thin dot-dashed line corresponds to the specific angular momentum of the MESA fast rotation model multiplied by a factor of 10 to facilitate comparisons.

that the two codes give consistent results when using a similar treatment of the angular momentum transport (without magnetic fields).

The angular momentum correlations listed above apply directly to black hole spins. Thus, we expect slow- and fastrotating magnetic models to end up in low BH spins $\left(a_{\text {spin }} \sim 0.1\right)$ and non-magnetic slow- and fast-rotating models to result in BHs with large spins $\left(a_{\text {spin }} \gtrsim 0.8\right)$. The change of the initial stellar rotation from fast $\left(\sim 340 \mathrm{~km} \mathrm{~s}^{-1} ; 40 \%\right.$ critical $)$ to slow $\left(\sim 100 \mathrm{~km} \mathrm{~s}^{-1}\right)$ does not significantly affect our estimates of the $\mathrm{BH}$ natal spin. In Appendix A.2, we present more detailed information and show that even when we increase rotation to $\sim 680 \mathrm{~km} \mathrm{~s}^{-1}$ (80\% critical) our conclusions remain unchanged (see also Fig. A.1). We note that the observed distribution of massive star spins is rather broad $\left(0-600 \mathrm{~km} \mathrm{~s}^{-1}\right)$, with two distinctive peaks: one (dominant) at $\sim 100 \mathrm{~km} \mathrm{~s}^{-1}$ and one (small) at $\sim 400 \mathrm{~km} \mathrm{~s}^{-1}$ (Ramírez-Agudelo et al. 2013). These correlations were studied for a single mass $\left(32 M_{\odot}\right.$ : the lower end of the massive star range) at a single metallicity $(Z=0.002)$ and with two models of angular momentum transport (the Tayler-Spruit theory for the magnetic dynamo and meridional currents which dominate angular momentum transport while TS dynamo is switched off). More in-depth studies, especially for higher masses, are needed to confirm these correlations.

Our models also make predictions for the birth spin periods of neutron stars and it is worthwhile comparing these predictions with the estimates of these spin periods from observations. Stars with zero-age main sequence masses lying between $\sim 8-20 M_{\odot}$ (Fryer et al. 1999, 2012) and more massive stars whose mass loss is sufficiently extreme to dramatically alter the $\mathrm{CO}$ core mass collapse to form neutron stars (through normal supernovae). If the $\mathrm{CO}$ cores are rotating and the magnetic fields are sufficiently strong, these neutron stars will emit as pulsars. By studying the distribution of spins in these pulsars, we can place constraints on the rotation period of the stars prior to collapse. This spin distribution also could provide some indication of the role angular momentum can play in normal "core-collapse" supernovae. However, in Appendix A.6 we study this problem and arrive to the conclusion that NS spins cannot be directly used to test angular momentum transport in massive stars as various (highly uncertain) mechanisms may spin down or spin up NS after its formation.

\subsection{Using single stellar models in binary simulations}

Although we use single stellar models to estimate the $\mathrm{BH}$ natal spins (see Sect. 2.1), we use binary evolution models to estimate the BH-BH merger effective spins (see Sect. 2.6). This is not fully consistent as our approach does not take into account an important effect in binary evolution that may influence the natal $\mathrm{BH}$ spin, that is, the spin-up of stars (progenitors of $\mathrm{BHs}$ ) during the RLOF phases. This process typically affects the secondary star of the BH-BH progenitors (Figs. 8 and 9; see the second evolutionary stage in both cases). Depending on the donor mass and our assumption on how much mass is accreted during the RLOF (in most models, we allow for $50 \%$ of mass lost by the donor to be accreted by its companion; but see models M20.A/B-M26.A/B) the main sequence secondary accretes anywhere from several to several tens of solar masses. This is most likely enough to significantly spin-up even a massive star (Packet 1981). However, there is a good chance that at least some of this extra angular momentum is later removed from the secondary star.

When the accretion and spin-up end, the secondary star is still on the main sequence. It will be therefore subject to angular momentum transport and losses (through winds) during a significant fraction of its subsequent lifetime. Obviously, there is less time in our binary evolution sequences for secondaries to lose extra angular momentum as compared with single stellar models initiated at ZAMS. On the other hand, there are binary interactions that help the secondary stars to get rid of extra angular momentum after the RLOF/accretion phase.

This happens when the secondary star expands significantly after the RLOF and leaving the main sequence and is subject to tidal spin-down as this occurs on a very wide orbit (Figs. 8 and 9: see the third and fourth evolutionary stage in both cases). Then the expansion leads to a CE phase, and the entire secondary's H-rich envelope (with all its angular momentum content) is removed from the binary (Figs. 8 and 9: see the fifth evolutionary stage in both cases). Any extra-angular momentum from accretion during the main sequence that is left in the Hecore of the secondary can contribute to an extra spin of the $\mathrm{BH}$ with respect to single stellar models but this would not change our conclusions significantly. If we increased the spin magnitudes of the secondary $\mathrm{BHs}$, then the distributions of $\mathrm{BH}-\mathrm{BH}$ effective spin parameters would become somewhat wider and the effective spins would mostly shift to larger positive values. This would be qualitatively similar to the effect of efficient WR tides on secondary stars (see Figs. 19 and 20) for models with the effective angular momentum transport (MESA and Fuller models). For the model with inefficient angular momentum transport (Geneva model), the BH spins (on average) are already high so increasing stellar spin may only shift this model (in terms of 
effective spin parameter) even further away from LIGO/Virgo observations.

Additionally, scenario, in which the star is stripped of its envelope, does not generally affect the trend in rotation rates discussed in Sect. 4.1. Indeed, the effective angular momentum transport through a magnetic dynamo only operates when there is a shear allowing the development of a magnetohydrodynamic instability. Hence, different rotation rates between the core and the envelope are crucial since they create the shear. In models, including magnetic fields, the transport of most of the angular momentum occurs during the short phase between core hydrogen depletion and core helium ignition (during this short phase, the core contracts and spins up whereas the envelope expands and slows down, thus creating a strong shear). Therefore, any stripping through binary interaction during core helium burning or afterwards does not affect significantly the slowdown of the cores. Stripping during the MS, however, is more complicated. There are two opposite effects. The first is that mass loss removes also angular momentum. The second is that if the entire hydrogen-rich layer is removed, then there is no envelope left to expand at the end of the MS and the spin-down of the core by internal angular momentum transport (core-envelope coupling) is reduced. In non-magnetic models, spin-down of the core is weak anyway and models still end up with relatively large spin values, $a_{\text {spin }}: 0.25$ to maximum spin (see Table A.1). In magnetic models, removal of the hydrogen-rich outer layer significantly reduces the spin-down of the core in the contraction phase after the MS and the core may keep its fast rotation rate (see also Fuller \& Ma 2019).

The majority of binaries that produce NS-NS/BH-NS/BH$\mathrm{BH}$ mergers in our models have large initial orbital separations ( $\gtrsim 1000 R_{\odot}$; de Mink \& Belczynski 2015). Therefore, binary interactions (CE or stable RLOF; see Figs. 8 and 9) that remove $\mathrm{H}$-rich stellar envelopes happen either at the end of $\mathrm{HG}$ or during $\mathrm{CHeB}$ so that the values of the natal compact body spin are not significantly affected.

Therefore, one may safely conclude that neither accretion spin-up of stars nor increasing the initial stellar rotation rate (see Sect. 4.1) can significantly modify our conclusions about the natal spins of merging binary compact objects resulting from isolated evolution. However,we do not include quasi-homogeneous evolution scenarios discussed by Chrimes et al. (2020).

We note that other processes that can affect $\mathrm{BH}$ spin in binary evolution are taken into account in our calculations. Besides tidal interactions that we probe with recent models presented in literature (see Sect. 2.5), we also account for accretion onto $\mathrm{BH}$ that may lead to BH spin-up. The spin-up is calculated using the formalism presented by Belczynski et al. (2008a). There are two potential phases of accretion onto the first-formed $\mathrm{BH}$ in our major evolutionary scenario: during the $\mathrm{CE}$ phase from the $\mathrm{H}$-rich envelope of the secondary and after the CE phase from the He-rich wind of the WR secondary (Figs. 8 and 9; see the fifth and sixth evolutionary stage in both cases). Since accretion leads to (usually very small or only modest) increase of the first $\mathrm{BH}$ spin magnitude, it tends to slightly broaden the effective spin parameter distribution.

\section{3. $B H-B H / B H-N S / N S-N S$ merger rate densities}

The predicted merger rate density of coalescing double compact object binaries depends on the particular formation scenario which is assumed. In the following two sections we discuss our merger rates obtained for isolated binary evolution and contrast

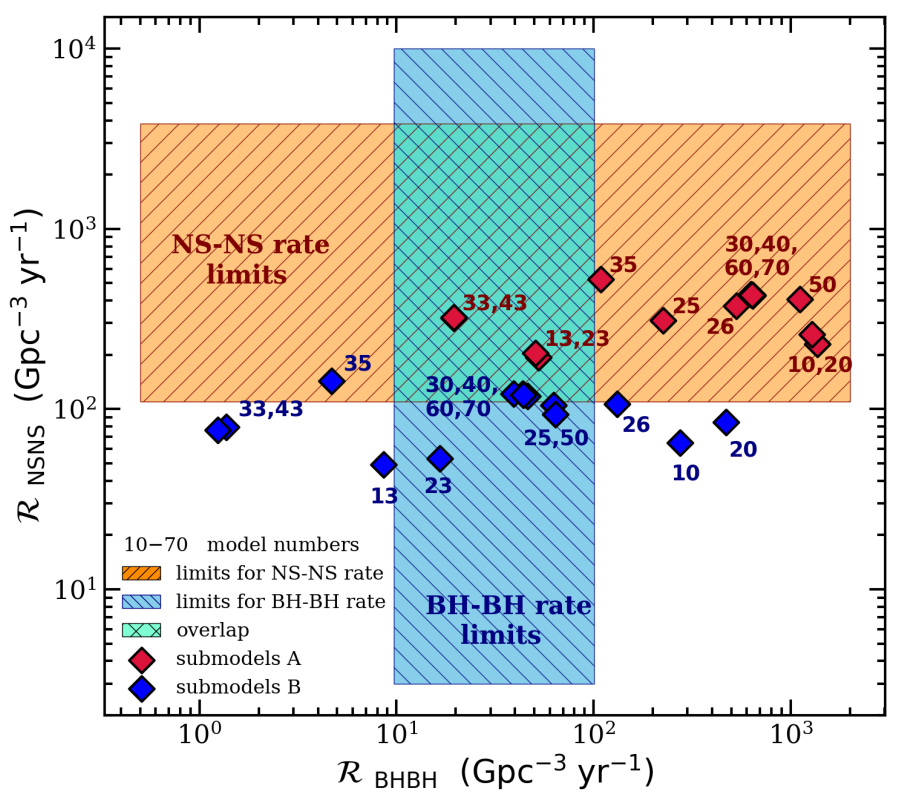

Fig. 24. Comparison of the local merger rate densities of $\mathrm{BH}-\mathrm{BH}$ and NS-NS mergers from all our models (see Table 3 ) with the current limits inferred from the O1/O2 LIGO/Virgo observational runs: 9.7-101 $\mathrm{Gpc}^{-3} \mathrm{yr}^{-1}$ for the BH-BH mergers and $110-3840 \mathrm{Gpc}^{-3} \mathrm{yr}^{-1}$ for the NS-NS events (Abbott et al. 2019b). Models that are consistent with the observational limits: M13.A, M23.A, M33.A, M43.A, M30.B, M40.B, M60.B, M70.B. We note that while some of the models are consistent with the observational constraints and others are not, it is not straightforward to draw conclusions about the physical ingredients of the models at this stage due to degeneracies in the impact of various assumptions on the theoretical merger rates (see Sect. 4.3.1).

them with $\mathrm{BH}-\mathrm{BH}$ merger rates obtained from dynamical evolution in globular clusters. In summary, the typical globular cluster rates $\left(5 \mathrm{Gpc}^{-3} \mathrm{yr}^{-1}\right)$ can explain about $10 \%$ of the LIGO/Virgo $\mathrm{BH}-\mathrm{BH}$ mergers (the empirical rate peaks at $\sim 50 \mathrm{Gpc}^{-3} \mathrm{yr}^{-1}$ ) while the remaining $90 \%$ of the observed rate can be easily explained by isolated binary BH-BH formation (see Table 3).

\subsubsection{Isolated binary evolution}

In this paper, we present a range of models and their rate densities. The rate density in each case is the result of several assumptions: starting from the cosmic star formation, metallicity evolution and continuing through the initial binary parameters and the binary evolution model up to the implied delay time (between the birth of a binary and the final merger of two compact objects) distribution. For each model, we calculated the local merger rate density $(z=0)$ as well as the predicted LIGO/Virgo detection rate in $\mathrm{O} 3$; see Table 3 . The local rate densities of BH-BH and NS-NS mergers that we obtained are directly comparable with the rate limits inferred by LIGO/Virgo at the conclusion of the $\mathrm{O} 1 / \mathrm{O} 2$ observing runs; see Fig. 24. Additionally, based on the recent LIGO/Virgo candidate (S190814bv) for a very likely ( $\sim 99 \%$ in the reported mass-based source classification) BH-NS merger from August 14 (LVC 2019a,b), we estimate the BH-NS merger rate density of $\mathcal{R}=1.6-60 \mathrm{Gpc}^{-3} \mathrm{yr}^{-1}$ (see Appendix A.9 for more), which can also be confronted with our models.

In addition to the overall rate of events, the relative rates of the three generic categories of events (BH-BH, BH-NS, NSNS) provide particularly valuable constraints. With this in mind, 


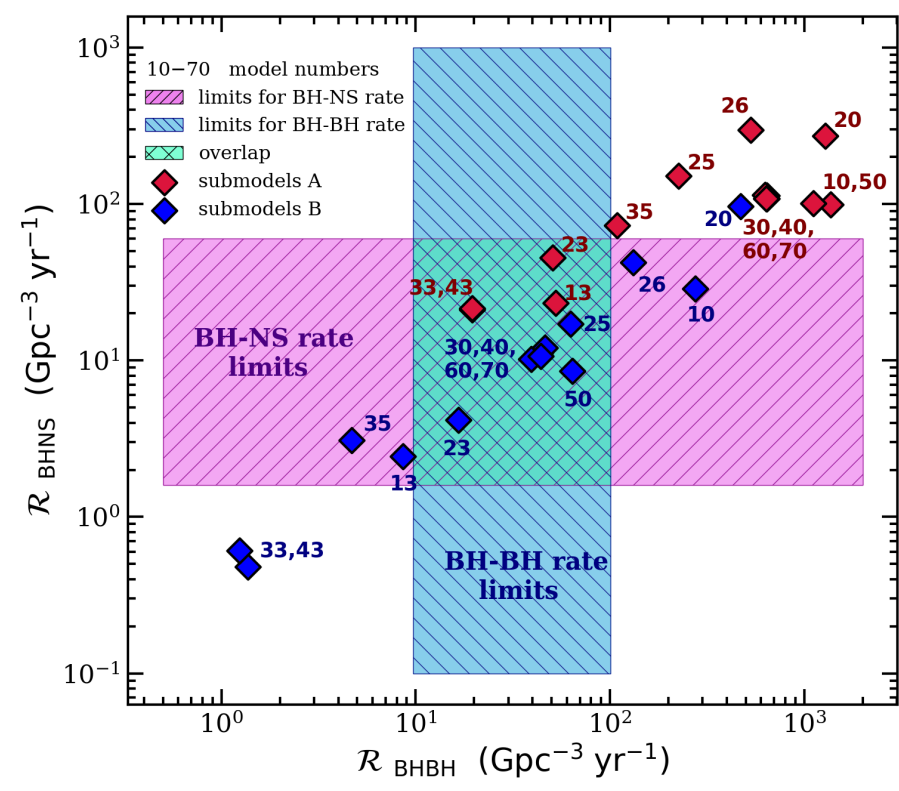

Fig. 25. Comparison of the local merger rate densities of $\mathrm{BH}-\mathrm{BH}$ and BH-NS mergers from all our models (see Table 3) with the current limits: $9.7-101 \mathrm{Gpc}^{-3} \mathrm{yr}^{-1}$ for the BH-BH mergers (LIGO/Virgo O1/O2) and 1.6-60 $\mathrm{Gpc}^{-3} \mathrm{yr}^{-1}$ for the BH-NS events (based on the LIGO/Virgo O3 candidate of the first BH-NS system, S190814bv, LVC 2019a,b; see Appendix A.9 for details). Models that are consistent with the observational limits: M13.A, M23.A, M33.A, M43.A, M23.B, M25.B M30.B, M40.B, M50.B, M60.B, M70.B. We also note that the BHNS merger rates from our models span the range between 0.48 and $297 \mathrm{Gpc}^{-3} \mathrm{yr}^{-1}$, which is consistent with the upper limit determined by their non-detection in O1/O2 LIGO/Virgo runs $\left(<610 \mathrm{Gpc}^{-3} \mathrm{yr}^{-1}\right.$, Abbott et al. 2019b).

in Figs. 24 and 25, we show the rates of all three source categories. The horizontal and vertical bands represent the allowed rates consistent with current LIGO/Virgo data. Superimposed on the LIGO/Virgo bands are the rate predictions from a range of our models, with the red diamonds from submodel A (the optimistic approach to common envelope, in which CE events with Hertzsprung gap donors are allowed to survive), and the blue diamonds from submodel B (the pessimistic approach to common envelope, in which CE events with Hertzsprung gap donors are not allowed to survive). We note that in both figures, there are many models that fall in the central sweet spot of the figure.

Looking at Fig. 24, we see that the existing $\mathrm{O} 1 / \mathrm{O} 2$ run bounds on the NS-NS and BH-BH merger rate densities already allow excluding some of the models. In the standard model group with the 2016 assumptions the only model consistent with the data is M13.A, which requires high $\mathrm{BH} / \mathrm{NS}$ natal kicks. If we allow some modified physics, we can also add model M23.A. From the group of models with updated physics of 2019, we can select two: M30.B ad M33.A, that are consistent with observations. Allowing for models with some modifications we can also include the models M40.B and M43.A, as well as M60.B and M70.B. Thus, we can see that the 2016 standard model is consistent with the merger rate density $\mathrm{O} 1 / \mathrm{O} 2$ runs data provided that the natal kicks are large. This may be in contradiction to some observations of $\mathrm{BH}$ in binaries that indicate small $\mathrm{BH}$ natal kicks, however, such a conclusion requires more detailed studies. The updated model of 2019 is consistent with the data. It also allows for certain modifications such as probing the origin of $\mathrm{BH}$ spins or the inclusion of various models of PPSNs.
The inclusion of the $\mathrm{BH}-\mathrm{NS}$ merger rate estimate (based on the $\mathrm{O} 1 / \mathrm{O} 2$ runs and ongoing $\mathrm{O} 3 \mathrm{run}^{6}$ ) (see Appendix A.9) in Fig. 25 offers additional insights. Of particular interest are models which fall in the central overlap region of both Figs. 24 and 25. For example, M13.A, M23.A, M33.A, M43.A, M30.B, M40.B, M60.B, and M70.B fall within the observed range for all three rates. A number of trends are evident from looking at both figures together. For example, we note that the B class of submodels, without the inclusion of HG donors in the CE evolution, brings many of the models into the center of the $\mathrm{BH}$ $\mathrm{BH} / \mathrm{BH}-\mathrm{NS}$ plot. However, this same change brings some of the models into tension or only into marginal agreement with the data on the BH-BH/NS-NS plot. In other words, submodel A is almost entirely excluded in Fig. 25, while submodel B is almost entirely excluded in Fig. 24. This might be a preliminary indication that the NS-NS systems do allow for CE with HG donors, while BH-NS systems do not. However, there are other factors that are relevant for the relative merger rates of different source types. For example, improving the metallicity evolution (which shifts the net star formation across the cosmic history towards higher metallicities) appears to shift down the BH-BH merger rates while leaving the NS-NS merger rates relatively unchanged (see also Chruslinska et al. 2019).

In all cases that we consider, the merger rate density increases with increasing redshift, peaks at about $z \approx 2$ and then decreases. A rough approximation of the scaling of the merger rate density with redshift in the range of $z \lesssim 2$ can be approximated as $\propto(1+z)^{1.5}$; however, the exponent is not wellconstrained. It is quite interesting to note that the assumed shape of the star formation rate history within the allowed bounds does not strongly influence the shape of the dependence of the merger rate density on redshift within $z \lesssim 2$; see Fig. 10 .

We expect that most of the remaining ambiguities should be resolved with the release of analysis of the $\mathrm{O} 3$ data. Assuming that these observations will yield about a hundred detections, we expect the bounds on the rates to narrow by a factor of three. This should allow us to strongly constrain the models presented in this paper based on the event rates alone. It should be stressed, however, that even then it will not be straightforward to constrain the underlying physics. Variations in different ingredients of a model can have a degenerate effect on the resulting merger rates. For instance, the inclusion of $\mathrm{HG}$ donors in the CE evolution (going from submodels B to A) leads to an increase in the merger rates, but so does the lowering of $\mathrm{BH} / \mathrm{NS}$ natal kicks (see Sect. 3.2 and Fig. 11 for details). The CE treatment and the natal kicks are only two of the many ingredients of our models, each of those ingredients being to some extent uncertain. This introduces a degeneracy that cannot easily be resolved at the moment. In the future, we can hope that the higher number of detected mergers allow us to combine all the inferred observables (e.g., rates, masses, spins, redshifts, host galaxies) in order to break the degeneracies and put better constraints on the model's input physics.

That said, submodels A: M13.A, M33.A, M43.A appear to be consistent with all rate estimates (although on the high end of the BH-NS limits). This shows the effect of the high natal kicks that reduce high $\mathrm{BH}-\mathrm{BH}$ merger rates typically found in the submodels A. At the same time, these high natal kicks do no affect the NS-NS merger rates significantly, as in our standard approach (fallback decreased kicks) NS natal kicks are already

6 Under assumption that the reported gravitational-wave signal from $\mathrm{S} 190814 \mathrm{bv}$ is in fact $\mathrm{BH}-\mathrm{NS}$ merger and not $\mathrm{BH}-\mathrm{BH}$ merger, both options being allowed. 
high. Submodels B: M30.B, M40.B, M60.B and M70.B, that appear consistent with all rate estimates, indicate that high average cosmic metallicity in unison with exclusion of CE events with HG donors may reduce high $\mathrm{BH}-\mathrm{BH}$ merger rates in comparison with older models and with submodels A. We note that these models include our current standard approach to input physics, with the two efficient angular momentum transport mechanisms (MESA and Fuller models) and the three prescriptions for PPSN (weak, moderate, and strong) mass loss. We note that some models are very close to being consistent with all rate constraints. Model M50.B is one such example. In this model, we reduce stellar winds to $30 \%$ of their standard values. Typically, BHs form with higher mass in this model. Additionally, with such low wind mass-loss rates for massive stars it is possible to form $\sim 60 M_{\odot}$ BHs in a solar metallicity environment $(Z=0.02)$ while still managing to avoid PPSN mass loss (see Belczynski et al. 2020 for discussion). At such mass-loss rates single stars (or stars in wide non-interacting binaries) may form $\sim 40 M_{\odot}$ He cores with $\sim 20-30 M_{\odot}$ H-rich envelopes leading to massive $\mathrm{BH}$ formation even at high metallicity. Such massive $\mathrm{BHs}$ would be found either as single BHs through microlensing surveys (e.g., Wyrzykowski \& Mandel 2020) or in wide binaries through radial velocity surveys (motion of the companion star). We note that such massive BHs would not necessarily add to either LIGO/Virgo or X-ray binary populations that form (at least in classical isolated binary evolution) from interacting binaries in which stars lose their H-rich envelopes (e.g., this study, Wiktorowicz et al. 2014). There is, of course, a complex interdependence between the various aspects of these models and this subset of models does not fully cover the parameter space. Nonetheless, these general trends may provide important clues about the underlying stellar and binary physics.

\subsubsection{Dynamical evolution in globular clusters}

In dense stellar environments, even binaries that are initially too wide to inspiral via gravitational radiation in a Hubble time can be hardened and induced to merge by binary-single and binarybinary interactions. Moreover, single BHs can segregate to the cluster center due to dynamical friction and form close binary systems that can merge due to gravitational radiation. Thus dense stellar systems such as globular clusters can be highly efficient per stellar mass at producing $\mathrm{BH}-\mathrm{BH}$ mergers. However, only a small fraction of stars are in globular clusters or similarly dense systems $\left(\sim 10^{-4}-10^{-3}\right.$, depending on the type of galaxy). This turns out to mean that the plausible rate density of mergers in dense stellar systems is low. A strong upper bound can be obtained (see Mandel \& Farmer 2018) by noting that there is roughly one globular cluster per $\mathrm{Mpc}^{3}$ in the local Universe. If each globular has $\sim 10^{6}$ stars (the actual average is a few times lower than this), $\sim 10^{-3}$ of stars become black holes, and all black holes pair up and merge in a Hubble time of $\sim 10^{10}$ years, then at most the rate will be $\sim 10^{6} \times 10^{-3} /\left(10^{10} \mathrm{yr} \times 1 \mathrm{Mpc}^{3}\right)$, or $\sim 100 \mathrm{Gpc}^{-3} \mathrm{yr}^{-1}$. In reality, the expected rate from clusters is ten to 100 times lower, for a discussion see Mandel \& Farmer (2018).

It does seem likely that globular cluster masses at formation were a few times larger than they are today. For example, it has been estimated that $\sim 60 \%-70 \%$ of cluster mass is lost in a Hubble time due to dynamical evolution and interactions with the host galaxy (Chatterjee et al. 2010; Giersz et al. 2013; Webb \& Leigh 2015). However, recent realistic estimates of $\mathrm{BH}-\mathrm{BH}$ merger rate densities from globular clusters are typically few $\mathrm{Gpc}^{-3} \mathrm{yr}^{-1}$ (Rodriguez et al. 2016c; Askar et al. 2017;
Park et al. 2017; Hong et al. 2018; Choksi et al. 2019). Recoil during binary-single and binary-binary interactions can be sufficient to eject a binary from its host globular, with the result that the majority of such mergers are expected to occur outside the globular (Portegies Zwart \& McMillan 2000 and many subsequent papers).

\subsection{BH masses}

An important test for population synthesis models is the direct comparison of their predictions of the mass distribution of binary black holes with the observed distribution from gravitational wave detections. The masses of black holes are among the best measured quantities of the LIGO/Virgo sources. In particular, the chirp mass is often measured to high accuracy $(\mathrm{O}(5 \%)$ or better) for lower mass mergers with a significant number of inspiral cycles detected in the LIGO/Virgo sensitivity band. For the higher mass systems, the total mass can be measured with reasonable accuracy. The individual component masses are often more poorly measured, and equivalently, the mass ratio is often relatively poorly constrained. For this discussion, we focus on the shape of the mass distribution.

As LIGO/Virgo continues to add to the sample of binary black holes, the inferred underlying mass distributions become increasingly well-constrained. Some preliminary limits have already been presented in Abbott et al. (2019a). In particular, that paper employs a number of different fits to the mass distribution of the more massive components of the detected binaries. For the purposes of comparison, we use the Model B from this paper, which consists of a power-law in $M_{\mathrm{BH} 1}$ with upper and lower mass cutoffs and a power-law in the binary mass ratio. The mass cutoff accounts for the dearth of high-mass black holes (Fishbach \& Holz 2017), as would be expected from PSN and PPSN (Belczynski et al. 2016c). LIGO/Virgo finds a power law index in the range of $\alpha=-3.1$ to $\alpha=0.1$ with peak probability at $\alpha=-1.6^{7}$, and with an upper mass cutoff between $36.3 M_{\odot}$ and $57 M_{\odot}$.

In Fig. 26, we show the intrinsic rate density of mergers averaged out to $z=0.5$ versus the mass of the more massive $\mathrm{BHs}$ in $\mathrm{BH}-\mathrm{BH}$ mergers $\left(M_{\mathrm{BH} 1}\right.$; upper figure) and the mass ratio $q=M_{\mathrm{BH} 2} / M_{\mathrm{BH} 1}$ (lower figure) for several of our models and compare them with the fits to Model B from Abbott et al. (2019a). Although Model B does not have the freedom to capture some of the features of the population synthesis distributions, the overall rate densities of the mass distribution found in the LIGO/Virgo collaboration analysis (shown in gray) are quite similar to those obtained, for example, in models M30.B, M40.B, and M50.B. We emphasize that the shapes of the primary mass distributions we obtain in these StarTrack models are better fit to exponentials than to power-laws and that there are some interesting features in the mass distributions at low BH mass. Nevertheless, compared to our theoretical models, the LIGO/Virgo Model B fits to the $10 \mathrm{O} 1 / \mathrm{O} 2 \mathrm{BH}-\mathrm{BHs}$ exhibit a similar falloff of the primary mass distribution towards higher masses, lower and upper mass cutoffs, and a falloff of the mass ratio distribution at more unequal masses.

At this point, we do not attempt any more elaborate fits to the $\mathrm{O} 1 / \mathrm{O} 2$ data than the comparison to the LIGO/Virgo phenomenological models, as these can wait till more LIGO/Virgo data points become available. For anyone interested in such fits, all our data is available online. With $\mathrm{O}(100) \mathrm{BH}-\mathrm{BH}$ mergers

\footnotetext{
We note that the LIGO/Virgo power law index is defined with a minus sign in front (see Eq. (2) of Abbott et al. 2019a).
} 

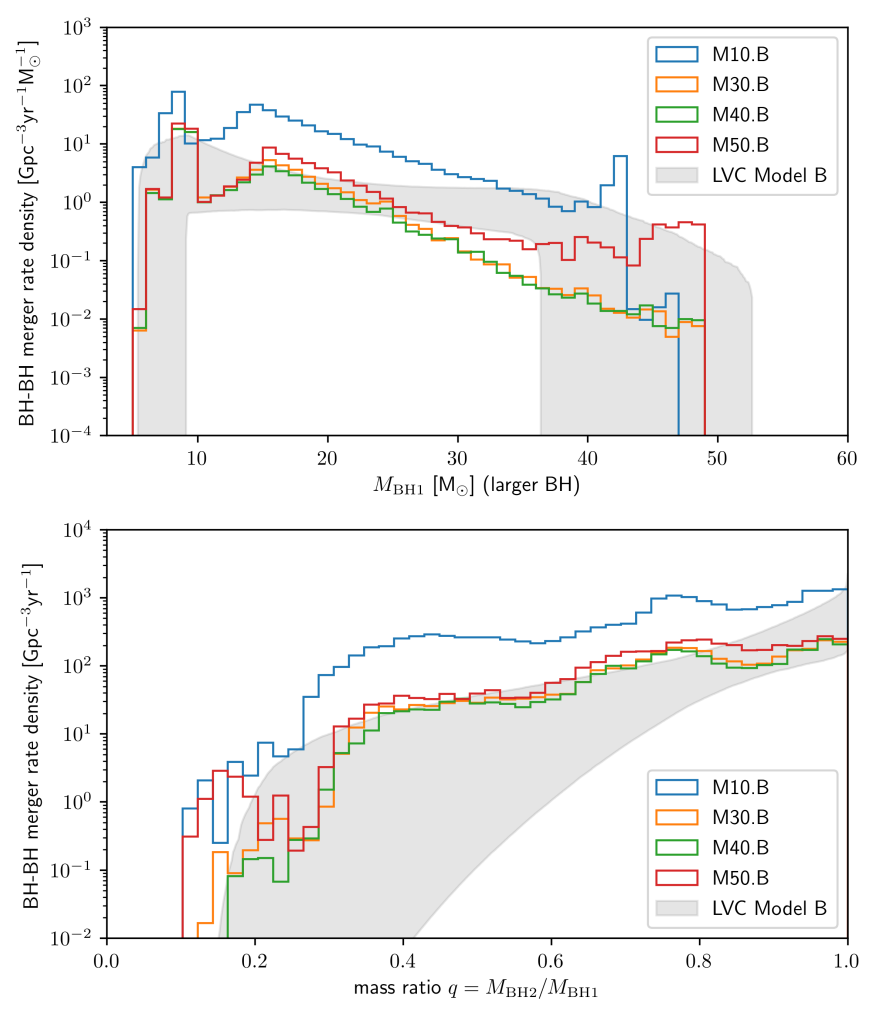

Fig. 26. Intrinsic merger rate distribution as a function of primary black hole mass $M_{\mathrm{BH} 1}$ (top) and binary mass ratio $M_{\mathrm{BH} 2} / M_{\mathrm{BH} 1}$ of $\mathrm{BH}-\mathrm{BH}$ mergers (bottom) for different StarTrack model choices. The gray bands show the $90 \%$ confidence limits on the populations inferred in Abbott et al. (2019a) using the phenomenological Model B.

expected for the entirety of $\mathrm{O} 3$, we will be able to test the existence of some of the finer features seen in our mass distributions as well as the goodness-of-fit of exponentials versus power-laws to the primary masses.

\subsection{BH-BH effective spins}

The rate of $\mathrm{BH}-\mathrm{BH}$ mergers with different effective spins provides several reliable features that we can use to corroborate and constrain our models with present and future GW observations.

First and foremost, as has been discussed in our and other previous work, our binary evolution models only rarely, if ever, produce binaries with $\chi_{\text {eff }}$ significantly below zero (see Figs. 18-21). This void provides an opportunity to identify the unique contribution from alternative formation channels as our models cannot produce a preponderance of events with negative effective spins.

Second, our predicted effective spin distributions have sharp cutoff-like features in each of the one (or sometimes two) subpopulations that dominate the detection rate versus effective spin. For example, the black hole spin distribution drops sharply for $\chi_{\text {eff }} \gtrsim 0.9$ in the inefficient Geneva angular momentum transport mechanism (Fig. 18), above 0.2 for the binary black holes not spun up by tides in the MESA models; above 0.6 for the subpopulation of BH-BH with a tidally-spun-up WR progenitor in the MESA models (Fig. 19); and near 0.1 for the Fuller models (Fig. 20). Sharp cutoffs like these are rapidly identified empirically, allowing future gravitational-wave observations to constrain the BH natal spin distribution, and the physics of tidal spinup.
Third, for models with WR tides (e.g., M30.B and M40.B shown in Figs. 19 and 20), our effective spin distribution has two well-separated subpopulations (a peak and a tail), associated with the principal channel and the WR-spinup channel. In other words, the gravitational-wave population may allow us to reliably associate specific binary physics to specific binary black hole mergers, and thus to measure the relative proportions (and properties) of BH-BH systems forming through each channel.

While our binary evolution models have many parameters, because of the limited role of accretion on the BH spin, very few parameters have more impact on the $\chi_{\text {eff }}$ distribution than the physics we have described above. For example, we show with Fig. 22 that BH kicks have a relatively modest effect on the shape of the $\chi_{\text {eff }}$ distribution. Strong BH kicks can for example make the $\chi$ eff distribution more symmetric and isotropic, effectively by disrupting binaries which would otherwise dominate the sharp cutoffs at the largest values of $\chi_{\text {eff }}$. In other words, these kicks make the $\chi_{\text {eff }}$ merger rate smaller and with a less prominent (and therefore more difficult to identify) cutoff. Although this poorly constrained physics of natal kicks can reduce the rate at which $\chi_{\text {eff }}$ measurements can inform about the binary evolution and diminish the contrast of the features we have described above, they cannot erase them, particularly with regard to features such as the second population of WR tidally spun-up stars or the low probability of mergers with large negative $\chi_{\text {eff }}$.

In Fig. 27, we show the cumulative density functions (CDFs) of the effective $\operatorname{spin} \chi_{\text {eff }}$ for a select set of our models and compare them to the $\chi$ eff measured for the $10 \mathrm{O} 1 / \mathrm{O} 2 \mathrm{BH}-\mathrm{BH}$ mergers. For each model we show the $\chi$ eff $\mathrm{CDF}$ of detectable events as a color solid line. In the corresponding colored shaded regions, we show the $90 \%$ range of 5000 mock observed CDFs under each model. To generate a mock observed CDF, we draw $10 \chi_{\text {eff }}$ values from the detected population under a given model and add random Gaussian noise with $\sigma=0.05$, which is approximately the uncertainty in $\chi_{\text {eff }}$ likelihoods from the events of GWTC-1 (we resample any samples with $\mid \chi$ eff $\mid>1$ after adding noise). The black line shows the $\chi_{\text {eff }}$ CDF of median likelihoods of the $\mathrm{O} 1 / \mathrm{O} 2 \mathrm{LVC}$ events, and the gray region bounds CDFs of the 5 th and 95th percentiles of the likelihoods from LVC parameter estimation. Notably, these effective spins are clustered around zero effective spin, indicating that model M10.B's predicted spins are disfavored by the data. Models M30.B (no WR tides), M30.B (WR tides), and M40.B are much more consistent with these observations, and with the LIGO/Virgo collaboration analysis in Abbott et al. (2019a), which finds a preference for most effective spins to be very close to zero (see Fig. 12 in Abbott et al. 2019a). That analysis finds that only a few tens of percent of the observed BH-BH mergers have $\left|\chi_{\text {eff }}\right|>0.05$, and of those with $\left|\chi_{\text {eff }}\right|>0.05$, almost all are likely to have positive $\chi_{\text {eff }}$. For the moment the observed $\chi_{\text {eff }}$ distribution does not allow to test the importance of WR star tides during the evolution towards a $\mathrm{BH}$ even if it seems to slightly favor the no-tide path. Overall, the limited $\mathrm{BH}-\mathrm{BH}$ sample from $\mathrm{O} 1 / \mathrm{O} 2$ does not strongly constrain the $\mathrm{BH}$ spins other than to the conclusion that the effective spins tend to be near zero. However, it suggests that O3 should provide data that will allow selecting evolutionary path that lead to BH-BH mergers.

\subsection{Dichotomy of LIGO/Virgo BHs and HMXB BHs}

LIGO/Virgo BHs are mostly massive (see Table 1) and it seems that the spins of these BHs are small. Unfortunately, the spin magnitudes of massive BHs with $M_{\mathrm{BH}}>15 M_{\odot}$ are not constrained by other observational means. For example, the 

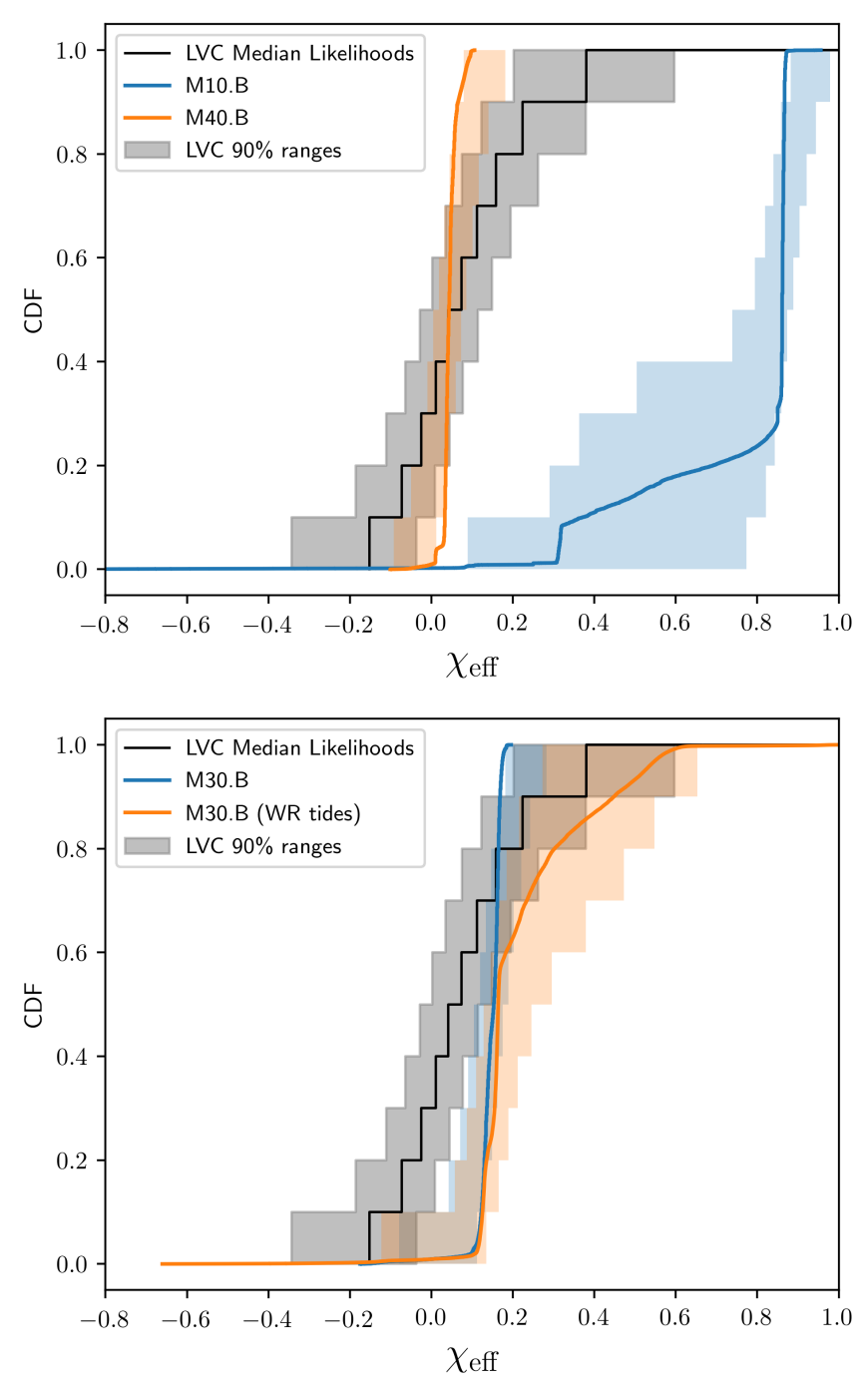

Fig. 27. Cumulative density functions (CDFs) of four StarTrack models versus effective spins $\chi_{\text {eff }}$. Solid color lines correspond to $\chi_{\text {eff }}$ CDF of detectable events for each model. The black line shows the $\chi_{\text {eff }}$ $\mathrm{CDF}$ of median likelihoods of the $\mathrm{O} 1 / \mathrm{O} 2 \mathrm{LVC}$ events, and the gray region bounds CDFs of the 5th and 95th percentiles of the likelihoods from LVC parameter estimation. In the corresponding colored shaded regions, we show the $90 \%$ range of 5000 mock observed CDFs under each model. To generate a mock observed CDF, we draw $10 \chi_{\text {eff }}$ values from the detected population under a given model and add random Gaussian noise with $\sigma=0.05$, which is approximately the uncertainty in $\chi_{\text {eff }}$ likelihoods from the events of GWTC-1 (we resample any samples with $\left|\chi_{\text {eff }}\right|>1$ after adding noise).

three most massive BHs in wind-fed high mass X-ray binaries (HMXB) for which we have spin estimates are at roughly $1 / 3$ or $1 / 2$ the mass of the larger-mass LIGO/Virgo BHs. The estimated masses and spins of these BHs can be found online $\mathrm{at}^{8}$ : $\mathrm{LMC} \mathrm{X}-1, M_{\mathrm{BH}}=10.9 \pm 1.6 M_{\odot}\left(a_{\text {spin }}=0.92\right) ; \mathrm{Cyg} \mathrm{X}-1, M_{\mathrm{BH}}=$ $14.8 \pm 0.1 M_{\odot}\left(a_{\text {spin }}>0.983\right) ; \mathrm{M} 33 \mathrm{X}-7, M_{\mathrm{BH}}=15.7 \pm 1.5 M_{\odot}$ $\left(a_{\text {spin }}=0.84\right)$. We note that BH spins can be measured by two methods: disk reflection and disk continuum. For LMC-1 and Cyg X-1 BH spins from both methods are consistent, while for M33 X-7 BH spin was estimated only through disk continuum (Miller \& Miller 2015). There is an apparent tension between the spin estimates of LIGO/Virgo BHs and BHs in these HMXBs. However, this tension can be possibly avoided if HMXBs and

\footnotetext{
8 https://universeathome.pl/universe/blackholes.php
}

LIGO/Virgo systems form through different evolutionary scenarios.

First, none of these three HMXBs is expected to produce a $\mathrm{BH}-\mathrm{BH}$ merger. Future evolution of these systems was studied and it was shown that none of these systems will form a $\mathrm{BH}$ BH merger. They will either end up as single objects (in which $\mathrm{BH}$ merges with its massive companion star), or at best they may form BH-NS systems (Belczynski et al. 2012b). We note that this conclusion was reached in the framework of the classical binary evolution that does not take into account homogeneous evolution of rapidly rotating stars that allows for the formation of $\mathrm{BH}-\mathrm{BH}$ mergers in alternative ways (Marchant et al. 2016; Mandel \& de Mink 2016; de Mink \& Mandel 2016).

Second, it was argued that HMXBs and LIGO/Virgo systems may form through different evolutionary scenarios. As explained in the past, the classical isolated binary evolution channel forms $\mathrm{BH}-\mathrm{BH}$ mergers from (initially) very wide binaries $\left(a \gtrsim 1000 R_{\odot}\right.$; see Figs. 8 or 9 or de Mink \& Belczynski 2015; Belczynski et al. 2016a). Therefore, if the tidal spin-up operates, it only acts at the very end of the evolution during the BH-WR stage (potentially) spinning up some fraction of the second-born BHs in BH-BH mergers (see Sects. 2.5 and 3.4). This makes the majority of $\mathrm{BHs}$ in $\mathrm{BH}-\mathrm{BH}$ mergers to have low spins if efficient angular momentum transport is adopted for massive stars (as in our MESA or Fuller models). On the other hand, HMXBs were proposed to form from initially very close binaries $\left(a \sim 100 R_{\odot}\right.$, e.g., Valsecchi et al. 2010; Qin et al. 2019). In such scenario a system with a massive main sequence primary is tidally locked so, through its evolution, its spin is kept high at the expense of the orbital angular momentum. At such small orbital separations the binary undergoes case A mass transfer or homogeneous evolution that keeps reducing H-rich envelope of the donor primary and keeps it from expanding. After main sequence evolution, the primary is a compact WR star that quickly collapses to a $\mathrm{BH}$. The $\mathrm{BH}$ is found in the near proximity of its companion star (if natal kick is small), naturally producing a (wind-fed) HMXB. Possibly, tidal interactions can keep the primary spin high until the end of its nuclear evolution which would lead to the formation of a rapidly spinning BH. However, the detailed evolutionary calculations have shown that if efficient (Tayler-Spruit dynamo) angular momentum transport is assumed for the primary star, then properties of the three HMXBs considered here cannot be reproduced (Qin et al. 2019). Therefore, the tension still exists, albeit it seems like HMXBs and LIGO/Virgo $\mathrm{BH}-\mathrm{BH}$ mergers originate from different initial populations of binaries (close versus wide) but specific details of evolution leading to the formation of HMXBs need to be worked out.

We note that formation scenarios that require rapidly spinning stars (whether they lead to formation of $\mathrm{BH}-\mathrm{BH}$ mergers or BH HMXBs) are not available in our study. Rapid rotation induces efficient mixing and reduces radial expansion of stars in homogeneous or semi-homogeneous evolution. Our calculations can only be applied to slow- or moderately-rotating stars in classical binary evolution. Both binary channels (homogeneous and classical) do not exclude one another, but rather they both operate in Universe. Finally, we should stress that estimates of $\mathrm{BH}$ spins through properties of their accretion disks are not direct spin measurements but they are model-dependent, so there is still a possibility that the tension is only apparent.

\subsection{Origin of $\mathrm{LIGO}$ /Virgo $\mathrm{BHs}$}

At the moment (only O1/O2: data available), it seems that the classical isolated binary evolution formation channel is capable 
of explaining all the basic properties of the LIGO/Virgo BHBH mergers. This does not mean than other channels do not contribute to LIGO/Virgo detections. For example, if we compare the average rate estimates of the isolated binary channels (see Table 3) and the dynamical formation channels in globular clusters (see Sect. 4.3.2), it appears that 1 in $10 \mathrm{BH}-\mathrm{BH}$ mergers might come from a globular cluster. There may be yet another dynamical contribution from young open clusters to BHBH formations (Ziosi et al. 2014; Di Carlo et al. 2019). Even the homogeneous isolated binary channel is not excluded by the observed LIGO/Virgo low BH-BH effective spins, as apparently BHs with a broad range of spins $\left(a_{\text {spin }} \sim 0.2-1\right)$ can be produced also in this scenario (Ph. Podsiadlowski, priv. comm.). In the published models with rather sparse metallicity sampling and conservative evolutionary assumptions (e.g., no pair-instability pulsation supernova mass $\left.\operatorname{loss}{ }^{9}\right)$, BH spins are found in somewhat narrower range: $a_{\text {spin }} \sim 0.4-1$ for $M_{\mathrm{BH}} \lesssim 60 M_{\odot}$ (e.g., see Fig. 9 of Marchant et al. 2016).

If a $\mathrm{BH}-\mathrm{BH}$ merger is discovered with either of the binary components in the mass range $70 / 80 \lesssim M_{\mathrm{BH}} \lesssim 135 M_{\odot}$ with a high spin of $a_{\text {spin }} \approx 0.7$, this would strongly suggest a dynamical formation scenario. The lower-mass limit corresponds to the pair-instability pulsation supernova effects on the presupernova star and on the remnant BH mass (Woosley 2017; Limongi \& Chieffi 2018; Belczynski et al. 2020), while the upper mass limit corresponds to the end of the pair-instability supernova process, that is believed to disrupt the entire star without BH formation (Fryer et al. 2001; Heger \& Woosley 2002). The lack of $\mathrm{BHs}$ in this mass range is referred to as the "second mass gap" (Belczynski et al. 2014; Spera et al. 2015; Marchant et al. 2016; Fishbach \& Holz 2017). It seems unlikely that isolated binaries can fill this gap, but repeated BH-BH mergers in dense environments could produce such heavy BHs, and it is expected that they would have moderately high spins $a_{\text {spin }} \approx 0.7$ (Gerosa \& Berti 2017; Fishbach et al. 2017). However, it cannot be excluded that a $\mathrm{BH}$ with high mass and low spin is formed by a merger of two BHs (Belczynski \& Banerjee 2020). Alternatively, the detection of a low-spin $\mathrm{BH}$ with mass within the second mass gap may point to either (i) inconsistencies in pair-instability supernova theory or (ii) a primordial BH origin (Green 2017). There is also an issue of the second mass gap width. The lower bound may be as high as $70-80 M_{\odot}$ depending on details of input physics in stellar models (Limongi \& Chieffi 2018; Belczynski et al. 2020). The upper bound may change if fusion reaction rates of heavy elements that are involved in pair-instability supernovae change. We note that the reaction rates are uncertain (Fields et al. 2018). Possibly, the second mass gap is narrower than currently believed.

In the classical isolated binary evolution channel, we show that if LIGO/Virgo observations of BH-BH mergers remain consistent with small effective spins $\chi_{\text {eff }} \simeq 0$, this would indicate that low natal spins are common in BHs. In this case, our work shows that stellar models with mild rotational coupling between the stellar interior (core) and the outer zones (envelope) would be disfavored. Our main finding is that angular momentum transport in massive stars (so far unconstrained by the electromagnetic observations) is more efficient than predicted by the Geneva shellular model. This demonstrates how LIGO/Virgo observations can be used to make clear astrophysical inferences and guide stellar evolution astrophysics. Our conclusion is not subject to the main known population synthesis uncertainties, since

\footnotetext{
9 We note that pair-instability pulsation supernova mass loss, if taken into account, can reduce spin by about 30\% (Marchant et al. 2019).
}

we have explored a broad range of key parameters: $\mathrm{BH}$ natal kicks, initial star rotation, tides, spin-up by accretion onto BHs.

We note that adjusting the angular momentum transport to produce low spinning BHs in BH-BH mergers, to fit LIGO/Virgo observations leads to several astrophysical inferences and constraints on massive binary evolution. (i) RLOF between two massive stars (see second evolutionary stage in $\mathrm{BH}-\mathrm{BH}$ formation; Fig. 8 or 9) cannot effectively spin up the core of the accreting MS star (since this would produce a $\mathrm{BH}$ with large spin). This can be avoided if the RLOF is highly non-conservative and not much mass is accreted onto MS star (this is not excluded by any EM observations). We note that we have assumed that $50-80 \%$ of the mass is lost in RLOF, but in our models this fraction can be easily increased. Alternatively, if accretion is significant and if the entire star is spun-up; then we obtain an additional constraint on the angular momentum transport. It needs to be effective enough to remove most of the angular momentum from the highly spinning core to the envelope in about $1 \mathrm{Myr}$ (time between RLOF and CE which expels the envelope from the binary). (ii) Spin-up of the first-formed $\mathrm{BH}$ by accretion in the $\mathrm{CE}$ phase must be negligible as predicted by recent studies (MacLeod et al. 2017; Murguia-Berthier et al. 2017; Holgado et al. 2018, see the fifth evolutionary stage in BH-BH formation; Figs. 8 and 9). (iii) Tidal torques are not effective in BH-WR binaries that form $\mathrm{BH}-\mathrm{BH}$ mergers as the WR star spin-up would lead to the formation of a highly spinning $\mathrm{BH}$ (see the sixth evolutionary stage in BH-BH formation; Figs. 8 and 9). In our evolution, a small, but significant fraction ( 20-30\%; see Figs. 19 and 20) of the BH-WR/WR-BH/WR-WR binaries are found on orbits smaller than $\sim 10-20 R_{\odot}$ (orbital periods $P_{\text {orb }}<1.3 \mathrm{~d}$ ) for which the tides are expected to be effective for WR stars (Kushnir et al. 2016; Zaldarriaga et al. 2018; Qin et al. 2018). Therefore if there are no detections of highly spinning BHs in LIGO/Virgo observations it will imply that tides are not as effective as argued by recent work or that $\mathrm{BH}-\mathrm{BH}$ mergers are not produced by the isolated classical binary evolution (Hotokezaka $\&$ Piran 2017). We note, however, that already one event so far (GW170729) may have an effective spin as high as $\chi_{\text {eff }}=0.57$ ( $90 \%$ credible limits; see Table 1). On the other hand, among ten events, it can be expected that one outlier will appear within the $90 \%$ credible limits. Therefore, at the moment the comparison of models with observations remains inconclusive with respect to tides. Yet, the comparison of models predicting tidally spunup stars, producing highly spinning $\mathrm{BHs}$, with the number of LIGO/Virgo high effective spin $\mathrm{BH}-\mathrm{BH}$ mergers may help to constrain the strength of tides in near future.

Finally, we note that our main conclusion, which states that angular momentum transport needs to be more effective than predicted by shellular model for massive stars, lends support to a similar conclusion that was reached for low-mass stars based on Kepler asteroseismology data (Cantiello et al. 2014).

\section{Conclusions}

In this work, we updated our method of population synthesis calculations with revised and extended input physics that is important for the formation of double compact object mergers: BH-BH, BH-NS, and NS-NS. We introduced new models of pairinstability pulsation supernovae that are crucial in mass estimates of heavy black holes. We employed the recent estimates of starformation rate density and cosmic metallicity evolution in our calculations. These two factors play an important role in setting the double compact object merger rates (Belczynski et al. 2010b; Dominik et al. 2013; Chruslinska et al. 2019; Neijssel et al. 2019). 
We introduced new models for $\mathrm{BH}$ natal spin that allow for a direct comparison with $\mathrm{LIGO} /$ Virgo estimates of the effective spin parameter of $\mathrm{BH}-\mathrm{BH}$ mergers. These models connect gravitational-wave observations with detailed stellar evolution calculations of angular momentum transport in massive stars that is otherwise hidden from electromagnetic observations. We also allowed for significantly decreased stellar winds with respect to the standard wind prescriptions (Vink et al. 2001) used in modeling. Winds are an important factor that sets the shape of black hole mass distribution. Finally, updated prescriptions of accretion onto compact objects in stable Roche-lobe overflow, common envelope, and from stellar winds were used in our calculations.

We summarize our main findings in the following points.

1. Our study is the first to employ detailed single stellar evolutionary calculations in binary population synthesis to compare LIGO/Virgo BH-BH effective spins with those resulting from several angular momentum transport mechanisms. If LIGO/Virgo BH-BH mergers originate from the classical isolated binary evolution channel, then their low effective spins inform about the effective angular momentum transport in massive stars and most likely disfavor effective tidal interactions in close binaries with WR stars. According to our evolutionary framework, the Tayler-Spruit magnetic dynamo (as implemented in the MESA or in the Fuller model) reproduces the effective spin measurements very well, while meridional currents (as implemented in the Geneva model) are inconsistent with the current LIGO/Virgo data.

2. For some of our models, the predicted merger rate densities of BH-BH, BH-NS, and NS-NS systems are within the LIGO/Virgo empirical estimates. The rates strongly depend on the cosmic metallicity evolution, the choice of NS/BH natal kicks and the treatment of the common envelope phase. Due to the similar effects of these factors on the rates, it still is not possible to derive definitive conclusions about any of these pieces of input physics individually. However, some combinations of parameters may be already excluded. Interparameter degeneracies make up an important factor that cannot be overlooked in deriving astrophysical conclusions from gravitational wave observations.

3. The range of the observed $\mathrm{BH}$ masses appears to be in agreement with all three of our adopted PPSN models: from strong PPSN with maximum $\mathrm{BH}$ mass of $M_{\mathrm{BH}, \max } \sim 40 M_{\odot}$, to moderate PPSN with $M_{\mathrm{BH} \text {,max }} \sim 50 M_{\odot}$, and to weak PPSN with $M_{\mathrm{BH}, \max } \sim 55 M_{\odot}$. If heavier BHs are observed it will indicate that, either the pair-instability pulsation supernovae and the pair-instability supernovae do not work as predicted, or that BHs with very heavy mass originate from other formation channels. The values of BH spins may distinguish these two possibilities. If a massive $\mathrm{BH}\left(\sim 100 M_{\odot}\right)$ is observed with low spin it will indicate a classical isolated binary evolution formation channel, but such an observation will cast shadow on PPSN/PSN predictions. If, however, such a $\mathrm{BH}$ is found to have a large spin as expected from consecutive $\mathrm{BH}$ mergers, then its presence will point to formation in a dense stellar environment (e.g., globular cluster).

4. Based on our limited set of models and the initial ten $\mathrm{O} 1 / \mathrm{O} 2$ detections, if we were to make a set of statements on the physics of massive binaries, we could venture to say that with our revised cosmic metallicity evolution, it seems that to simultaneously reproduce the observed $\mathrm{BH}-\mathrm{BH}, \mathrm{BH}-\mathrm{NS}$, and NS-NS merger rates one should use submodels B (no $\mathrm{CE}$ allowed with $\mathrm{HG}$ donors) if $\mathrm{BH}$ kicks are low, but if these kicks are high, submodels A (CE allowed with HG donors) should be preferred. If individual $\mathrm{BH}$ masses are in fact as high as LIGO/Virgo reports for the most likely values from O1/O2 run (e.g., $50.6 M_{\odot}$ for GW170729) then we can already exclude strong mass ejection during the PPSN.

We note that similar conclusions have been reached by Bavera et al. (2020) and Neijssel et al. (2019), although these authors used somewhat different approaches to their detailed stellar evolution models and a different population synthesis code was used.

Acknowledgements. We have benefited from comments from Selma de Mink, Enrico Ramirez-Ruiz, Ilya Mandel, Thomas Janka, Serena Repetto, Simon Stevenson, Sambaran Banerjee, Tom Maccarone, Craig Heinke, Phil Charles, Doron Kushnir, Tsvi Piran, Miguel Holgado, Antonio Claret, Tassos Fragos, Philipp Podsiadlowski, and Matt Benacquista. We would like to thank thousands of Universe@home users that have provided their personal computers and phones for our simulations, and in particular to Krzysztof Piszczek (program IT managers). KB acknowledges support from the Polish National Science Center (NCN) grant Maestro (2018/30/A/ST9/00050) and KB, SM and JPL from the NCN grant OPUS (2015/19/B/ST9/01099). JK and MC acknowledge support from the Netherlands Organisation for Scientific Research (NWO). C.E.F. acknowledges support from a Pre-doctoral Fellowship administered by the National Academies of Sciences, Engineering, and Medicine on behalf of the Ford Foundation, an Edward J Petry Graduate Fellowship from Michigan State University, and the National Science Foundation Graduate Research Fellowship Program under grant number DGE1424871. MG ans AA were partially supported by the National Science Centre, Poland, through the grant UMO2016/23/B/ST9/02732. This work has been supported by the EU COST Action CA16117 (ChETEC). RH, KN, KB acknowledge support from the World Premier International Research Centre Initiative (WPI Initiative), MEXT, Japan. D.G. is supported by Leverhulme Trust Grant No. RPG-2019-350 and by NASA through Einstein Postdoctoral Fellowship Grant No. PF6-170152 awarded by the Chandra X-ray Center, which is operated by the Smithsonian Astrophysical Observatory for NASA under contract NAS8-03060. TB acknowledges support from the Polish National Science Center (NCN) Grant No. UMO2014/14/M/ST9/00707. EB is supported by NSF Grants No. PHY-1607130, AST-1716715, and by FCT contract IF/00797/2014/CP1214/CT0012 under the IF2014 Programme. C.G., G.M., and S.E. acknowledge support from the Swiss National Science Foundation (project number 200020-160119). ROS is supported by NSF AST-1412449, PHY-1505629, and PHY-1607520. DAB acknowledges support from National Science Foundation Grant No. PHY-1404395. G.W. is partly supported by the President's International Fellowship Initiative (PIFI) of the Chinese Academy of Sciences under grant no.2018PM0017 and by the Strategic Priority Research Program of the Chinese Academy of Science Multiwaveband Gravitational Wave Universe (Grant No. XDB23040000). AA is supported by the Carl Tryggers Foundation for Scientific Research through the grant CTS 17:113. MCM acknowledges support by NASA grant 80 NSSC18K0527. This research was supported in part by the National Science Foundation under Grant No. NSF PHY-1748958 to KITP UC Santa Barbara. Several authors (KB EB, CLF, DAB, DEH, RO, KN, MCM, GM, JPL) were supported by KITP UC Santa Barbara to work on this project. JPL was supported by a grant from the French Space Agency CNES. S.C.L acknowledges support by the funding HSTAR-15021.001-A. DEH was partially supported by NSF grant PHY-1708081, and also by the Kavli Institute for Cosmological Physics at the University of Chicago through an endowment from the Kavli Foundation. He also gratefully acknowledges support from a Marion and Stuart Rice Award. We thank the Niels Bohr Institute for its hospitality while part of this work was completed, and acknowledge the Kavli Foundation and the DNRF for supporting the 2017 Kavli Summer Program.

\section{References}

Abbott, B. P., et al. (LIGO Scientific and Virgo Collaboration) 2017, Phys. Rev. Lett., 118, 221101

Abbott, B. P., Abbott, R., Abbott, T. D., et al. 2019a, ApJ, 882, L24

Abbott, B. P., Abbott, R., Abbott, T. D., et al. 2019b, Phys. Rev. X, 9, 031040

Abolmasov, P., Karpov, S., \& Kotani, T. 2009, PASJ, 61, 213

Abt, H. A., Gomez, A. E., \& Levy, S. G. 1990, ApJS, 74, 551

Andrews, J. J., \& Zezas, A. 2019, MNRAS, 486, 3213

Antonini, F., Toonen, S., \& Hamers, A. S. 2017, ApJ, 841, 77

Antonini, F., Rodriguez, C. L., Petrovich, C., \& Fischer, C. L. 2018, MNRAS, 480, L58

Arca-Sedda, M., \& Capuzzo-Dolcetta, R. 2019, MNRAS, 483, 152

Askar, A., Szkudlarek, M., Gondek-Rosińska, D., Giersz, M., \& Bulik, T. 2017, MNRAS, 464, L36

Bae, Y.-B., Kim, C., \& Lee, H. M. 2014, MNRAS, 440, 2714 
Banerjee, S. 2018, MNRAS, 473, 909

Bastian, N., Covey, K. R., \& Meyer, M. R. 2010, ARA\&A, 48, 339

Bavera, S. S., Fragos, T., Qin, Y., et al. 2020, A\&A, 635, A97

Belczynski, K., \& Banerjee, S. 2020, ArXiv e-prints [arXiv:2002.08050]

Belczynski, K., \& Taam, R. E. 2004, ApJ, 603, 690

Belczynski, K., Kalogera, V., \& Bulik, T. 2002, ApJ, 572, 407

Belczynski, K., Taam, R. E., Kalogera, V., Rasio, F. A., \& Bulik, T. 2007, ApJ, 662,504

Belczynski, K., Taam, R. E., Rantsiou, E., \& van der Sluys, M. 2008a, ApJ, 682, 474

Belczynski, K., Kalogera, V., Rasio, F. A., et al. 2008b, ApJS, 174, 223

Belczynski, K., Bulik, T., Fryer, C. L., et al. 2010a, ApJ, 714, 1217

Belczynski, K., Dominik, M., Bulik, T., et al. 2010b, ApJ, 715, L138

Belczynski, K., Wiktorowicz, G., Fryer, C. L., Holz, D. E., \& Kalogera, V. 2012a, ApJ, 757, 91

Belczynski, K., Bulik, T., \& Fryer, C. L. 2012b, ArXiv e-prints [arXiv:1208.2422]

Belczynski, K., Buonanno, A., Cantiello, M., et al. 2014, ApJ, 789, 120

Belczynski, K., Holz, D. E., Bulik, T., \& O’Shaughnessy, R. 2016a, Nature, 534, 512

Belczynski, K., Repetto, S., Holz, D. E., et al. 2016b, ApJ, 819, 108

Belczynski, K., Heger, A., Gladysz, W., et al. 2016c, A\&A, 594, A97

Belczynski, K., Hirschi, R., Kaiser, E. A., et al. 2020, ApJ, 890, 113

Benacquista, M. J., \& Downing, J. M. B. 2013, Liv. Rev. Relativ., 16, 4

Berg, D. A., Skillman, E. D., Marble, A. R., et al. 2012, ApJ, 754, 98

Blondin, J. M., \& Mezzacappa, A. 2007, Nature, 445, 58

Bouret, J. C., Lanz, T., Hillier, D. J., et al. 2015, MNRAS, 449, 1545

Cantiello, M., Mankovich, C., Bildsten, L., Christensen-Dalsgaard, J., \& Paxton, B. 2014, ApJ, 788, 93

Chatterjee, S., Fregeau, J. M., Umbreit, S., \& Rasio, F. A. 2010, ApJ, 719, 915

Chatterjee, S., Rodriguez, C. L., Kalogera, V., \& Rasio, F. A. 2017, ApJ, 836 L26

Chini, R., Hoffmeister, V. H., Nasseri, A., Stahl, O., \& Zinnecker, H. 2012 MNRAS, 424, 1925

Choksi, N., Volonteri, M., Colpi, M., Gnedin, O. Y., \& Li, H. 2019, ApJ, 873, 100

Chrimes, A. A., Stanway, E. R., \& Eldridge, J. J. 2020, MNRAS, 491, 3479

Chruslinska, M., \& Nelemans, G. 2019, MNRAS, 488, 5300

Chruslinska, M., Nelemans, G., \& Belczynski, K. 2019, MNRAS, 482, 5012

Claret, A. 2007, A\&A, 467, 1389

Crowther, P. A., Schnurr, O., Hirschi, R., et al. 2010, MNRAS, 408, 731

de Mink, S. E., \& Belczynski, K. 2015, ApJ, 814, 58

de Mink, S. E., \& Mandel, I. 2016, MNRAS, 460, 3545

Detmers, R. G., Langer, N., Podsiadlowski, P., \& Izzard, R. G. 2008, A\&A, 484, 831

Di Carlo, U. N., Giacobbo, N., Mapelli, M., et al. 2019, MNRAS, 487, 2947

Dominik, M., Belczynski, K., Fryer, C., et al. 2012, ApJ, 759, 52

Dominik, M., Belczynski, K., Fryer, C., et al. 2013, ApJ, 779, 72

Downing, J. M. B., Benacquista, M. J., Giersz, M., \& Spurzem, R. 2010, MNRAS, 407, 1946

Duchêne, G., \& Kraus, A. 2013, ARA\&A, 51, 269

Eggenberger, P., Meynet, G., Maeder, A., et al. 2008, Ap\&SS, 316, 43

Ekström, S., Georgy, C., Eggenberger, P., et al. 2012, A\&A, 537, A146

Eldridge, J. J., \& Stanway, E. R. 2016, MNRAS, 462, 3302

Farmer, R., Fields, C. E., Petermann, I., et al. 2016, ApJS, 227, 22

Farr, W. M., Stevenson, S., Miller, M. C., et al. 2017, Nature, 548, 426

Farr, B., Holz, D. E., \& Farr, W. M. 2018, ApJ, 854, L9

Faucher-Giguère, C.-A., \& Kaspi, V. M. 2006, ApJ, 643, 332

Fields, C. E., Timmes, F. X., Farmer, R., et al. 2018, ApJS, 234, 19

Fishbach, M., \& Holz, D. E. 2017, ApJ, 851, L25

Fishbach, M., Holz, D. E., \& Farr, B. 2017, ApJ, 840, L24

Foglizzo, T., Guilet, J., \& Sato, J. 2009, in SF2A-2009: Proceedings of the Annual Meeting of the French Society of Astronomy and Astrophysics, eds. M. Heydari-Malayeri, C. Reyl'E, \& R. Samadi, 143

Freytag, B., Ludwig, H. G., \& Steffen, M. 1996, A\&A, 313, 497

Fryer, C. L., \& Heger, A. 2000, ApJ, 541, 1033

Fryer, C. L., \& Kusenko, A. 2006, ApJS, 163, 335

Fryer, C. L., \& Young, P. A. 2007, ApJ, 659, 1438

Fryer, C. L., Woosley, S. E., \& Hartmann, D. H. 1999, ApJ, 526, 152

Fryer, C. L., Woosley, S. E., \& Heger, A. 2001, ApJ, 550, 372

Fryer, C. L., Belczynski, K., Wiktorowicz, G., et al. 2012, ApJ, 749, 91

Fryer, C. L., Lloyd-Ronning, N., Wollaeger, R., et al. 2019, Eur. Phys. J. A, 55 , A132

Fuller, J., \& Ma, L. 2019, ApJ, 881, L1

Fuller, J., Piro, A. L., \& Jermyn, A. S. 2019, MNRAS, 485, 3661

Georgy, C., Ekström, S., Meynet, G., et al. 2012, A\&A, 542, A29

Georgy, C., Ekström, S., Eggenberger, P., et al. 2013, A\&A, 558, A103

Gerosa, D., \& Berti, E. 2017, Phys. Rev. D, 95, 124046
Gerosa, D., Kesden, M., Berti, E., O’Shaughnessy, R., \& Sperhake, U. 2013, Phys. Rev. D, 87, 104028

Gerosa, D., Kesden, M., Sperhake, U., Berti, E., \& O’Shaughnessy, R. 2015, Phys. Rev. D, 92, 064016

Gerosa, D., Berti, E., O'Shaughnessy, R., et al. 2018, Phys. Rev. D, 98, 084036

Giersz, M., Heggie, D. C., Hurley, J. R., \& Hypki, A. 2013, MNRAS, 431, 2184

Glebbeek, E., Gaburov, E., de Mink, S. E., Pols, O. R., \& Portegies Zwart, S. F. 2009, A\&A, 497, 255

Green, A. M. 2017, Phys. Rev. D, 96, 043020

Groh, J. H., Ekström, S., Georgy, C., et al. 2019, A\&A, 627, A24

Gültekin, K., Miller, M. C., \& Hamilton, D. P. 2004, ApJ, 616, 221

Gültekin, K., Miller, M. C., \& Hamilton, D. P. 2006, ApJ, 640, 156

Hainich, R., Oskinova, L. M., Shenar, T., et al. 2018, A\&A, 609, A94

Hamilton, A. J. S., \& Sarazin, G. L. 1982, MNRAS, 198, 59

Heger, A., \& Woosley, S. E. 2002, ApJ, 567, 532

Heger, A., Langer, N., \& Woosley, S. E. 2000, ApJ, 528, 368

Heger, A., Woosley, S. E., \& Spruit, H. C. 2005, ApJ, 626, 350

Herwig, F. 2000, A\&A, 360, 952

Holgado, A. M., Ricker, P. M., \& Huerta, E. A. 2018, ApJ, 857, 38

Hong, J., Vesperini, E., Askar, A., et al. 2018, MNRAS, 480, 5645

Hotokezaka, K., \& Piran, T. 2017, ApJ, 842, 111

Hurley, J. R., Pols, O. R., \& Tout, C. A. 2000, MNRAS, 315, 543

Hurley, J. R., Tout, C. A., \& Pols, O. R. 2002, MNRAS, 329, 897

Hurley, J. R., Sippel, A. C., Tout, C. A., \& Aarseth, S. J. 2016, PASA, 33, e036

Igoshev, A. P., \& Popov, S. B. 2013, MNRAS, 432, 967

Janiuk, A. 2017, ApJ, 837, 39

Jones, S., Hirschi, R., Pignatari, M., et al. 2015, MNRAS, 447, 3115

Kazeroni, R., Guilet, J., \& Foglizzo, T. 2016, MNRAS, 456, 126

Khan, S., Husa, S., Hannam, M., et al. 2016, Phys. Rev. D, 93, 044007

King, A. R. 2004, Nucl. Phys. B Proc. Suppl., 132, 376

King, A. R., \& Ritter, H. 1999, MNRAS, 309, 253

Klencki, J., Moe, M., Gladysz, W., et al. 2018, A\&A, 619, A77

Kroupa, P. 2001, MNRAS, 322, 231

Kroupa, P., Weidner, C., Pflamm-Altenburg, J., et al. 2013, The Stellar and SubStellar Initial Mass Function of Simple and Composite Populations, 115

Kruckow, M. U., Tauris, T. M., Langer, N., Kramer, M., \& Izzard, R. G. 2018, MNRAS, 481, 1908

Kusenko, A., \& Segrè, G. 1996, Phys. Rev. Lett., 77, 4872

Kushnir, D., Zaldarriaga, M., Kollmeier, J. A., \& Waldman, R. 2016, MNRAS, 462, 844

Kushnir, D., Zaldarriaga, M., Kollmeier, J. A., \& Waldman, R. 2017, MNRAS, 467, 2146

Lai, D., \& Qian, Y.-Z. 1998, ApJ, 495, L103

Leung, S.-C., Nomoto, K., \& Blinnikov, S. 2019, ApJ, 887, 72

Limongi, M., \& Chieffi, A. 2018, ApJS, 237, 13

Lipunov, V. M., Postnov, K. A., \& Prokhorov, M. E. 1997, Astron. Lett., 23, 492

LVC 2019a, GCN Circ., 25324

LVC 2019b, GCN Circ., 25333

Ma, L., \& Fuller, J. 2019, MNRAS, 488, 4338

MacLeod, M., \& Ramirez-Ruiz, E. 2015, ApJ, 803, 41

MacLeod, M., Antoni, A., Murguia-Berthier, A., Macias, P., \& Ramirez-Ruiz, E. 2017, ApJ, 838, 56

Madau, P., \& Dickinson, M. 2014, ARA\&A, 52, 415

Madau, P., \& Fragos, T. 2017, ApJ, 840, 39

Maeder, A., \& Meynet, G. 2000, A\&A, 361, 159

Maiolino, R., Nagao, T., Grazian, A., et al. 2008, A\&A, 488, 463

Manchester, R. N., \& Taylor, J. H. 1977, Pulsars (San Francisco: W. H. Freeman)

Mandel, I. 2016, MNRAS, 456, 578

Mandel, I., \& de Mink, S. E. 2016, MNRAS, 458, 2634

Mandel, I., \& Farmer, A. 2018, ArXiv e-prints [arXiv:1806. 05820]

Mandel, I., \& O'Shaughnessy, R. 2010, Classical Quantum Gravity, 27, 114007

Mapelli, M. 2016, MNRAS, 459, 3432

Mapelli, M., Giacobbo, N., Santoliquido, F., \& Artale, M. C. 2019, MNRAS, 487, 2

Marchant, P., Langer, N., Podsiadlowski, P., Tauris, T. M., \& Moriya, T. J. 2016, A\&A, 588, A50

Marchant, P., Renzo, M., Farmer, R., et al. 2019, ApJ, 882, 36

Marks, M., \& Kroupa, P. 2012, A\&A, 543, A8

Marks, M., Kroupa, P., Dabringhausen, J., \& Pawlowski, M. S. 2012, MNRAS, 422, 2246

Meurs, E. J. A., \& van den Heuvel, E. P. J. 1989, A\&A, 226, 88

Meynet, G., \& Maeder, A. 1997, A\&A, 321, 465

Meynet, G., Chomienne, V., Ekström, S., et al. 2015, A\&A, 575, A60

Miller, M. C., \& Hamilton, D. P. 2002a, MNRAS, 330, 232

Miller, M. C., \& Hamilton, D. P. 2002b, ApJ, 576, 894

Miller, M. C., \& Miller, J. M. 2015, Phys. Rep., 548, 1

Moe, M., \& Di Stefano, R. 2013, ApJ, 778, 95

Moe, M., \& Di Stefano, R. 2017, ApJS, 230, 15 
Morawski, J., Giersz, M., Askar, A., \& Belczynski, K. 2018, MNRAS, 481, 2168 Murguia-Berthier, A., MacLeod, M., Ramirez-Ruiz, E., Antoni, A., \& Macias, P. 2017, ApJ, 845, 173

Neijssel, C. J., Vigna-Gómez, A., Stevenson, S., et al. 2019, MNRAS, 490, 3740

Nieuwenhuijzen, H., \& de Jager, C. 1990, A\&A, 231, 134

Noutsos, A., Schnitzeler, D. H. F. M., Keane, E. F., Kramer, M., \& Johnston, S. 2013, MNRAS, 430, 2281

Nugis, T., \& Lamers, H. J. G. L. M. 2000, A\&A, 360, 227

Ohsuga, K. 2007, ApJ, 659, 205

O’Leary, R. M., O’Shaughnessy, R., \& Rasio, F. A. 2007, Phys. Rev. D, 76, 061504

Oskinova, L. M., Todt, H., Ignace, R., et al. 2011, MNRAS, 416, 1456

Oskinova, L. M., Sun, W., Evans, C. J., et al. 2013, ApJ, 765, 73

Packet, W. 1981, A\&A, 102, 17

Pakull, M. W., \& Mirioni, L. 2003, in Revista Mexicana de Astronomia y Astrofisica Conference Series, eds. J. Arthur, \& W. J. Henney, 15, 197

Park, D., Kim, C., Lee, H. M., Bae, Y.-B., \& Belczynski, K. 2017, MNRAS, 469, 4665

Pavlovskii, K., Ivanova, N., Belczynski, K., \& Van, K. X. 2017, MNRAS, 465, 2092

Paxton, B., Bildsten, L., Dotter, A., et al. 2011, ApJS, 192, 3

Paxton, B., Cantiello, M., Arras, P., et al. 2013, ApJS, 208, 4

Paxton, B., Marchant, P., Schwab, J., et al. 2015, ApJS, 220, 15

Paxton, B., Schwab, J., Bauer, E. B., et al. 2018, ApJS, 234, 34

Paxton, B., Smolec, R., Gautschy, A., et al. 2019, ApJS, 243, 10

Perna, R., Wang, Y.-H., Farr, W. M., Leigh, N., \& Cantiello, M. 2019, ApJ, 878, L1

Podsiadlowski, P., Joss, P. C., \& Hsu, J. J. L. 1992, ApJ, 391, 246

Popov, S. B., \& Turolla, R. 2012, Ap\&SS, 341, 457

Portegies Zwart, S. F., \& McMillan, S. L. W. 2000, ApJ, 528, L17

Portegies Zwart, S. F., Baumgardt, H., Hut, P., Makino, J., \& McMillan, S. L. W. 2004, Nature, 428, 724

Qin, Y., Fragos, T., Meynet, G., et al. 2018, A\&A, 616, A28

Qin, Y., Marchant, P., Fragos, T., Meynet, G., \& Kalogera, V. 2019, ApJ, 870 L18

Raghavan, D., McAlister, H. A., Henry, T. J., et al. 2010, ApJS, 190, 1

Ramachandran, V., Hamann, W. R., Oskinova, L. M., et al. 2019, A\&A, 625, A104

Ramírez-Agudelo, O. H., Simón-Díaz, S., Sana, H., et al. 2013, A\&A, 560, A29

Rantsiou, E., Burrows, A., Nordhaus, J., \& Almgren, A. 2011, ApJ, 732, 57

Repetto, S., \& Nelemans, G. 2015, MNRAS, 453, 3341

Repetto, S., Igoshev, A. P., \& Nelemans, G. 2017, MNRAS, 467, 298

Ricker, P. M., \& Taam, R. E. 2008, ApJ, 672, L41

Rodriguez, C. L., Haster, C.-J., Chatterjee, S., Kalogera, V., \& Rasio, F. A. 2016a ApJ, 824, L8

Rodriguez, C. L., Zevin, M., Pankow, C., Kalogera, V., \& Rasio, F. A. 2016b, ApJ, 832, L2

Rodriguez, C. L., Chatterjee, S., \& Rasio, F. A. 2016c, Phys. Rev. D, 93, 084029

Rodriguez, C. L., Amaro-Seoane, P., Chatterjee, S., et al. 2018, Phys. Rev. D, 98 , 123005

Sadowski, A., Belczynski, K., Bulik, T., et al. 2008, ApJ, 676, 1162

Samsing, J. 2018, Phys. Rev. D, 97, 103014

Sana, H., de Mink, S. E., de Koter, A., et al. 2012, Science, 337, 444
Shakura, N. I., \& Sunyaev, R. A. 1973, A\&A, 24, 337

Sądowski, A., Lasota, J.-P., Abramowicz, M. A., \& Narayan, R. 2016, MNRAS, 456, 3915

Socrates, A., Blaes, O., Hungerford, A., \& Fryer, C. L. 2005, ApJ, 632, 531

Spera, M., Mapelli, M., \& Bressan, A. 2015, MNRAS, 451, 4086

Spera, M., Mapelli, M., Giacobbo, N., et al. 2019, MNRAS, 485, 889

Spruit, H. C. 1999, A\&A, 349, 189

Spruit, H. C. 2002, A\&A, 381, 923

Stevenson, S., Vigna-Gómez, A., Mandel, I., et al. 2017, Nat. Commun., 8, 14906

Stevenson, S., Sampson, M., Powell, J., et al. 2019, ApJ, 882, 121

Surman, R., McLaughlin, G. C., \& Hix, W. R. 2006, ApJ, 643, 1057

Tamborra, I., Hanke, F., Janka, H.-T., et al. 2014, ApJ, 792, 96

Tutukov, A. V., \& Yungelson, L. R. 1993, MNRAS, 260, 675

Vagnozzi, S. 2019, Atoms, 7, 41

Valsecchi, F., Glebbeek, E., Farr, W. M., et al. 2010, Nature, 468, 77

Vanbeveren, D., Mennekens, N., Shara, M. M., \& Moffat, A. F. J. 2018, A\&A, 615, A65

VanLandingham, J. H., Miller, M. C., Hamilton, D. P., \& Richardson, D. C. 2016, ApJ, 828, 77

Venumadhav, T., Zackay, B., Roulet, J., Dai, L., \& Zaldarriaga, M. 2019, ArXiv e-prints [arXiv:1904.07214]

Vink, J. S., de Koter, A., \& Lamers, H. J. G. L. M. 2001, A\&A, 369, 574

Vitale, S., Lynch, R., Sturani, R., \& Graff, P. 2017a, Classical Quantum Gravity, 34, 03LT01

Vitale, S., Gerosa, D., Haster, C.-J., Chatziioannou, K., \& Zimmerman, A. 2017b, Phys. Rev. Lett., 119, 251103

Vlahakis, N., \& Königl, A. 2001, ApJ, 563, L129

Voss, R., \& Tauris, T. M. 2003, MNRAS, 342, 1169

Webb, J. J., \& Leigh, N. W. C. 2015, MNRAS, 453, 3278

Wiktorowicz, G., Belczynski, K., \& Maccarone, T. 2014, Binary Systems, their Evolution and Environments, 37

Wiktorowicz, G., Lasota, J.-P., Middleton, M., \& Belczynski, K. 2019, ApJ, 875, 53

Williamson, A. R., Lange, J., O’Shaughnessy, R., et al. 2017, Phys. Rev. D, 96, 124041

Woosley, S. E. 2016, ApJ, 824, L10

Woosley, S. E. 2017, ApJ, 836, 244

Worley, A., Krastev, P. G., \& Li, B.-A. 2008, ApJ, 685, 390

Wyrzykowski, Ł., \& Mandel, I. 2020, A\&A, 363, A20

Wysocki, D., Gerosa, D., O'Shaughnessy, R., et al. 2018, Phys. Rev. D, 97, 043014

Xu, X.-J., \& Li, X.-D. 2010, ApJ, 722, 1985

Zackay, B., Venumadhav, T., Dai, L., Roulet, J., \& Zaldarriaga, M. 2019, Phys. Rev. D, 100, 023007

Zahid, H. J., Dima, G. I., Kudritzki, R.-P., et al. 2014, ApJ, 791, 130

Zahn, J.-P. 1977, A\&A, 57, 383

Zahn, J.-P. 1989, A\&A, 220, 112

Zahn, J.-P. 1992, A\&A, 265, 115

Zaldarriaga, M., Kushnir, D., \& Kollmeier, J. A. 2018, MNRAS, 473, 4174

Zevin, M., Samsing, J., Rodriguez, C., Haster, C.-J., \& Ramirez-Ruiz, E. 2019, ApJ, 871, 91

Ziosi, B. M., Mapelli, M., Branchesi, M., \& Tormen, G. 2014, MNRAS, 441, 3703 


\section{Appendix A: Additional information}

Data availability: data generated during this project with population synthesis code StarTrack is available online ${ }^{10}$ under the tab "Download/2020: Double Compact Objects/Belczynski et al. 2020". Code availability: the StarTrack code is not an open source code and is not publicly available. New models will be calculated upon requests to the first author: chrisbelczynski@ gmail.com.

\section{A.1. Geneva stellar models}

The physics included in the employed Geneva rotating models are described in detail in Eggenberger et al. (2008), Ekström et al. (2012). The models have been computed with a modest core-overshooting during the core $\mathrm{H}$ - and He-burning phase (the core has been extended beyond the Schwarzschild limit by a length given by $10 \%$ of the local pressure scale height). Mass loss rates by stellar winds are accounted for (see the above references for the details). The effects of rotation are included according to the theory by Zahn (1992). In these models, the transport of the angular momentum and the mixing of the chemical species in the radiative zones are due to shear instabilities and meridional currents. In contrast with many stellar models in which the effects of meridional currents are accounted for by a diffusion equation, in the present models the angular momentum transport is accounted for by solving an advective equation. In essence, transport by meridional currents is an advective process. Diffusion treatments can lead not only to wrong estimates of the amplitude, but also to wrong signs (a diffusive process always tends to flatten any gradient, while an advective process can increase gradients in some circumstances). We also note that shear instabilities are the main drivers for the transport of the chemical species, while angular momentum is mainly transported by meridional currents. The angular momentum transport by meridional currents mildly couples the core rotation with that of the envelope. In the convective zones, the transport of angular momentum and chemical species is assumed to be extremely efficient: convective zones are assumed to rotate as a solid body and the chemical composition is homogenized.

In Table A.1, we present a suite of Geneva evolutionary models (Ekström et al. 2012; Georgy et al. 2013; Groh et al. 2019) and Eggenberger et al. (in prep.) with $M_{\text {zams }}=9-120 M_{\odot}$ for a wide range of chemical compositions: $Z=0.014-0.0004$. Specifically, we are listing CO core mass for each evolutionary model along with our estimate of the associated BH spin magnitude (see Fig. 1 for a plot of the relation between the two).

The spin magnitude is very sensitive to the $\mathrm{BH}$ mass $\left(\propto 1 / M_{\mathrm{BH}}^{2}\right.$; see Eq. (2)). The $\mathrm{CO}$ core mass may be taken as a proxy for BH mass (cf. Appendix A.3 and Fig. A.2). Below we present the behavior of the $\mathrm{CO}$ core mass within the Geneva models. This discussion explains the non-monotonic dependence of BH spin on metallicity.

As naturally expected, the $\mathrm{CO}$ core mass increases with the initial stellar mass within models of the same metallicity. This intuitive trend is reversed only at the highest metallicity considered here $(Z=0.014)$, at which stellar winds are most efficient in mass removal. In particular, this is true for very massive stars $\left(M_{\text {zams }} \gtrsim 100 M_{\odot}\right)$.

Another intuitive expectation is not supported by these stellar models. For a given initial stellar mass, one may naively expect that the CO core mass would increase with decreasing metallicity, as winds are getting weaker and star remains more massive.

${ }_{10}$ wwW. syntheticuniverse.org
However, for example for $M_{\mathrm{zams}}=85 M_{\odot}$ we find $M_{\mathrm{CO}, 2}=26.4$, $35.8,27.4,44.2 M_{\odot}$ for $Z=0.014,0.006,0.002,0.0004$, respectively. In the following, we explain this non-monotonic behavior in terms of our adopted stellar evolution model.

Stellar winds are weaker at low metallicity thus this leads to increase of the $\mathrm{CO}$ core mass with decreasing metallicity. This general trend is mitigated by two other physical processes. Decreasing metallicity leads to the formation of extended convective H-burning shells that tend to slow down the growth of the He core (and subsequently the CO core) mass. At a high $Z$, massive stars lose most of their envelopes, so there is no vertical structure available for extended convective $\mathrm{H}$-burning shell. The H-burning shell is compact and moves outwards (once $\mathrm{H}$ is totally depleted) through the envelope, adding mass to the underlying $\mathrm{He}$ core. At a low $Z$, massive stars not only retain their envelopes longer, but they are also more compact, and increased density helps to form extended convective zones. Within the extended convective shell reaching far above H-burning (which occurs only at the shell base), intensive mixing keeps bringing new fuel to the H-burning zone (keeping it in the same position), and keeps redistributing newly formed $\mathrm{He}$ across this large shell. Instead of adding He to the core, the newly formed $\mathrm{He}$ is redistributed through the material of the extended convective shell. Another process that leads to $\mathrm{CO}$ core mass decrease with metallicity is connected to the diffusion of elements due to the action of meridional currents and turbulence. With decreasing metallicity stars are more compact and the vertical scale of diffusion decreases, leading to less effective mixing of fresh fuel into burning zones. This, in turn, lowers the CO core mass.

For the particular set of assumptions used in the Geneva code, the non-monotonic behavior of the $\mathrm{CO}$ core mass is the effect of the complex and metallicity-dependent interplay of strength of stellar winds, the H-burning shell extent, and the model for the efficiency of element diffusion within the meridional current. This explains the non-monotonic metallicity dependence of our BH natal spin model (see Fig. 1).

\section{A.2. MESA stellar models}

The MESA stellar models were evolved using MESA revision 10398 (Paxton et al. 2011, 2013, 2015, 2018, 2019). The models are evolved from the pre-main sequence to core He-depletion. We use temporal and spatial parameters similar to those used in Farmer et al. (2016) and Fields et al. (2018) that provide convergence to the $\approx 10 \%$ level. The MESA models use the mesa-49. net network that follows 49 isotopes from ${ }^{1} \mathrm{H}$ to ${ }^{34} \mathrm{~S}$. We include mass loss using the "Dutch" wind scheme with an efficiency value of 0.8 (Nieuwenhuijzen \& de Jager 1990; Nugis \& Lamers 2000; Vink et al. 2001; Glebbeek et al. 2009). We use the Ledoux criterion for convection with an efficiency parameter of $\alpha_{\mathrm{MLT}}=1.6$, and the mlt++ approximation for convection (Paxton et al. 2013).

Additional mixing processes due to convective boundary mixing are included using the exponentially decaying diffusion coefficient framework of Herwig (2000) based on hydrodynamic simulations of Freytag et al. (1996). The following values of $f$ were used: $f=0.014$ above $\mathrm{H}$ - and He-burning regions, $f=0.001$ below $\mathrm{H}$ - and He-burning regions, $f=0$ elsewhere. We note that the additional parameter, $f_{0}=0.001$ was used, where $f_{0} H_{\mathrm{P}}$ is the distance from the boundary inside the convective zone where the exponential decay starts. Jones et al. (2015) compare GENEC models to MESA models and find that using $f=0.022$ on top of convective core $\mathrm{H}$ and He burning 
Table A.1. Geneva stellar models.

\begin{tabular}{|c|c|c|c|c|c|c|c|c|c|c|}
\hline$Z$ & $\begin{array}{r}M_{\mathrm{ZAMS}} \\
{\left[M_{\odot}\right]} \\
\end{array}$ & $\begin{array}{c}V_{\text {rot, }} \\
{\left[\mathrm{km} \mathrm{s}^{-1}\right]}\end{array}$ & $\begin{array}{c}M_{\mathrm{He}} \\
{\left[M_{\odot}\right]}\end{array}$ & $\begin{array}{c}M_{\mathrm{CO}, 1} \\
{\left[M_{\odot}\right]} \\
\end{array}$ & $\begin{array}{r}M_{\mathrm{CO}, 2} \\
{\left[M_{\odot}\right]} \\
\end{array}$ & $\begin{array}{c}P_{\mathrm{NS}}, a_{\text {spin }} \\
{[\mathrm{ms}]}\end{array}$ & $\begin{array}{c}M_{\mathrm{NS} / \mathrm{BH}} \\
{\left[M_{\odot}\right]}\end{array}$ & $\begin{array}{c}M_{\text {star, },} \\
{\left[M_{\odot}\right]} \\
\end{array}$ & $\begin{array}{c}M_{\text {star,st } 1} \\
{\left[M_{\odot}\right]}\end{array}$ & $\begin{array}{c}M_{\text {star, st2 }} \\
{\left[M_{\odot}\right]} \\
\end{array}$ \\
\hline \multirow[t]{10}{*}{$Z=0.014$} & 9 & 248 & 2.9 & 1.61 & 2.40 & 0.6 & 1.18 & 8.5 & 8.5 & 8.9 \\
\hline & 12 & 262 & 3.8 & 2.26 & 3.40 & 0.6 & 1.19 & 10.2 & 11.0 & 11.7 \\
\hline & 15 & 271 & 5.1 & 3.07 & 4.59 & 0.5 & 1.35 & 11.1 & 11.3 & 13.9 \\
\hline & 20 & 274 & 7.1 & 4.50 & 6.86 & 0.5 & 1.76 & 7.2 & 12.3 & 17.6 \\
\hline & 25 & 295 & 9.7 & 6.69 & 9.38 & 0.90 & 9.66 & 9.7 & 9.2 & 20.4 \\
\hline & 32 & 306 & 10.1 & 7.45 & 10.1 & 0.88 & 6.24 & 10.1 & 10.5 & 23.4 \\
\hline & 40 & 314 & 12.3 & 9.29 & 12.3 & 0.87 & 7.88 & 12.3 & 11.6 & 22.1 \\
\hline & 60 & 346 & 18.0 & 14.2 & 18.0 & 0.62 & 14.2 & 18.0 & 13.5 & 36.0 \\
\hline & 85 & 368 & 26.4 & 21.6 & 26.4 & 0.29 & 21.6 & 26.4 & 18.1 & 52.6 \\
\hline & 120 & 389 & 19.0 & 15.2 & 19.0 & 0.13 & 15.2 & 19.0 & 20.7 & 66.9 \\
\hline \multirow[t]{8}{*}{$Z=0.006$} & 15 & 271 & 5.1 & 2.87 & 4.71 & 0.5 & 1.30 & 14.0 & 13.5 & 14.5 \\
\hline & 20 & 292 & 7.2 & 4.41 & 7.02 & 0.5 & 1.72 & 13.9 & 16.7 & 19.0 \\
\hline & 25 & 301 & 9.6 & 6.50 & 9.55 & 0.80 & 9.62 & 10.5 & 17.8 & 22.8 \\
\hline & 32 & 334 & 13.5 & 9.98 & 13.4 & 0.90 & 11.6 & 13.6 & 17.5 & 27.5 \\
\hline & 40 & 334 & 18.9 & 14.9 & 18.7 & 0.90 & 18.9 & 18.9 & 14.8 & 21.3 \\
\hline & 60 & 378 & 32.8 & 28.3 & 32.8 & 0.90 & 28.2 & 32.8 & 18.9 & 38.6 \\
\hline & 85 & 410 & 35.8 & 30.2 & 35.8 & 0.37 & 30.1 & 35.8 & 28.8 & 61.5 \\
\hline & 120 & 435 & 52.5 & 45.1 & 52.4 & 0.25 & 42.3 & 52.5 & 31.4 & 77.9 \\
\hline \multirow[t]{10}{*}{$Z=0.002$} & 9 & 255 & 2.8 & 1.55 & 1.59 & 0.5 & 1.20 & 8.9 & 8.4 & 8.9 \\
\hline & 12 & 271 & 3.9 & 2.01 & 3.17 & 0.5 & 1.20 & 11.8 & 9.4 & 11.3 \\
\hline & 15 & 303 & 5.0 & 2.76 & 4.65 & 0.5 & 1.26 & 14.7 & 12.8 & 14.6 \\
\hline & 20 & 305 & 7.2 & 4.36 & 7.04 & 0.5 & 1.73 & 18.7 & 17.8 & 19.3 \\
\hline & 25 & 319 & 9.6 & 6.29 & 9.42 & 0.90 & 9.56 & 21.8 & 19.8 & 23.4 \\
\hline & 32 & 338 & 13.1 & 9.32 & 13.0 & 0.85 & 10.2 & 24.1 & 18.9 & 28.0 \\
\hline & 40 & 358 & 17.6 & 13.3 & 17.5 & 0.90 & 17.6 & 27.4 & 14.5 & 17.1 \\
\hline & 60 & 400 & 31.6 & 26.7 & 27.4 & 0.001 & 31.6 & 39.1 & 22.8 & 39.3 \\
\hline & 85 & 435 & 45.3 & 26.7 & 27.4 & 0.28 & 43.0 & 74.8 & 35.7 & 66.6 \\
\hline & 120 & 438 & 85.6 & 76.4 & 83.2 & PSN & 0.0 & 85.6 & 46.6 & 91.6 \\
\hline \multirow[t]{10}{*}{$Z=0.0004$} & 9 & 270 & 1.6 & 1.54 & 1.59 & 0.6 & 1.20 & 8.90 & 8.9 & 9.0 \\
\hline & 12 & 285 & 3.9 & 2.02 & 3.28 & 0.6 & 1.19 & 11.9 & 11.8 & 11.9 \\
\hline & 15 & 319 & 5.0 & 2.87 & 4.81 & 0.5 & 1.28 & 14.9 & 14.8 & 14.9 \\
\hline & 20 & 314 & 7.8 & 4.84 & 7.35 & 0.4 & 1.86 & 19.4 & 19.7 & 19.9 \\
\hline & 25 & 343 & 10.0 & 6.60 & 9.81 & 0.88 & 10.0 & 24.0 & 24.4 & 24.8 \\
\hline & 32 & 366 & 12.6 & 8.71 & 12.5 & 0.80 & 8.86 & 30.5 & 30.0 & 31.4 \\
\hline & 40 & 393 & 16.9 & 12.5 & 16.9 & 0.82 & 12.5 & 34.6 & 15.6 & 23.4 \\
\hline & 60 & 435 & 28.4 & 28.4 & 27.8 & 0.79 & 28.4 & 49.9 & 24.9 & 41.7 \\
\hline & 85 & 469 & 47.0 & 40.1 & 44.2 & 0.26 & 44.6 & 57.8 & 37.9 & 68.5 \\
\hline & 120 & 463 & 70.2 & 62.1 & 65.3 & PSN & 0.0 & 92.5 & 66.6 & 104 \\
\hline
\end{tabular}

Notes. For all models we list: $Z$ : metallicity, $M_{\mathrm{ZAMS}}$ : initial star mass, $V_{\mathrm{rot}, \mathrm{i}}$ : initial rotation at equator, $M_{\mathrm{He}}$ : He core mass, $M_{\mathrm{CO}, 1}$ : CO core mass defined by $<1 \% \mathrm{He}$ (used in MESA), $M_{\mathrm{CO}, 2}$ : CO core mass defined by $>20 \% \mathrm{CO}$ (adopted in our study), $P_{\mathrm{NS}}, a_{\text {spin }}$ : for NSs, the NS spin period in milliseconds, for BHs, the dimensionless spin magnitude, $M_{\mathrm{NS} / \mathrm{BH}}$ : remnant mass, $M_{\mathrm{star}, \mathrm{g}}$ : final mass of a star in Geneva code, $M_{\mathrm{star}, \mathrm{st}}$ : final mass of a star in StarTrack with standard winds, $M_{\text {star,st2 }}$ : final mass of a star in StarTrack with reduced (30\%) winds.

in MESA matches GENEC models with a penetrative overshoot, $\alpha_{\mathrm{ov}}=0.2 H_{\mathrm{P}}$. Given that the GENEC models used in this paper use $\alpha_{\mathrm{ov}}=0.1 H_{\mathrm{P}}$, using $f=0.014$ in MESA yields similar core masses and lifetimes.

In MESA, rotation is implemented using the shellular approximation (Zahn 1992; Meynet \& Maeder 1997). In our models, we initialize solid body, uniform rotation at the zero age mainsequence as a fraction of the Keplerian critical rotation rate, in most of our models this value is $40 \%$. We use the suggested values from (Heger et al. 2000) for the diffusion coefficients for the transport of angular momentum and material due to various instabilities. Among the most influential of these mechanisms included in the MESA models, there is the Tayler-Spruit dynamo. The modeling of this dynamo in the stellar models increases the efficiency in angular momentum transport and can lead to a significantly different angular momentum profiles and core rotation rates (Heger et al. 2005, see also Sect. 4.1).

We find that the Taylor-Spruit dynamo essentially dominates the transport of angular momentum in our MESA models and is very efficient at transporting the angular momentum from the core to the upper layers, from which it is lost in winds. As a result, all our models end with a very similar (small) amount of angular momentum and thus small spin parameter $a<0.15$; see Fig. 2 and also Table A.2. Given that stronger stellar winds lead to a more efficient loss of angular momentum, one would expect a dependence of the final spin on mass and metallicity. We find a hint of such a dependence in our models: the more massive as well as the higher metallicity models tend to end with systematically lower final spins (Fig. 2). However, this is not very clear in our models and the impact of mass and 
Table A.2. MESA stellar models with $40 \%$ critical initial rotation.

\begin{tabular}{|c|c|c|c|c|c|c|c|c|c|c|}
\hline$Z$ & $\begin{array}{r}M_{\text {ZAMS }} \\
{\left[M_{\odot}\right]} \\
\end{array}$ & $\begin{array}{c}V_{\text {rot, },} \\
{\left[\mathrm{km} \mathrm{s}^{-1}\right]}\end{array}$ & $\begin{array}{c}M_{\mathrm{He}} \\
{\left[M_{\odot}\right]}\end{array}$ & $\begin{array}{r}M_{\mathrm{CO}, 1} \\
{\left[M_{\odot}\right]} \\
\end{array}$ & $\begin{array}{r}M_{\mathrm{CO}, 2} \\
{\left[M_{\odot}\right]} \\
\end{array}$ & $\begin{array}{c}P_{\mathrm{NS}}, a_{\text {spin }} \\
{[\mathrm{ms}]}\end{array}$ & $\begin{array}{r}M_{\mathrm{NS} / \mathrm{BH}} \\
{\left[M_{\odot}\right]}\end{array}$ & $\begin{array}{r}M_{\text {star,m }} \\
{\left[M_{\odot}\right]} \\
\end{array}$ & $\begin{array}{r}M_{\text {star,st1 }} \\
{\left[M_{\odot}\right]} \\
\end{array}$ & $\begin{array}{r}M_{\text {star,st2 }} \\
{\left[M_{\odot}\right]} \\
\end{array}$ \\
\hline \multirow[t]{9}{*}{$Z=0.014$} & 10 & 257 & 2.9 & 1.2 & 1.5 & 8.0 & 1.20 & 3.4 & 9.4 & 9.8 \\
\hline & 15 & 270 & 4.5 & 2.2 & 2.7 & 6.4 & 1.27 & 5.7 & 11.3 & 13.9 \\
\hline & 20 & 274 & 6.7 & 3.6 & 4.6 & 4.4 & 1.79 & 10.8 & 12.3 & 17.6 \\
\hline & 25 & 276 & 7.8 & 4.2 & 6.2 & 0.094 & 6.16 & 19.9 & 9.2 & 20.4 \\
\hline & 32 & 272 & 12.0 & 7.9 & 9.1 & 0.107 & 9.23 & 25.3 & 10.5 & 23.4 \\
\hline & 40 & 267 & 16.6 & 11.8 & 13.2 & 0.105 & 16.8 & 27.8 & 11.6 & 22.1 \\
\hline & 60 & 254 & 27.6 & 21.1 & 22.6 & 0.083 & 28.0 & 46.5 & 13.5 & 36.0 \\
\hline & 85 & 239 & 41.6 & 33.4 & 35.2 & 0.046 & 42.6 & 56.2 & 18.1 & 52.6 \\
\hline & 120 & 220 & 60.8 & 49.9 & 52.8 & 0.037 & 55.0 & 80.0 & 20.7 & 66.9 \\
\hline \multirow[t]{9}{*}{$Z=0.006$} & 10 & 268 & 2.9 & 1.0 & 1.6 & 6.7 & 1.20 & 3.9 & 9.6 & 9.9 \\
\hline & 15 & 279 & 4.7 & 2.2 & 2.9 & 5.9 & 1.32 & 10.0 & 13.5 & 14.5 \\
\hline & 20 & 293 & 6.8 & 3.8 & 4.6 & 9.7 & 1.79 & 18.3 & 16.7 & 19.0 \\
\hline & 25 & 296 & 8.6 & 5.1 & 6.0 & 0.101 & 8.67 & 23.0 & 17.8 & 22.8 \\
\hline & 32 & 295 & 11.8 & 7.9 & 8.9 & 0.100 & 8.87 & 29.3 & 17.5 & 27.5 \\
\hline & 40 & 289 & 14.4 & 8.9 & 10.3 & 0.099 & 16.1 & 31.3 & 14.8 & 21.3 \\
\hline & 60 & 277 & 26.6 & 20.3 & 21.9 & 0.093 & 26.8 & 52.4 & 18.9 & 38.6 \\
\hline & 85 & 266 & 39.9 & 31.2 & 32.9 & 0.081 & 47.1 & 69.0 & 28.8 & 61.5 \\
\hline & 120 & 251 & 61.8 & 50.6 & 53.3 & 0.072 & 55.0 & 91.7 & 31.4 & 77.9 \\
\hline \multirow[t]{9}{*}{$Z=0.002$} & 10 & 285 & 3.0 & 1.1 & 1.6 & 7.1 & 1.20 & 6.5 & 8.4 & 9.6 \\
\hline & 15 & 298 & 4.9 & 2.5 & 3.0 & 7.7 & 1.35 & 14.7 & 12.8 & 14.6 \\
\hline & 20 & 305 & 6.6 & 3.7 & 4.3 & 7.6 & 1.72 & 19.5 & 17.8 & 19.3 \\
\hline & 25 & 310 & 8.2 & 4.9 & 7.7 & 0.096 & 8.29 & 23.9 & 19.8 & 23.4 \\
\hline & 32 & 312 & 12.0 & 8.1 & 9.0 & 0.104 & 9.11 & 30.4 & 18.9 & 28.0 \\
\hline & 40 & 313 & 15.4 & 10.9 & 12.1 & 0.142 & 15.4 & 37.3 & 14.5 & 17.1 \\
\hline & 60 & 304 & 26.9 & 20.8 & 22.4 & 0.108 & 27.0 & 55.1 & 22.8 & 39.3 \\
\hline & 85 & 286 & 39.5 & 31.6 & 33.6 & 0.090 & 39.7 & 76.9 & 35.7 & 66.6 \\
\hline & 120 & 268 & 60.9 & 50.1 & 54.1 & 0.065 & 55.0 & 105 & 46.6 & 91.6 \\
\hline \multirow[t]{9}{*}{$Z=0.0004$} & 10 & 302 & 3.1 & 1.4 & 1.7 & 7.1 & 1.20 & 9.8 & 9.9 & 10.0 \\
\hline & 15 & 315 & 4.9 & 2.5 & 3.1 & 8.5 & 1.36 & 14.9 & 14.8 & 14.9 \\
\hline & 20 & 323 & 6.8 & 3.9 & 4.6 & 5.2 & 1.79 & 19.7 & 19.7 & 19.9 \\
\hline & 25 & 329 & 8.7 & 5.4 & 6.2 & 0.138 & 8.81 & 24.5 & 24.4 & 24.8 \\
\hline & 32 & 335 & 12.4 & 8.3 & 9.3 & 0.110 & 9.83 & 31.0 & 30.0 & 31.4 \\
\hline & 40 & 338 & 14.3 & 9.7 & 10.7 & 0.109 & 13.7 & 38.3 & 15.6 & 23.4 \\
\hline & 60 & 341 & 40.0 & 32.3 & 34.1 & 0.058 & 43.1 & 55.7 & 24.9 & 41.7 \\
\hline & 85 & 340 & 43.3 & 34.7 & 36.9 & 0.097 & 41.7 & 79.9 & 37.9 & 68.5 \\
\hline & 120 & 334 & 63.1 & 47.3 & 61.1 & 0.072 & 28.4 & 112 & 66.6 & 104 \\
\hline
\end{tabular}

Notes. For all models we list: $Z$ : metallicity, $M_{\mathrm{ZAMS}}$ : initial star mass, $V_{\mathrm{rot}, \mathrm{i}}$ : initial rotation at equator, $M_{\mathrm{He}}$ : He core mass, $M_{\mathrm{CO}, 1}$ : CO core mass defined by $<1 \% \mathrm{He}$ (used in MESA), $M_{\mathrm{CO}, 2}$ : CO core mass defined by $>20 \% \mathrm{CO}$ (adopted in our study), $P_{\mathrm{NS}}, a_{\mathrm{spin}}$ : for NSs, the NS spin period in milliseconds, for BHs, the dimensionless spin magnitude, $M_{\mathrm{NS} / \mathrm{BH}}$ : remnant mass, $M_{\mathrm{star}, \mathrm{m}}$ : final mass of a star in MESA, $M_{\mathrm{star}, \mathrm{st} 1}$ : final mass of a star in StarTrack with standard winds, $M_{\text {star,st2 }}$ : final mass of a star in StarTrack with reduced (30\%) winds.

metallicity is sub-dominant to the fact that the efficient transport of angular momentum through the Taylor-Spruit dynamo leads to small spins.

A star might spin up during its main-sequence evolution due to mass accretion from a companion or be born as a very fast rotator. We mimic this scenario with a single, very fast rotating MESA model ( $80 \%$ of critical rotation) and compare it to the slow and fast-rotating $32 M_{\odot}$ MESA models plotted in Fig. 23 for both magnetic and non-magnetic angular momentum transport. The specific angular momentum profile at core helium depletion of the very fast rotating models is presented in Fig. A.1. It clearly shows, as pointed out earlier in this paper, that the rotation rate at core helium depletion depends mainly on the angular momentum transport mechanism (the difference between the models using the Taylor-Spruit dynamo will be further reduced during the advanced phases of stellar evolution) and not the initial rotation rate. This is a result of the evolution after the main-sequence where the Taylor-Spruit dynamo extracts most of the angular momentum from the core. The core contracts due to the missing energy generation and the envelope expands as a consequence of the virial theorem and energy conservation. The contracting core spins up faster in the models with faster initial rotation, creating a stronger shear between core and envelope. The stronger shear leads to a stronger angular momentum transport of the magnetic dynamo $\left(v_{\mathrm{ST}} \sim-\left(\frac{\mathrm{d} \ln \Omega}{\mathrm{d} \ln r}\right)^{2}\right)$ in a region where stratification is dominated by the chemical composition gradient (Spruit 2002), which is the case for the region above the helium core where the shear develops after the MS evolution in a massive star, resulting in similar specific angular momentum profiles. Therefore, a star that is either spun up by a binary companion or is born fast will still end its life with a slow-spinning core when an efficient angular momentum transport mechanism is active. 


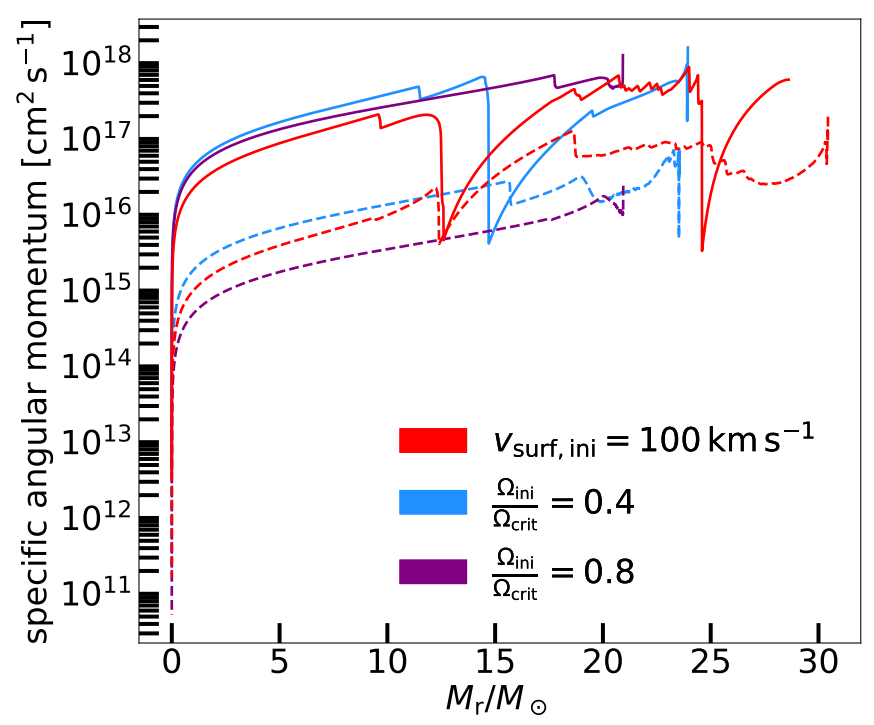

Fig. A.1. Specific angular momentum profile at the end of core helium burning for the $32 M_{\odot}$ MESA models at $Z=0.002$. The models were calculated with different initial rotation rates; slow $\left(100 \mathrm{~km} \mathrm{~s}^{-1}\right.$, red), fast ( $40 \%$ of critical rotation, blue) and very fast ( $80 \%$ of critical rotation, purple). Magnetic (dashed line) and non-magnetic models (solid line) are considered. Large dips in the curve indicate the base of a convective region, where angular momentum transport is very efficient. The maximal extent of the curves to the right indicate the mass of the star. We can see from these curves that the faster rotating models lose more mass in winds due to rotation-enhanced mass loss.

In the non-magnetic models presented in Fig. A.1, most of the angular momentum is transported in the short phase between core hydrogen and helium burning (as in the magnetic models) but the transport is much less efficient. Therefore, the core of models excluding magnetic fields will not be able to slow down and will end up having a high final rotation rate.

There are dips in the angular momentum profiles in Fig. A.1, one of the red curves (slowest rotation) has two dips because when helium is exhausted in the core there is a convective hydrogen zone (starting at $12 M_{\odot}$ ) and the surface convective zone (starting at $25 M_{\odot}$ ) present. This is not the case in the other models because (i) they evolve more to the blue (to higher temperature); (ii) faster rotating models tend to smooth temperature and chemical composition, leading to a shorter time with convective hydrogen core; and (iii) some models lose nearly their entire envelope, hence, there is no surface nor hydrogen convective zone. Models with high rotation end evolution with lower total mass as visible in Fig. A.1. Rotation enhances mass loss in general in several ways: directly by reducing the effective gravity, indirectly by rotation-induced mixing leading to increased luminosities and sometimes leading to quicker evolution to the red supergiant or WR phases. For the very fast-rotating models evolving quasi-chemically homogeneously, the dominant effects are rotation-induced mixing leading to increased luminosities which, in turn, enhances the wind mass loss and reaching the WR phase earlier in the evolution.

We note that apart from the spin values, there are also differences between the final CO core masses between GENEC and MESA models (Tables A.1 and A.2). Some level of discrepancy is not unexpected solely due to the fact that those sets of models were run with slight differences in the efficiency of chemical mixing, criteria for convection, and treatment of convective boundary regions. More importantly, even though both sets of modes were computed using similar prescriptions for mass loss,

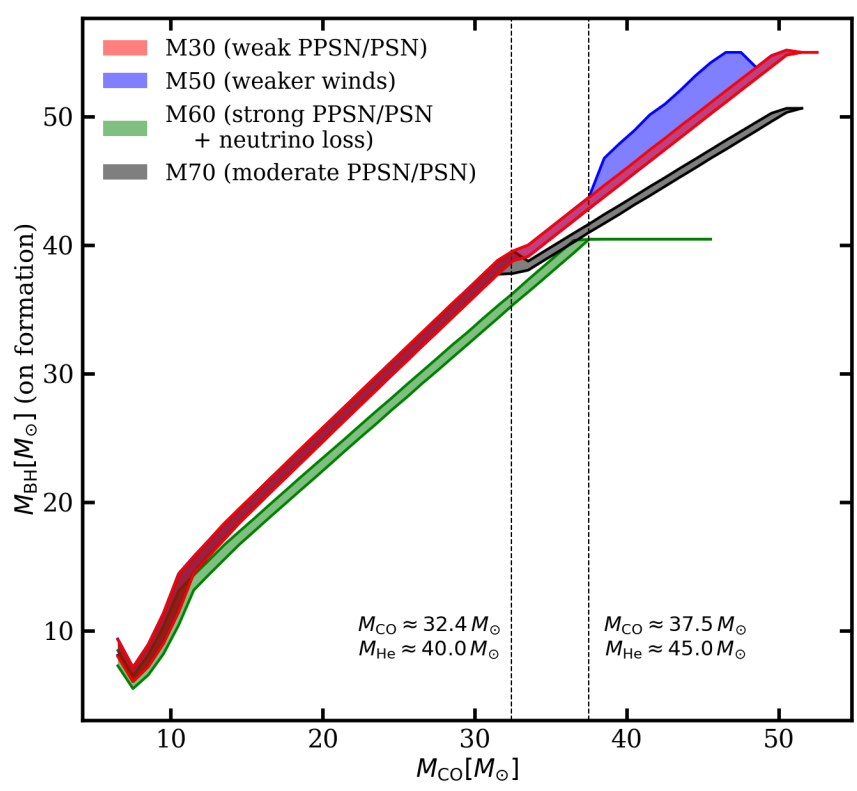

Fig. A.2. Final $\mathrm{CO}$ core masses of $\mathrm{BH}$ progenitors and their corresponding $\mathrm{BH}$ formation masses for the components (both primary and secondary) of detectable merging $\mathrm{BH}-\mathrm{BH}$ systems in four of our models: M30, M50, M60, and M70 (see Table 2 for an overview of all the model ingredients). For readability, the filled areas encapsulate $96 \%$ of all the BHs, excluding few outliers and showcasing the $M_{\mathrm{CO}}$ to $M_{\mathrm{BH}}$ relation for the representative majority. The presented models are different in ways that affect the BH formation masses, that is, the PPSN/PSN prescription (see Sect. 2.3) as well as the assumed fraction of mass lost in neutrinos during a $\mathrm{BH}$ formation (1\% in M30, M50, M70, and $10 \%$ in M60). We note that model M70 only differs from M30 and M50 for $M_{\mathrm{CO}} \gtrsim 32.4 M_{\odot}$, whereas model M50 only differs from M30 for $M_{\mathrm{CO}}>37.5 M_{\odot}$. Those threshold core masses correspond to the threshold helium core masses $M_{\mathrm{He}}$ in different models PPSN/PSN prescriptions.

differences in evolution in the HR diagram alone can lead to significant differences in the total amount of mass lost in winds and therefore the final $\mathrm{CO}$ core masses. This can be most clearly seen when comparing the most massive Solar metallicity models $\left(Z=0.014\right.$, initial masses 60,85 , and $\left.125 M_{\odot}\right)$, in which case the MESA models end their evolution with noticeably higher $\mathrm{CO}$ core masses. We caution that in the case of such massive stars, and especially at higher metallicity, the difficulties in carrying out a numerical treatment of radiation-dominated, superadiabatic envelope layers can lead to significant differences in the HR diagram evolution between different codes (Paxton et al. 2013). Given that such envelopes are likely dynamically unstable and highly turbulent, any 1D models of late evolution of massive stars should be consider highly uncertain.

All MESA inlists used to produce these models are publicly available ${ }^{11}$

\section{A.3. CO core mass versus $\mathrm{BH}$ mass}

The relation between the $\mathrm{BH}$ mass at formation and the final $\mathrm{CO}$ core mass of its progenitor in our simulations is set by formulae based on supernova modeling (the "Rapid" engine of Fryer et al. 2012), together with the pre-supernova mass of the star that is set by stellar and binary evolution. In Fig. A.2, we show the $M_{\mathrm{CO}}-M_{\mathrm{BH}}$ relation for $\mathrm{BH}-\mathrm{BH}$ mergers detectable in the $\mathrm{O} 1 / \mathrm{O} 2$ LIGO runs within models M30, M50, M60, and M70. The

11 mesastar.org 
presented models are different in ways that affect the $\mathrm{BH}$ formation masses, that is, the PPSN/PSN prescription (see Sect. 2.3) as well as the assumed fraction of mass lost in neutrinos during a BH formation (1\% in M30, M50, M70, and 10\% in M60). We note that even though we plot $M_{\mathrm{BH}}$ at the moment of BH formation, it is very similar to the final $\mathrm{BH}$ mass. We also note that the $\mathrm{CO}$ core masses presented in Fig. A.2 are the final $\mathrm{CO}$ core masses (just before the $\mathrm{BH}$ formation), which in some cases have already been reduced due to PPSN mass loss. Those are the $M_{\mathrm{CO}}$ masses that we use in order to assign the newly formed BHs with natal spins (see Sect. 2.1).

In general, the $M_{\mathrm{CO}}$ to $M_{\mathrm{BH}}$ relation is quite similar between our models and, for the most part, almost linear. The initial decrease in $M_{\mathrm{BH}}$ (for $M_{\mathrm{CO}}<7.5 M_{\odot}$ ) and the subsequent increase, followed by a change in slope at around $M_{\mathrm{CO}}=11 M_{\odot}$, are the result of changes in the fraction of material $f_{\mathrm{fb}}$ that falls back onto the proto-NS in the "Rapid" supernovae engine (see Eq. (16) in Fryer et al. 2012). The maximum fall-back $\left(f_{\mathrm{fb}}=1.0\right.$, a direct collapse formation of a $\mathrm{BH}$ ) is expected for the $\mathrm{CO}$ core masses $M_{\mathrm{CO}}$ either in range between 6 and $7 M_{\odot}$ or for $M_{\mathrm{CO}}>11 M_{\odot}$. Partial fall-back and the ejection of some of the pre-SN mass for progenitors with $M_{\mathrm{CO}}$ within 7 and $11 M_{\odot}$ is what is responsible for the dip in $M_{\mathrm{BH}}$ in Fig. A.2 at the lower $M_{\mathrm{CO}}$ end.

The fact that $\mathrm{BH}$ masses are systematically smaller in model M60 compared to other models is a direct consequence of a higher mass fraction being lost in neutrinos during a $\mathrm{BH}$ formation (10\% in M60 compared to $1 \%$ in other models).

The influence of PPSN/PSN kicks in above $M_{\mathrm{CO}} \approx 32.4 M_{\odot}$ for models M30, M50, M70 (which corresponds to $M_{\mathrm{He}} \approx$ $\left.40 M_{\odot}\right)$, and above $M_{\mathrm{CO}} \approx 37.5 M_{\odot}$ in the case of M60 $\left(M_{\mathrm{He}} \approx\right.$ $\left.45 M_{\odot}\right)$. Above this core mass, PPSN removes outer part of the envelope and, therefore, reduces the final pre-SN mass with respect to evolution without PPSN (see Fig. 3). This leads to a smaller mass of the BH formed in direct collapse of the remaining post-PPSN star. As expected, Fig. A.2 reveals that the more mass is lost in PPSN (depending on the model) the smaller the final BH mass. Finally, in the case of most massive stars $\left(M_{\mathrm{He}}>65 M_{\odot}\right)$, all the models assume that a PSN disrupts the entire star and that no remnant remains.

The impact of weaker stellar winds in model M50 (30\% of mass loss in winds at any evolutionary stage compared to other models) on the $M_{\mathrm{CO}}$ to $M_{\mathrm{BH}}$ relation is relatively small, only differentiating models M30 and M50 in any way for $M_{\mathrm{CO}}>40 M_{\odot}$. This is because in most cases of the BH-BH merger formation, both the primary and secondary components are going to lose their entire envelope prior to core collapse anyway, due to RLOF. In fact, as many as $90 \%$ of all the $\mathrm{BH}$ formed from $M_{\mathrm{CO}}>40 M_{\odot}$ progenitors in model M50 fall into the same area in Fig. A.2 as the BHs in model M30.

\section{A.4. Making GW170104}

Figure 8 shows the formation and evolution of a binary which results in a $\mathrm{BH}-\mathrm{BH}$ merger with properties similar to GW170104. This particular system was evolved within our model M20 (see Sect. 2.7 for an overview of our models and Appendix A.7 for an overview of our binary evolution calculations) under the assumptions of Geneva-based BH natal spins (Fig. 1) and no WR spin-up during the BH-WR stage (Sect. 2.5. The progenitor binary was formed in a low-metallicity environment $Z=0.001\left(5 \% Z_{\odot}\right)$ as a pair of MS stars with masses of 94.6 $M_{\odot}$ and $62.5 M_{\odot}$ on a wide orbit, with pericenter distance of $\sim 2000 R_{\odot}$. As the primary ends its MS evolution and rapidly expands as a $\mathrm{HG}$ star, it initiates a stable case-B mass transfer. In model M20, we assume that $20 \%$ of the mass is accreted by the MS companion, while the other $80 \%$ is lost from the system. During mass transfer the primary is stripped of almost all of the H-rich envelope and it becomes a naked helium WR star with mass of $41.7 M_{\odot}$. Only $0.3 \mathrm{Myr}$ later it finishes its nuclear evolution and forms a BH $\left(M_{\mathrm{BH} 1}=32.9 M_{\odot}\right)$ in a direct collapse event (no natal kick), losing $10 \%$ of its mass in neutrino emission. The mass of the $\mathrm{CO}$ core of the $\mathrm{BH}$ progenitor is $M_{\mathrm{CO}}=29.6 M_{\odot}$, which means that the first $\mathrm{BH}$ is formed with zero natal spin $\left(a_{\text {spin } 1}=0.0\right.$, see Fig. 1 for $Z=0.002$ used as representative for $Z=0.001)$.

The companion, on the other hand, has increased its mass during the mass transfer to $71.1 M_{\odot}$. It has also been rejuvenated (which we model according to Eq. (58) of Belczynski et al. $2008 \mathrm{~b}$ ) and spun up. At the offset of mass transfer, it is about $80 \%$ of its way through the MS phase. During the HG stage it expands to $1330 R_{\odot}$, which is, however, not enough to cause a Roche lobe overflow (RLOF). It further increases its radius to $1650 R_{\odot}$ as a core-He-burning $(\mathrm{CHeB})$ supergiant, at which point it initiates a dynamically unstable mass transfer and the common envelope (CE) phase. As a result, the wide binary orbit ( $a=3678 R_{\odot}$ ) decays to $a=34 R_{\odot}$, and the secondary is left without any envelope as a naked WR star. We assume a $5 \%$ Bondi-Hoyle accretion rate onto a compact object during $\mathrm{CE}$, which leads the first $\mathrm{BH}$ to only a slight increase in mass (by $\left.0.6 M_{\odot}\right)$ and spin $\left(a_{\text {spin } 1}=0.05\right)$. Shortly after $(0.3 \mathrm{Myr}$ after the CE) the secondary forms a BH with mass $M_{\mathrm{BH} 2}=24.7 M_{\odot}$ in a direct collapse with no natal kick and only $10 \%$ neutrino mass loss. Because the secondary accretes a significant fraction of its mass ( $20 \%$ ) while still on the MS, we assume that its increased rotation leads to a $\sim 20 \%$ increase of the CO core mass with respect to the non-rotating stellar models (see Appendix A.7). With the increased $\mathrm{CO}$ core mass of $M_{\mathrm{CO}}=26.0 M_{\odot}$, the second $\mathrm{BH}$ is assigned an initial spin of $a_{\text {spin2 }}=0.14$.

The BH-BH is formed after 4.9 Myr of binary evolution on a close $\left(a=37.4 R_{\odot}\right)$, almost circular $(e=0.05)$ orbit. The time to coalescence via gravitational wave emission is $6.1 \mathrm{Gyr}$. For this particular evolution/model we assume no natal kicks at the formation of the massive $\mathrm{BHs}$, so the $\mathrm{BH}$ spin vectors are aligned with the binary angular momentum $\left(\Theta_{1}=\Theta_{2}=0^{\circ}\right)$. This produces an upper limit on the effective spin parameter (see Eq. (1)). For these particular BH masses, spins and spin tilts, we obtain a rather low effective $\operatorname{spin} \chi_{\text {eff }}=0.09$. The progenitor binary forms at $z=1.2$, so $\sim 5$ Gyr after the Big Bang (close to a peak in star formation: $z \approx 2$ means $3.2 \mathrm{Gyr}$ after the Big Bang), and the $\mathrm{BH}$ BH merger takes place at $z=0.2(\sim 11$ Gyr after the Big Bang). The gravitational waves from the $\mathrm{BH}-\mathrm{BH}$ merger propagate for $\sim 2.5 \mathrm{Gyr}$ to reach the LIGO detectors at the present time. All of the system properties are within $90 \%$ of the credible limits of GW170104 (Abbott et al. 2017, see also Appendix A.5 for a revised limits on $\left.\chi_{\text {eff }}\right)$.

\section{A.5. The effective spin parameter: $\chi_{\mathrm{eff}}$}

The Bayesian analysis of GW170104 reported in Abbott et al. (2017) adopts prior assumptions about the relative likelihood of different spin magnitudes (uniform) and directions (isotropic). These assumptions are not suitable for comparison to the binary evolution model we adopt, which requires both individual spins to be initially aligned and then only mildly (if at all) misaligned by natal kicks. Recent detailed analysis addressing the impact of prior assumptions showed that they can indeed impact the inferred parameters (Vitale et al. 2017b; Williamson et al. 2017). 
We therefore reassess the reported limit, concluding that $\chi_{\text {eff }}<0.2$ at approximately $90 \%$ confidence in the context of our model. We can justify this reanalysis using only the reported LIGO result on $\chi_{\text {eff }}$, restricted to $\chi_{\text {eff }}>0$. Approximating the LIGO distribution as nearly normal with mean $\mu=-0.21$ and width $\sigma_{\chi} \simeq 0.155$, we construct a truncated normal distribution $\propto \theta(\chi) \exp -(\chi+\mu)^{2} / 2 \sigma^{2}$, which has a $90 \%$ upper limit at $\chi \simeq 0.2$.

We arrive at a similar result by reanalyzing the underlying LIGO data using the same model and techniques (including the prior), then restricting to configurations with positive individual spins $\chi_{1, z}, \chi_{2, z}>0$. Our revised upper limit is consistent with the range of plausible $\chi_{\text {eff }}$, as reported by Abbott et al. (2017) (see Fig. 5 of the supplementary material), corresponding to approximately a 98-99\% confidence limit within the strong assumptions of their original analysis.

\section{A.6. NS spins}

\section{A.6.1. Observed pulsar spin period distribution}

Although astronomers have amassed a large sample of pulsar spin-period measurements, extrapolating from observed periods to birth periods is an open area of research (for a review, see Miller \& Miller 2015). Because pulsar emission spins down the neutron star with time, any estimate of the birth period requires an estimate of the pulsar age, and an understanding of the rate of spin-down from pulsar emission (including an understanding of magnetic field evolution in neutron stars). The age of a pulsar $\left(t_{\text {pulsar }}\right)$ can be estimated assuming the angular momentum is lost through electromagnetic radiation from a pulsar with dipole magnetic fields (Manchester \& Taylor 1977):

$t_{\text {pulsar }}=\frac{P}{(n-1) \dot{P}}\left(1-\left(P_{0} / P\right)^{n-1}\right)$,

where $n$ is the pulsar braking index ( $n=3$ for magnetic dipole radiation), $P_{0}, P$ are, respectively, the initial and current pulsar spin periods. If the age of the pulsar is known by other means (e.g., the supernova remnant age), measurements of the spin period along with constraints on the braking index provide an estimate of the birth spin period. Clearly the pulsar age depends on the choice of $n$. In addition, other sources of angular momentum loss exist: for example, nonsphericities of the neutron star can cause gravitational wave emission, lowering the spin period.

Despite these uncertainties, astronomers have estimated the neutron star birth spin period distribution. The fastest pulsars could be born spinning less than $10 \mathrm{~ms}$ (the Crab pulsar is believed to have been born spinning at $17 \mathrm{~ms}$ ). Popov $\&$ Turolla (2012) found that a Gaussian with an average spin period of $100 \mathrm{~ms}$ (with a $1-\sigma$ deviation of $100 \mathrm{~ms}$ ) fits the observations. Faucher-Giguère \& Kaspi (2006) found a slightly higher average, $300 \mathrm{~ms}$ ( $1 \sigma$ deviation of $150 \mathrm{~ms})$. Igoshev \& Popov (2013) argue that the differences between these two studies could be explained by the choice of magnetic field evolution and either distribution could be made consistent with the data. Noutsos et al. (2013) also obtained a distribution of periods peaking below $125 \mathrm{~ms}$, but found an additional set of long-period birth spins $(>0.5 \mathrm{~s})$. They found that poor age estimates limit determinations of the birth pulsar spin distribution and described methods to estimate pulsar ages kinematically.

With these uncertainties in mind, we can now compare stellar models to the observed spin distribution. As with the stars forming black holes, we can estimate the birth spins of of neutron stars from their massive star progenitors by assuming that the angular momentum of the collapsing core is accreted onto the proto-neutron star along with its mass. We limit the accreted angular momentum $\left(j_{\text {acc }}\right)$ to the centrifugally supported value:

$j_{\text {acc }}=\min \left(j_{\text {shell }}, \sqrt{r_{\mathrm{NS}} G M_{\mathrm{encl}}}\right)$,

where $j_{\text {shell }}$ is the angular momentum of the accreting shell, $r_{\mathrm{NS}}$ is the neutron star radius, $G$ is the gravitational constant and $M_{\text {encl }}$ is the mass enclosed in the shell. For the neutron star radius, we assume a $10 \mathrm{~km}$. We consider only compact remnants with masses below $2.5 M_{\odot}$ as this is our adopted maximum NS mass. By summing up the angular momentum from the accreted material, we obtain the total angular momentum of the compact remnant. The moment of inertia of a neutron star $\left(I_{\mathrm{NS}}\right)$ depends upon the equation of state (Worley et al. 2008) and can vary by roughly $50 \%$ with the choice of the equation of state but it is roughly linear with neutron star mass. For our calculations, we assume:

$I_{\mathrm{NS}}=1.5 \times 10^{45}\left(M_{\mathrm{NS}} / M_{\odot}\right) \mathrm{g} \mathrm{cm}^{2}$,

where $M_{\mathrm{NS}}$ is the neutron star mass. With the total angular momentum and moment of inertia, we can determine the birth spin period of the neutron stars from our progenitors (Fig. A.3). The spins from these models assume stars born rotating at $40 \%$ breakup velocity, producing core spins near the maximum allowed by a given angular momentum transport mechanism. We have not included any angular momentum loss mechanisms, but there are several possibilities of extracting rotational energy from the protoneutron star. For example, if the rotational energy is tapped to help driving the explosion (e.g., by interaction with a disk or when forming a magnetar), the total angular momentum of the system will be reduced. Therefore the rotation rates produced in our models are only upper limits on the real spin rates.

The results presented in Fig. A.3 demonstrate that some magnetic braking is needed to reduce the angular momentum of the core, confirming decades-old arguments for magnetic breaking (Heger et al. 2000; Fryer \& Heger 2000). The Geneva models with the original distribution of angular momentum through a star produce only sub-ms pulsars. While the spins from these models without magnetic coupling have too much angular momentum, they are ideally suited to determine the amount of coupling needed to produce the correct spin periods. We assume the angular momentum is distributed with a constant angular velocity (solid-body rotation) across the mass of different cores. If we assume the angular momentum to be conserved, we can calculate the angular velocity of the core and recalculate the spin of the pulsar produced. For example, if we sum up the angular momentum of the $\mathrm{CO}$ core and divide it by its moment of inertia, we get an average angular velocity. If we use this constant angular velocity to redistribute the angular momentum in the $\mathrm{CO}$ core, we revise our estimate of the spin of the compact remnant. For this redistribution, the spin periods remain below a ms (magenta triangles). To truly slow down the birth spin periods of neutron star, we must couple the angular momentum through the helium core (meaning that one assumes a constant angular velocity from star center to the outer boundary of the helium core). Figure A.3 shows the resultant spins if we assume coupling through the helium core with a helium core definition of $X_{\mathrm{He}}>0.4, X_{\mathrm{H}}<0.01$ (blue squares), and $X_{\mathrm{He}}>0.5$ (red empty circles). This produces maximum birth spin periods of between 10-1000 ms. Further coupling, the H-rich layers, would produce spin periods (solid green circles) that are too slow to match the observed pulsar distribution. Given that these spins are maximum spin values (recall that we are using fast rotating progenitors and assuming no angular momentum loss in the explosion 


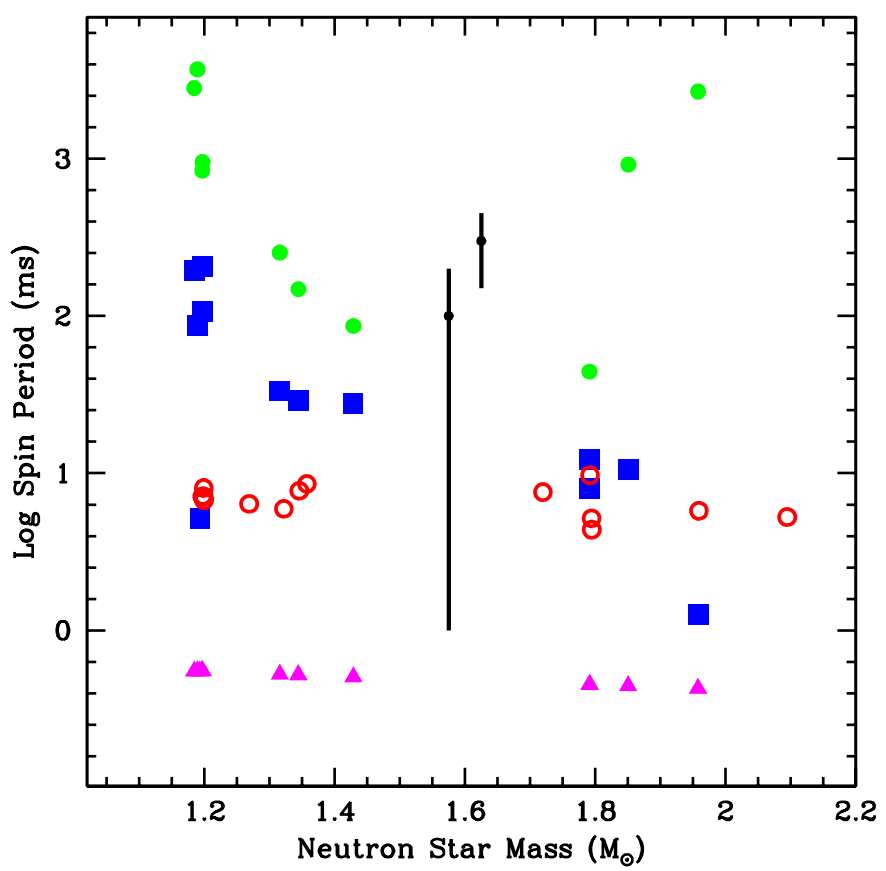

Fig. A.3. Neutron star spins from our progenitors as a function of neutron star mass. We assume any remnant from our suite of models with a mass below $2.5 M_{\odot}$ is a neutron star and consider only these remnants. With the Geneva models, we have studied the angular momentum coupling of different burning layers and their effect on the neutron star spin. The Geneva models with the original mild coupling produce spin periods shorter than $1 \mathrm{~ms}$ (not shown here, but very close to magenta triangles; see below). Some angular momentum loss in the supernova engine (e.g., magnetic coupling such as a magnetar engine) would be required to slow neutron stars down for such models to match the data. The spins are nearly the same if the coupling extends through the $\mathrm{CO}$ core (magenta triangles). Coupling through the helium layer (blue squares and empty red circles; different helium core definitions) and hydrogen layer (green solid circles) produces slower neutron stars. The heliumcoupled models match the data (but realize that we are using rapidly rotating progenitors with no angular momentum loss during NS formation). The MESA models (not shown here) with the Tayler-Spruit dynamo produce $\sim 10 \mathrm{~ms}$ pulsars; generating spins that match fastestspinning pulsars with rapidly spinning progenitor stars, an indication that the MESA models produce reasonable coupling results. The Fuller models (not shown here) do not produce rotating neutron stars, requiring spin-up mechanisms in the supernova engine to match the data. For comparison we also mark (with black lines) the range of two observational estimates. More details on models and observations are given in Appendix A.6.1.

mechanism), our pulsar spin observations argue against angularmomentum coupling beyond the helium core.

If we compare the spin periods produced using our MESA models using a prescription similar to Heger et al. (2000), we find spin periods of roughly $\sim 6-10 \mathrm{~ms}$, matching the fastestspinning non-recycled pulsars and the results of Heger et al. (2000), Fryer \& Heger (2000). These periods are produced using rapidly spinning progenitors, so we would expect their periods to match the fastest-spinning systems and this result is a relatively good match to the observed pulsar distribution. For the Fuller models, the birth spin period would be too slow to match the data, arguing for some mechanism to spin-up the neutron star. Simulations of the asymmetries in the engine do show spin-up in the core. The amount of spin-up, and whether it is sufficient to solely explain the pulsar spin periods, remains a matter of debate (Blondin \& Mezzacappa 2007; Fryer \& Young 2007; Foglizzo et al. 2009; Rantsiou et al. 2011; Kazeroni et al. 2016).

\section{A.6.2. Rotational explosion properties}

It has been argued that fast spinning magnetars can drive a subset of supernova explosions. Here we determine the role spins can play in the supernova explosion itself. With strong (magnetarstrength: $\sim 10^{15} \mathrm{G}$ ) magnetic fields, a pulsar can quickly release the rotational energy of a newly formed neutron star. To calculate the energy available for such a model, we need to estimate the rotational energy of the neutron star:

$E_{\mathrm{rot}}=1 / 2 I_{\mathrm{NS}} \omega^{2}=5 \times 10^{50}(\omega / 1000 \mathrm{~Hz})^{2} \mathrm{erg}$,

where $\omega$ is the angular velocity. If the neutron star is spinning with a ms period (e.g., original Geneva models), it can produce a $10^{51}$ erg explosion if it can tap $10 \%$ of the rotational energy to drive a jet. With the 6-10 ms periods (e.g., MESA models with rapidly rotating stars) we find that even if all of the rotational energy is tapped to drive an explosion, the rotation is unable to produce a normal-energy supernova. If the strong magnetic fields are formed quickly, a spin-powered magnetar engine will deposit its energy in the slowly-moving ejecta, accelerating this innermost material (and adding additional heating) but not contributing significantly to the total energy budget of the explosion.

Similarly, because the angular momentum is lowest in the cores of these stars, such models are unable to form a disk around the neutron star. Hence, engines that invoke jets produced by magnetic fields generated in a disk will not work. We note, however, that a disk can form after the formation of a $3 M_{\odot}$ remnant (presumably a black hole) as then more angular momentum is trapped in the remnant. It is likely that any model with Tayler-Spruit efficient angular momentum transport (e.g., MESA or Fuller models) invoking these engines will have to rely upon some means to spin up the star prior to collapse (see review by Fryer et al. 1999). We further note that this statement does not depend on the initial stellar spin of models as independent of adopted initial spin, both MESA and Fuller models end up with very small angular momentum in the core (see Fig. 23). Such rare spin-up events would be able to explain rare outbursts such as gamma-ray bursts but if these spins are correct, it is unlikely that magnetars or disks play a big role in explaining supernovae (for a review, see Fryer et al. 2019).

\section{A.7. Binary evolution calculations}

We employed the StarTrack population synthesis code (Belczynski et al. 2002, 2008b). The existing improvements relevant for massive star evolution include updates to the treatment of CE evolution (Dominik et al. 2012), the compact object masses produced by core collapse/supernovae (Fryer et al. 2012; Belczynski et al. 2012a) including the effect of pairinstability pulsation supernovae and pair-instability supernovae (Belczynski et al. 2016c), stellar binary initial conditions set by observations (de Mink \& Belczynski 2015), and observationally constrained star formation rate and metallicity evolution over cosmic time (Madau \& Dickinson 2014; Belczynski et al. 2016a). The code adopts by default the fallback-decreased natal kick prescription (see below). Additionally, we explore three different models for the $\mathrm{BH}$ natal spins proposed in the current upgrade (Sect. 2.1) as well as three different models for PPSN/PSN (Sect. 2.3).

In our population synthesis calculations we evolve stars on a finite grid of metallicity: $Z=0.0001,0.0002,0.0003,0.0004$, $0.0005,0.0006,0.0007,0.0008,0.009,0.001,0.0015,0.002$, 0.0025, 0.003, 0.0035, 0.004, 0.0045, 0.05, 0.006, 0.0065, 0.007, $0.0075,0.008,0.0085,0.009,0.0095,0.01,0.015,0.02,0.025$, 
0.03. If in our population synthesis model the metallicity is $Z<0.00089$ we adopt the BH spin model corresponding to $Z=0.0004$; if $0.00089 \leq Z<0.00346$ we adopt the BH spin model corresponding to $Z=0.002$; if $0.00346 \leq Z<0.00916$ we adopt the BH spin model corresponding to $Z=0.006$; and if $Z \geq 0.00916$ we adopt the BH spin model corresponding to $Z=0.014$. The limits are half points in decimal logarithm between the four metallicities of the $\mathrm{BH}$ natal spin model $(Z=0.014,0.006,0.002,0.0004)$

For the initial orbital period distribution of massive binaries we use $f_{\mathrm{p}}(\log p /$ day $) \propto(\log p / \text { day })^{-0.5}$ in the range of $[0.15,5.5]$ (Sana et al. 2012). For the initial eccentricity distribution we use $f_{\mathrm{e}}(e) \propto e^{-0.42}$ in the range of [0.0,0.9]. The initial mass of the primary star is taken from a broken power law IMF: $\propto M^{-1.3}$ for $0.08 \leq M<0.5 M_{\odot}, \propto M^{-2.2}$ for $0.5 \leq M<1.0 M_{\odot}$, and $\propto M^{-2.3}$ for $1.0 \leq M \leq 150 M_{\odot}$ (Kroupa 2001; Bastian et al. 2010; Duchêne \& Kraus 2013). The initial secondary mass is taken from a uniform mass ratio distribution $f_{\mathrm{q}}(q) \propto q^{0}$ in the range of $q \in[0.1,1]$.

We adopt maximum binary fraction: $f_{\mathrm{bi}}=1.0$ for stars of any mass and any metallicity. This is a sound assumption for massive stars of non-negligible metallicity (Raghavan et al. 2010; Chini et al. 2012; Moe \& Di Stefano 2013, 2017). However, the binarity may be smaller for low mass stars, for example, $f_{\mathrm{bi}}=0.5(2 / 3$ of stars in binaries). For each metallicity, we evolve $N=2 \times 10^{6}$ massive binaries (primary mass $>5 M_{\odot}$, secondary mass $>3 M_{\odot}$ ) and this corresponds to the total simulation stellar mass of $M_{\text {sim }}=1.9 \times 10^{8} M_{\odot}$ (all stars over entire IMF) for $f_{\mathrm{bi}}=1.0$. However, had we assumed $f_{\mathrm{bi}}=0.5$ for all stars, then $M_{\text {sim }}=2.8 \times 10^{8} M_{\odot}$. Therefore, such a change would decrease of all our rate predictions for double compact objects by $\sim 30 \%$ (see Eq. (7) of Belczynski et al. 2016b).

The initial distributions described above assume that all the binary parameters are independent from each other. However, various correlations between those parameters have long been suggested by the observations (e.g., Abt et al. 1990; Duchêne \& Kraus 2013). It was only recently that Moe \& Di Stefano (2017), using results from more than 20 surveys of massive binary stars, were able to fit analytic functions to the correlated distributions and obtain a join probability density function $f\left(M_{1}, q, P, e\right) \neq$ $f\left(M_{1}\right) f(q) f(P) f(e)$. In some pockets of the entire parameter space they found differences larger than an order of magnitude with respect to the typically used method of combining several independent distributions. Similarly, even though a conclusive evidence for significant IMF variations with environmental conditions is still lacking, there is an increasing amount of results suggesting such departures from the IMF universality (Bastian et al. 2010; Kroupa et al. 2013). Notably, both theoretical arguments (see Klencki et al. 2018, for an overview) and observations of some GCs in the Milky Way (Marks \& Kroupa 2012; Marks et al. 2012) seem to point towards a top-heavy $\mathrm{IMF}^{12}$ in lowmetallicity galaxies, which are likely an important formation site of massive BH-BH mergers. Recently, Klencki et al. (2018) analyzed the significance of the inter-correlations in initial distributions quantified by Moe \& Di Stefano (2017) as well as of the possible variations in the IMF slope for the massive stars on the formation rate and properties of compact binary mergers. They found that the effect of those factors is very small compared to other uncertainties (e.g., rates affected by less than a factor of 2). Their result holds even for very significant changes in the IMF slope due to the coupling of the IMF and the cosmic SFRD. This

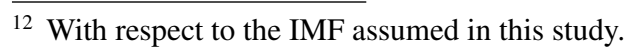

justifies the simplified assumptions of the universal IMF and non-correlated initial binary parameter distributions used in this study.

For old models (M10 and M13), we redid the calculations with the same input physics, but with the addition of the new distribution of natal BH spins. Additionally, we have updated the calibration for all models (decreasing rates by a factor of 0.926 ) to account for small inconsistencies in our previous estimates (Klencki et al. 2018). Below we comment on factors introduced in our new models (M20, M23, M25, M26).

The fraction of mass retained in the binary $\left(f_{\mathrm{a}}\right)$ during stable RLOF is not well-established, and could be fully conservative $\left(f_{\mathrm{a}}=1\right)$, fully non-conservative $\left(f_{\mathrm{a}}=0\right)$, or anywhere in between (e.g., Meurs \& van den Heuvel 1989). Donor stars are typically the more massive components, as they are the ones that evolve (and typically expand) more quickly. Consequently, more massive donors often have a much shorter thermal timescale than their companions. For that reason, in the case of a thermaltimescale mass transfer when the mass transfer rate is related to the thermal-timescale of the donor star, the less massive companions will not have enough time to thermally readjust in order to accommodate all of the transferred mass. This is likely to lead to a large fraction of the transferred mass being ejected from the system. Even in the case of a slower, nuclear-timescale mass transfer, accretion is typically expected to be small because the accretor quickly becomes spun up to its critical surface rotation velocity. We note that the picture is possibly more complicated due to the uncertain efficiency of stellar winds in carrying away the angular momentum from the accretor surface (e.g., Vanbeveren et al. 2018).

Nonetheless, we decided to revise and test our assumption on the accretion efficiency. In a previous work (as well as the remaining models in this study), we adopted $f_{\mathrm{a}}=0.5$. Recent estimates of mass transferring $\mathrm{BH}-\mathrm{BH}$ progenitors resulted (typically) in $f_{\mathrm{a}}<0.5$ (Stevenson et al. 2017), so in models M20M26 we adopt $f_{\mathrm{a}}=0.2$. As a consequence, the secondary stars (accretors) remain less massive than in our previous models, and this generates a wider $\mathrm{BH}-\mathrm{BH}$ binary mass ratio distribution than reported in our earlier studies. The efficiency of accretion in the first episode of mass transfer was noted to have possible impact on the BH-NS formation (Kruckow et al. 2018). We note that Stevenson et al. (2017) did not use a single value for $f_{\mathrm{a}}$, but, rather, estimated the accretion efficiency from the relative thermal timescales of the donor and accretor for a given binary.

Even a small amount of accretion during RLOF $(\sim$ few percent of the accretor's mass) may effectively spin up accreting stars (Packet 1981). With our adopted RLOF retention fraction of $f_{\mathrm{a}}=0.2$, accretors in $\mathrm{BH}-\mathrm{BH}$ progenitor binaries typically gain about $10 M_{\odot}$, which is enough to spin up even very massive stars. This accretion usually happens around the middle (or shortly thereafter) of the accretor's main sequence life, and therefore it allows for effective rotational mixing and the formation of more massive $\mathrm{He}$ and $\mathrm{CO}$ cores. Geneva stellar evolution models indicate that the $\mathrm{CO}$ core masses in rotating stars (40\% critical velocity) are $20 \%$ more massive than in nonrotating models. So far all $\mathrm{CO}$ core masses calculated in our binary evolution models were obtained from non-rotating models (Hurley et al. 2000). Here we increase CO core mass of accreting low-metallicity $(Z<0.002) \mathrm{MS}$ stars by $20 \%$. For high-metallicity stars, the effects of rotation on the $\mathrm{CO}$ core mass are suppressed due to angular momentum loss through stellar winds (Georgy et al. 2012). This change may increase the mass of the second $\mathrm{BH}$, and also lower its spin magnitude. In all 
models we assume that material is lost from a binary in RLOF with specific angular momentum $\mathrm{d} J / \mathrm{d} t=j_{\text {loss }}\left[J_{\text {orb }} /\left(M_{\text {don }}+\right.\right.$ $\left.\left.M_{\mathrm{acc}}\right)\right]\left(1-f_{\mathrm{a}}\right) \mathrm{d} M_{\mathrm{RLOF}} / \mathrm{d} t$, with $j_{\text {loss }}=1.0$ (Podsiadlowski et al. 1992).

Our criteria for the mass transfer stability and the occurrence of $\mathrm{CE}$ are described in detail in Sect. 5 of Belczynski et al. (2008b). To treat CE evolution, we assume energy balance with fully effective conversion of orbital energy into envelope ejection ( $\alpha=1.0)$, while the envelope binding energy for massive stars is calibrated using a parameter $\lambda$ that depends on stellar radius, mass, and metallicity for all models. For massive stars we assume $\lambda \approx 0.1 \mathrm{Xu} \& \mathrm{Li}$ (2010). Additionally, we either allow or do not allow (namely, in submodels $\mathrm{A}$ and $\mathrm{B}$ ) for $\mathrm{HG}$ stars to initiate and survive $\mathrm{CE}$ evolution Belczynski et al. (2007), Pavlovskii et al. (2017). In submodel A, only stars with well developed core-envelope boundary (beyond $\mathrm{HG}$ ) can successfully initiate and survive $\mathrm{CE}$, depending on the energy balance. In submodel B, we also allow for $\mathrm{HG}$ stars to initiate and survive CE. Recent calculations show that the accretion rates onto compact objects in $\mathrm{CE}$ inspiral can be reduced even by a factor of $10^{-2}$ with respect to the rates resulting from the Bondi-Hoyle approximation when the structure of the envelope (in particular, the density gradients around the inspiraling object) are taken into account (Ricker \& Taam 2008; MacLeod et al. 2017; Murguia-Berthier et al. 2017; Holgado et al. 2018). MacLeod \& Ramirez-Ruiz (2015) argue that accretion structures forming around compact objects embedded in the CE may span a large fraction of the envelope radius, and so traverse substantial density gradients. Introducing gradients in the CE structure leads to net non-zero angular momentum of the flow around an accreting object (which is not the case in the standard Hoyle formalism) and in doing, it limits accretion: steeper density gradients correspond to smaller accretion. The typical values of the density gradients found by these authors introduce a considerable perturbation to the flow. For most density gradients considered by MacLeod et al. (2017), the accretion rate is well below $10 \%$ of Bondi-Hoyle accretion rate. Based on these findings, we adopt $f_{\text {bond }}=5 \%$ of the Bondi-Hoyle accretion rate onto a $\mathrm{BH}$ in $\mathrm{CE}$ in our current simulations. Therefore, massive BHs $\left(M_{\mathrm{BH}} \sim 30 M_{\odot}\right)$ accrete $\sim 0.5 M_{\odot}$ in a typical CE event, as opposed to $\sim 1.0 M_{\odot}$ in our earlier calculations $\left(f_{\text {bond }}=10 \%\right)$. To assess the Bondi-Hoyle accretion rate we follow the approach presented in Belczynski et al. (2002).

The spectroscopic analysis of OB stars in the Local Group (MW, SMC, LMC, e.g., Oskinova et al. 2013; Hainich et al. 2018; Ramachandran et al. 2019) as well as other low-metallicity dwarf galaxies (e.g., Bouret et al. 2015) has shown a systematic offset in the wind mass loss rates between theoretical predictions (Vink et al. 2001, assumed in most our models) and the empirical $\log \dot{M}-\log L$ relation. Namely, the theoretical models seem to overestimate the actual wind mass loss rates of hot stars by at least a factor of a few. The most likely cause for this discrepancy is clumping of the wind, which is not accounted for in the standard models of radiatively driven smooth winds. For that reason, in model M50, we reduce all the wind mass loss rates down to $30 \%$ of the usually assumed values.

Compact remnants formed in supernovae can receive proper motions via two classes of engine-driven natal kicks: asymmetric matter ejecta or asymmetric neutrino emission. For BHs, asymmetric matter ejection mechanisms only work when matter is ejected, as opposed to prompt collapse or complete recapture of all ejected material ("fallback"). In our calculations, we expect that only a small fraction of systems eject a substantial amount of matter, enabling a substantial $\mathrm{BH}$ natal recoil kick. In contrast, asymmetric neutrino emission mechanisms operate even without any mass ejection, and thus they can affect any model of $\mathrm{BH}$ formation. Although neutrino mechanisms have been invoked to explain recoil velocities of pulsars and X-ray binaries (Lai \& Qian 1998; Repetto \& Nelemans 2015), the proposed kick models all require strong magnetic fields. Models without strong magnetic fields are unable to produce significant neutrino kicks (Tamborra et al. 2014).

The sterile neutrino oscillation model (Kusenko \& Segrè 1996; Fryer \& Kusenko 2006) argues that neutrinos produced in the core could oscillate to sterile neutrinos and escape the core. Large magnetic fields align the ions and electrons, forcing both the neutrino scattering and absorption cross sections to be anisotropic. To ensure asymmetric neutrino emission, these strong magnetic fields must be at the last scattering surface for the neutrinos. If the magnetic field in the core is high enough to align the ions and electrons, the neutrinos in the core will be anisotropic. If these neutrinos oscillate into sterile neutrinos, they can escape, retaining their anisotropies and generating large natal kicks.

Alternatively, the neutrino bubble instability (Socrates et al. 2005) argues that magnetic-acoustic instabilities develop, transporting neutrino radiation to the photosphere. These instabilities carry neutrinos, and the luminosity escaping the neutrinosphere will be enhanced at these "bubbles". If the magnetic-acoustic bubbles are globally asymmetric, the neutrino emission will also be asymmetric, producing a neutrino-driven kick. Current supernova calculations have several limitations: (i) they do not model high magnetic fields; (ii) they do not sufficiently resolve the hydrodynamics; and (iii) they do not include the neutrino oscillation physics necessary to produce these kicks. So high, neutrinodriven $\mathrm{BH}$ natal kicks cannot be ruled out.

In models M10 and M20, we test asymmetric mass ejection kicks, as we employ fallback-decreased natal kicks Fryer et al. (2012). To mimic asymmetric neutrino emission mechanisms, we explore an alternative phenomenological prescription for $\mathrm{BH}$ natal kicks in models M13, M23, M25, M26, M33, M35, and M43, where we impart kicks which are random in direction, with magnitude drawn from a Maxwellian with a given 1-dimensional $\sigma$, independent of the $\mathrm{BH}$ mass or its progenitor history (see Table 2).

\section{A.8. Detectabilty of mergers in gravitational waves}

All the compact object mergers are redistributed according to star formation history across cosmic time $(z \approx 0-15$ for Population I and II stars; e.g., Madau \& Dickinson 2014), taking into account the time delay between binary formation at Zero Age Main Sequence and the merger. For each merger at redshift $z$, we use phenomenological inspiral-merger-ringdown waveforms (IMRPhenomD; Khan et al. 2016) to calculate the signal-to-noise ratio in the $\mathrm{O} 1 / \mathrm{O} 2 \mathrm{LIGO}$ runs. A given merger is considered detectable (depending on its random sky location and orbital orientation with respect to the detectors) if the signal-to-noise ratio $(\mathrm{S} / \mathrm{N})$ in a single detector is greater than 8 . Only detectable mergers are used in our comparisons with $\mathrm{O} 1 / \mathrm{O} 2$ data (e.g., rates in Table 3 or effective spins in Sect. 3.4).

With this method, we obtain a self-consistent redshift distribution of mergers in the local Universe, and we also account for LIGO/Virgo detectability of our synthetic mergers (in particular, more massive mergers can be detected at larger redshifts). A more detailed description of detectability criteria can be found in Belczynski et al. (2016b,a,c). 


\section{A.9. BH-NS merger rate limits from the ongoing $\mathrm{O} 3$ run}

Here, we analyze what the constraint on the rate density of $\mathrm{BH}$ NS mergers that the recent LIGO/Virgo candidate (LVC 2019a,b, S190814bv) ${ }^{13}$ entails.

We do not know the mass of the object, but we assume that the mass of the NS $M_{\mathrm{NS}}$ must be in the range from 1.3 to $3 M_{\odot}$, while the $\mathrm{BH}$ mass $M_{\mathrm{BH}}$ is in the range from 5 to $50 M_{\odot}$. We denote the chirp mass of the system as $\mathcal{M}$. The O3 has lasted for about 4.5 months with the $80 \%$ uptime which given the effective observation time $t_{\mathrm{obs}}$ of 150 days, or 0.41 years for $\mathrm{O} 1 / \mathrm{O} 2$, and $t_{\mathrm{obs}}$ of 3.6 months, or 0.30 years. For the range of the search, we assume that the sensitivity to NS-NS mergers was $r_{\mathrm{NSNS}}=80 \mathrm{Mpc}(\mathrm{O} 1 / \mathrm{O} 2)$ and $r_{\mathrm{NSNS}}=135 \mathrm{Mpc}(\mathrm{O} 3)$. We can estimate the range of $\mathrm{BH}-\mathrm{NS}$ merges as:

$r_{\mathrm{BHNS}}=r_{\mathrm{NSNS}} \times\left(\mathcal{M} / 1.2 M_{\odot}\right)^{5 / 6}$,

where we assume that the fiducial chirp mass of a NS-NS system is $1.2 M_{\odot}$. The observed volume is then $V_{\mathrm{BHNS}}=(4 / 3) \pi r_{\mathrm{BHNS}}^{3}$.
The rate density of BH-NS mergers, estimated from this one event, can be estimated as

$$
\mathcal{R}=\frac{1}{\left(t_{\mathrm{obs}} V_{\mathrm{BHNS}}\right)_{\mathrm{O} 1 / \mathrm{O} 2}+\left(t_{\mathrm{obs}} V_{\mathrm{BHNS}}\right)_{\mathrm{O} 3}}=1.6-60 \mathrm{Gpc}^{-3} \mathrm{yr}^{-1},
$$

and the range corresponds to the limits at which we have allowed NS and BH mass to vary. This estimate will go down by approximately a factor of two if no similar objects are further detected in the remainder of $\mathrm{O} 3(0.7 \mathrm{yr})$. On the other hand, the range becomes narrower once we know the mass estimates of NS and $\mathrm{BH}$. We note however, that including the effect of Poisson distribution width will broaden the result again. Thus we are confident that the above estimate is close to accurate under the assumption that the detected system was, in fact, a BH-NS merger and not a $\mathrm{BH}-\mathrm{BH}$ merger with a very light secondary $\mathrm{BH}$. We confront the merger rate limit estimated above with the results from our models in Fig. 25.

\footnotetext{
${ }^{13}$ See https://gracedb.ligo.org/superevents/S190814bv/
} 